\title{
Modelagem conjunta de dados longitudinais contínuos no intervalo unitário e tempos de sobrevivência
}

\author{
Aline Campos Reis de Souza
}

TESE APRESENTADA

AO

INSTITUTO DE MATEMÁTICA E EsTATÍSTICA

DA

Universidade DE SÃo PaUlo

PARA

OBTENÇÃO DO TÍTULO

$\mathrm{DE}$

DOUTOR EM CIÊNCIAS

Programa: Estatística

Orientador: Prof. Dr. Antonio Carlos Pedroso de Lima

Durante o desenvolvimento deste trabalho o autor recebeu auxílio financeiro da CAPES e da CNPq 


\section{Modelagem conjunta de dados longitudinais contínuos no intervalo unitário e tempos de sobrevivência}




\section{Agradecimentos}

Ao longo do desenvolvimento desta tese diversas situações me colocaram na condição aprendiz, me convidando a lidar com o mundo e suas adversidades dentro e fora do ambiente acadêmico. Esse processo de aprendizado, embora intenso e extremamente desafiador, nunca foi solitário. A sua conclusão é um somatório de pequenas superações, alcançadas com o suporte de algumas pessoas para as quais dedico as seguintes palavras de agradecimento.

Começo pelo meu orientador Antonio Carlos Pedroso de Lima, com quem tive a sorte de desenvolver este trabalho durante o período de doutorado. Nos últimos anos sempre fui estimulada a discutir sobre a tese, me sentindo a vontade para expor novas ideias, recorrer às suas sugestões ou mesmo reclamar das tentativas frustradas de resolver os problemas que surgiam. Nos momentos que mais precisei sua orientação transcendeu a Estatística em conversas repletas de cuidado e paciência, me ajudando a lidar com diversas nuances da vida. Esta experiência me enriqueceu como pessoa e me tornou melhor em diferentes aspectos. Encerro esta etapa com muita gratidão e admiração, além de boas lembranças das reuniões presenciais e remotas via Google Meet.

Agradeço ao professor Vicente G. Cancho, que foi meu orientador e um grande incentivador durante o mestrado no ICMC-USP, me proporcionando oportunidades pelas quais sou extremamente grata. Essa motivação foi um importante combustível no doutorado nos momentos em que a pesquisa parecia ser impossível de ser executada. Obrigada por acreditar no meu trabalho e por me inspirar a enfrentar os problemas com muita persistência, rigor e criatividade.

Aos colegas que conheci no doutorado do IME-USP, em especial à Awo Sitso, Hellen, Jhenifer, Simone e Tuany - o meu muito obrigada! Nesse período foram incontáveis as vezes que nos apoiamos, nos divertimos e fofocamos no café, laboratório, no apartamento da Sitso ou mesmo à distância, via WhatsApp. Acumulamos boas estórias que adoramos relembrar. E com uma boa dose de otimismo, ainda teremos muitas outras novas para vivenciar. Aos amigos Brian, Carlos e Dani do DES-UEM, agradeço por fazerem parte deste processo, que envolveu sugestões, flexibilização da carga horária para que as minhas viagens à São Paulo fossem possíveis e diversos momentos agradáveis de confraternização. À Terezinha Késsia (TT), 
agradeço pela participação ativa nesta tese. Nos últimos anos fomos colegas de doutorado e de trabalho. Dividimos sala, casa, conquistas, aflições, confidências... E crescemos juntas em todas estas situações. Obrigada por tornar meus dias mais alegres com seus áudios e vídeos compartilhados. E por me emprestar o caderno de Estatística Avançada do professor Carlos Alberto (que ainda busco sem sucesso pelo IME-USP).

Agradeço ao meu marido Willian por me apresentar a Estatística e torná-la uma opção viável em minha vida. Desde o início sempre tive a sua disponibilidade para ouvir cada detalhe deste trabalho, a qualquer horário, com muita atenção e paciência. Conviver com você sempre foi algo divertido e agradável, e enfrentar este desafio foi muito mais fácil com o seu apoio, carinho e companheirismo.

Aos meus pais Edson e Regina, todo meu amor e gratidão pelos ensinamentos que me formaram como pessoa. Também agradeço ao meu tio e grande amigo Milton por me receber em São Paulo (pela segunda vez) e por proporcionar longas conversas à uma pessoa que adora ouvir sobre as suas aventuras malucas. Aos meus irmãos Thais e Matheus, o carinho de vocês foi essencial para a conclusão desta etapa.

Agradeço aos professores, funcionários e amigos do Instituto de Ciências Matemáticas e de Computação da USP que contribuíram com a minha formação e participaram de importantes momentos da minha vida e ao Instituto de Matemática e Estatística por toda estrutura oferecida para o desenvolvimento deste trabalho. Deixo um agradecimento especial à Regiane Guimarães pela sua boa vontade em resolver diversas questões burocráticas ao longo do doutorado.

Finalmente, agradeço à CAPES e ao $C N P q$ pelo apoio financeiro para a execução desta pesquisa e para a participação em eventos científicos. 


\section{Resumo}

SOUZA, A. C. R. Modelagem conjunta de dados longitudinais contínuos no intervalo unitário e tempos de sobrevivência. 2021. 127 f. Tese (Doutorado) - Instituto de Matemática e Estatística, Universidade de São Paulo, São Paulo, 2021.

Em diversas pesquisas os benefícios do tratamento intensivo em pacientes com câncer são investigados por meio de estudos longitudinais, nos quais medições repetidas de escores de qualidade de vida são observadas juntamente com os tempos até a ocorrência do óbito ou de algum evento clínico de interesse. Nesses cenários, indivíduos com piores condições de saúde são mais propícios ao abandono do estudo devido a morte ou abdicação do tratamento. A modelagem conjunta dos processos longitudinal e de sobrevivência possibilita a investigação simultânea dessas respostas e permite quantificar a associação existente entre as mesmas. Motivado por um estudo longitudinal envolvendo pacientes oncológicos que deram entrada em unidades de terapia intensiva, este trabalho contribui com a modelagem conjunta de componentes longitudinais restritas ao intervalo unitário e tempos de sobrevivência com o objetivo de (i) estimar o impacto da evolução das trajetórias longitudinais de qualidade de vida na taxa de mortalidade e (ii) avaliar o potencial preditivo dos escores de qualidade de vida na estimação das sobrevidas individuais. As vantagens da abordagem desenvolvida são acessadas através de um estudo de simulação, que verifica os efeitos da má especificação da componente longitudinal. Além disso, do ponto de vista computacional, um conjunto de reparametrizações da função de verossimilhança é proposta através de técnicas de precondicionamento, visando reduzir o alto custo da estimação de máxima verossimilhança.

Palavras-chave: distribuição beta, distribuição beta inflacionada em um, modelos conjuntos, precondicionamento, qualidade de vida relacionada à saúde. 


\section{Abstract}

SOUZA, A. C. R. Joint modeling of continuous longitudinal data in the unit interval and survival times. 2021. 107 f. Tese (Doutorado) - Instituto de Matemática e Estatística, Universidade de São Paulo, São Paulo, 2021.

In several studies, the benefits of intensive care in cancer patients are investigated through longitudinal studies, which include repeated quality of life scores that are observed along with the times until the occurrence of death or of some clinical event of interest. In these scenarios, individuals with worse health conditions are more likely to drop out of the study, due to death or abdication of treatment. The joint modeling of longitudinal processes and survival times enables the simultaneous investigation of the responses, in addition to defining an existing association between them. Motivated by a longitudinal study involving cancer patients admitted to intensive care units, this work contributes with an appropriate modeling of longitudinal components restricted to the unit interval and survival times, to (i) estimate the association between the evolution of measures of quality of life and survival process, and (ii) assess the predictive potential of quality of life scores in estimating individual survival. The advantages of the developed approaches are accessed through a simulation study, with the objective of verifying the effects of misspecification of the longitudinal component. Furthermore, from a computational point of view, a set of reparametrizations of the likelihood is proposed through preconditioning techniques, simplifying the high cost of maximum likelihood estimation.

Keywords: beta distribution, heath related quality of life, joint models, one inflated beta distribution, preconditioning. 


\section{Sumário}

Lista de Figuras

Lista de Tabelas $\quad$ x

1 Introdução 1

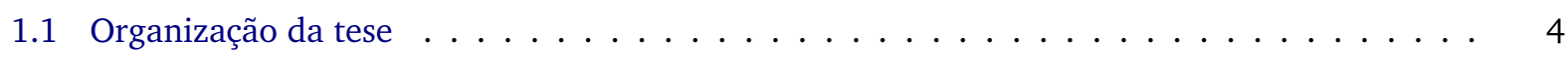

2 Motivação $\quad 5$

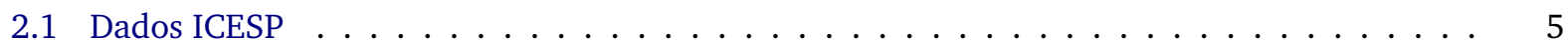

2.1 .1 Escores de qualidade de vida $\ldots \ldots \ldots \ldots \ldots \ldots$

2.2 Análise exploratória das respostas longitudinais $\ldots \ldots \ldots \ldots \ldots \ldots$

2.3 Escores de qualidade de vida como preditores da mortalidade . . . . . . . . . . . . . . . 12

3 Modelos conjuntos para dados longitudinais e tempos de sobrevivência 15

3.1 Motivação para a modelagem conjunta . . . . . . . . . . . . . . . . . 15

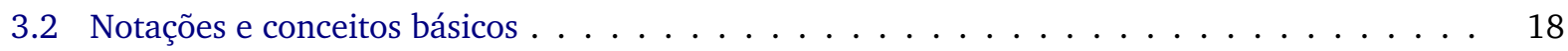

3.3 Modelagem da componente longitudinal . . . . . . . . . . . . . . . . . . . . . . . . 19

3.4 Modelagem da componente de sobrevivência $\ldots \ldots \ldots \ldots$

3.5 Estruturas de ligação . . . . . . . . . . . . . . . . . . . . . . . . 20

3.6 Estimação . . . . . . . . . . . . . . . . . . . . . . . . 22

3.7 Predições das probabilidades de sobrevivência $\ldots \ldots \ldots \ldots \ldots \ldots \ldots \ldots \ldots$

4 Modelos conjuntos para dados longitudinais contínuos no intervalo unitário e tempos de sobrevivência $\quad 25$

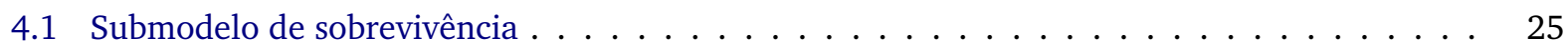

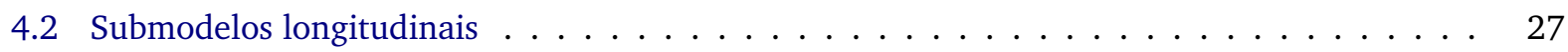

4.2.1 Modelo de regressão beta com efeitos aleatórios . . . . . . . . . . . . . . . . . . 27

4.2.2 Modelo de regressão beta inflacionado em um com efeitos aleatórios . . . . . . . . 28 
4.2.3 Objetivos inferenciais da modelagem proposta . . . . . . . . . . . . . . . . . 29

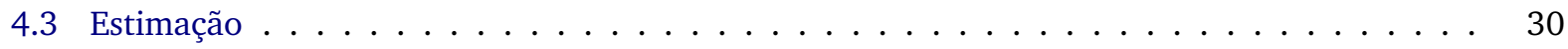

4.4 Predições de qualidade de vida e de sobrevivência . . . . . . . . . . . . . . . . . . . 32

4.5 Erros padrão bootstrap para as predições de sobrevivência $\ldots \ldots \ldots$. . . . . . . . . . 34

4.5.1 Boostrap balanceado para dados longitudinais e de sobrevivência $\ldots \ldots \ldots$. . . . 35

5 Aspectos computacionais do processo de estimação dos modelos conjuntos 38

5.1 Métodos iterativos e ordem de convergência . . . . . . . . . . . . . . 41

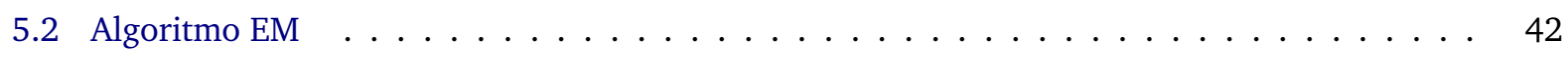

5.3 Extensões do algoritmo EM $\ldots \ldots \ldots \ldots \ldots \ldots \ldots \ldots \ldots$

5.4 Métodos de busca linear . . . . . . . . . . . . . . . . . 46

5.4 .1 Algoritmos de busca linear . . . . . . . . . . . . . . . . 47

6 Técnicas de precondicionamento para a estimação de modelos conjuntos $\quad 50$

6.1 Condicionamento de sistemas lineares . . . . . . . . . . . . . . . 51

6.1 .1 Número de condição de um sistema linear . . . . . . . . . . . . . . . . . 52

6.2 Condicionamento em um problema de maximização $\ldots \ldots \ldots \ldots$. . . . . . . 53

6.3 Técnicas de precondicionamento para a estimação de modelos conjuntos . . . . . . . . . . 55

6.3.1 Reescala baseada em valores típicos das variáveis . . . . . . . . . . . . . . 57

6.3.2 Reescala baseada no precondicionador de Jacobi . . . . . . . . . . . . . . . 58

7 Estudo de simulação $\quad 59$

7.1 Geração dos dados . . . . . . . . . . . . . . . . . . . . . . . . 59

7.1.1 Geração conjunta de dados longitudinais e tempos de sobrevivência . . . . . . . . 61

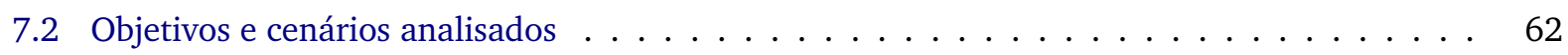

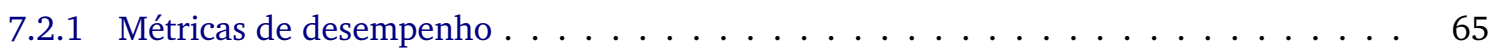

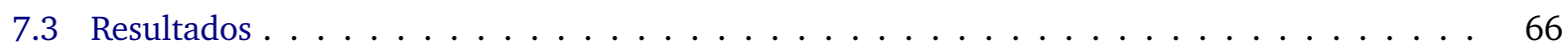

7.3.1 Desempenho dos estimadores de máxima verossimilhança . . . . . . . . . . 66

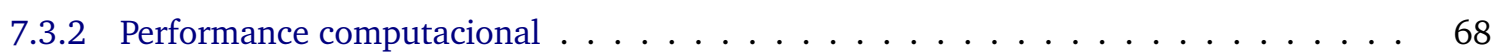

8 Análise dos dados do ICESP $\quad 77$

8.1 Especificação dos modelos conjuntos $\ldots \ldots \ldots \ldots \ldots \ldots \ldots \ldots$

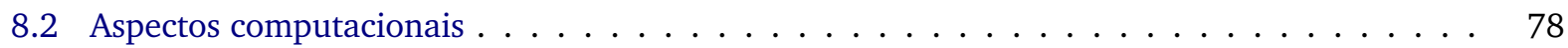

8.3 Estimação . . . . . . . . . . . . . . . . . . . . . . 80

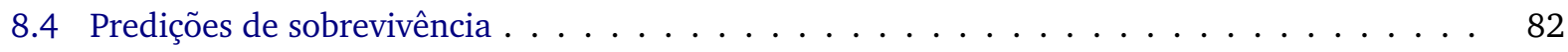

8.4.1 Performance dos índices de utilidade como preditores de mortalidade . . . . . . . . 83 
8.4.2 Estudo de casos com predições de sobrevidas individuais . . . . . . . . . . . . . . 84

9 Discussão e propostas $\quad 95$

$\begin{array}{ll}\text { A Conceitos gerais de análise numérica } & 97\end{array}$

A.1 Regras de quadratura para integração numérica . . . . . . . . . . . . . . . . . 97

A.2 Método das diferenças finitas $\ldots \ldots \ldots \ldots \ldots \ldots \ldots \ldots$

$\begin{array}{ll}\text { B Variáveis basais do estudo do ICESP } & 100\end{array}$

$\begin{array}{ll}\text { C Códigos } & 101\end{array}$

C.1 Estruturas de dados usadas para a vetorização no R . . . . . . . . . . . . . . . . . . . . 101

C.2 Verossimilhança marginal do modelo BE-WEI _ . . . . . . . . . . . . . . . . . 103

C.3 Reescala dos parâmetros através do optim . . . . . . . . . . . . . . . . . . 104

$\begin{array}{ll}\text { Referências Bibliográficas } & 105\end{array}$ 


\section{Lista de Figuras}

2.1 Boxplots e histogramas de frequência para as observações dos índices de utilidade, considerando: à esquerda, os dados em sua escala original (IU); ao centro, a transformação de Smithson e Verkuilen (2006) das medidas $(I U(0,1))$; e à direita, a transformação logito dos valores observados $($ logito $I U) \ldots \ldots \ldots \ldots \ldots \ldots \ldots \ldots \ldots \ldots \ldots \ldots \ldots \ldots$

2.2 Boxplots e histogramas de frequência para as observações dos IUs para os diferentes períodos do acompanhamento longitudinal. . . . . . . . . . . . . . . . . . . .

2.3 À esquerda, o mapa de calor para os IUs médios de pacientes estratificados segundo os tempos da última medição longitudinal $(t)$, em cada período de coleta dos dados. À direita, a evolução da proporção de pacientes no estudo no período do seguimento longitudinal. . . . 11

2.4 Estimativa de Kaplan-Meier para a probabilidade de sobrevivência dos 803 pacientes do estudo. 12

2.5 Observações longitudinais dos índices de utilidade (pontos abertos) e trajetórias individuais médias (retas azuis), obtidas através do método LOESS, para 8 indivíduos selecionados aleatoriamente na amostra do ICESP. . . . . . . . . . . . . .

7.1 Fluxograma com as técnicas para a simulação dos tempos de sobrevivência de um modelo de riscos proporcionais abordadas em Crowther e Lambert (2013) . . . . . . . . . . . . . . . .

7.2 Boxplots com as medidas de desempenho computacional da estimação de máxima verossimilhança do modelo BEI-WEI considerando o seu ajuste às 500 réplicas dos dados gerados pelo modelo BEI-WEI e utilizando as técnicas de precondicionamento propostas, . . . . . . . . . 75

7.3 Boxplots com as medidas de desempenho computacional da estimação de máxima verossimilhança do modelo BE-WEI considerando o seu ajuste às 500 réplicas dos dados gerados pelo modelo BEI-WEI e utilizando as técnicas de precondicionamento propostas, $\ldots \ldots \ldots \ldots \ldots \ldots$

8.1 Estimativas para os erros de predição das probabilidades de sobrevivência condicionais $\hat{\pi}(u \mid t)$, com $t=15,90,180,360,540)$, obtidas através de 20 repetições do procedimento de validação cruzada. . . . . . . . . . . . . . . . . . . . . . . . . . . 
8.2 Índices de utilidade (IUs) observados e predições de sobrevivência para pacientes do estudo do ICESP, segundo os modelos conjuntos BE-WEI e BEI-WEI e LN-WEI. . . . . . . . . . . 89

8.3 Índices de utilidade (IUs) observados e predições das sobrevidas condicionais para o paciente 441, segundo os modelos conjuntos BE-WEI e BEI-WEI e LN-WEI. . . . . . . . . . . . . . 90

8.4 Índices de utilidade (IUs) observados e predições das sobrevidas condicionais para o paciente 37, segundo os modelos conjuntos BE-WEI e BEI-WEI e LN-WEI. . . . . . . . . . . . . . .

8.5 Índices de utilidade (IUs) observados e predições das sobrevidas condicionais para o paciente 165, segundo os modelos conjuntos BE-WEI e BEI-WEI e LN-WEI . . . . . . . . . . . . . . 


\section{Lista de Tabelas}

2.1 Número de indíviduos na amostra, médias e desvios padrão dos $I U$ 's observados, e frequência relativa de IU's máximos $(\%)$ para cada instante de coleta. . . . . . . . . . . . . . . 10

7.1 Ilustração das observações de uma unidade amostral de um conjunto de dados genérico, resultante do processo de simulação descrito em Crowther e Lambert (2013) para a geração de dados provenientes de um modelo conjunto. . . . . . . . . . . . . . . . . .

7.2 Valores dos parâmetros utilizados na geração conjunta dados longitudinais e de sobrevivência.

7.3 Medidas das estimativas dos parâmetros dos modelos BEI-WEI, BE-WEI e LN-WEI: viés (VI), viés relativo (VIR), raiz do erro quadrático médio (REQM) e raiz do erro quadrático médio relativo (REQMR), para dados gerados do modelo BEI-WEI com N $=500$, Pc $=0 \% \mathrm{e}$

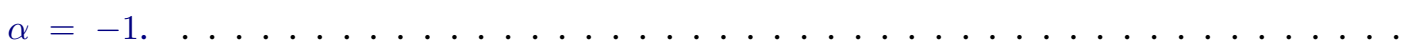

7.4 Medidas das estimativas dos parâmetros dos modelos BEI-WEI, BE-WEI e LN-WEI: viés (VI), viés relativo (VIR), raiz do erro quadrático médio (REQM) e raiz do erro quadrático médio relativo (REQMR), para dados gerados do modelo BEI-WEI com N $=500$, Pc $=20 \% \mathrm{e}$

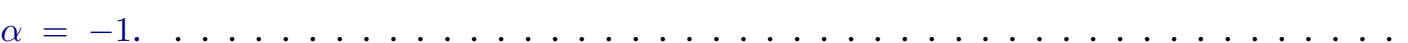

7.5 Medidas das estimativas dos parâmetros dos modelos BEI-WEI, BE-WEI e LN-WEI: viés (VI), viés relativo (VIR), raiz do erro quadrático médio (REQM) e raiz do erro quadrático médio relativo (REQMR), para dados gerados do modelo BEI-WEI com $\mathrm{N}=500$, Pc $=0 \% \mathrm{e}$ $\alpha=-4$

7.6 Medidas das estimativas dos parâmetros dos modelos BEI-WEI, BE-WEI e LN-WEI: viés (VI), viés relativo (VIR), raiz do erro quadrático médio (REQM) e raiz do erro quadrático médio relativo (REQMR), para dados gerados do modelo BEI-WEI com $\mathrm{N}=500, \mathrm{Pc}=20 \% \mathrm{e}$ $\alpha=-4$. 
7.7 Percentuais de estimativas de associações significativas obtidas através do ajuste dos modelos BEI-WEI, BE-WEI e LN-WEI nas 500 réplicas geradas a partir do modelo BEI-WEI, considerando diferentes graus de dependência $(\alpha)$ entres as respostas simuladas e percentuais de observações unitárias na amostra $\left(P_{\max }\right)$ iguais a $0,5 \%, 10 \%$ e $20 \%$. . . . . . . .

7.8 Percentuais de estimativas de associações significativas obtidas através do ajuste dos modelos BEI-WEI, BE-WEI e LN-WEI nas 500 réplicas geradas a partir do modelo BE-WEI, considerando diferentes graus de dependência $(\alpha)$ entres as respostas simuladas e percentuais de observações unitárias na amostra $\left(P_{\max }\right)$ iguais a $0,5 \%, 10 \%$ e $20 \% \ldots \ldots \ldots \ldots$

8.1 Desempenho do processo de estimação dos modelos conjuntos, considerando a reescala da verossimilhança através dos fatores determinados pelo vetor identidade, por valores típicos dos parâmetros e pelo precondicionador de Jacobi. . . . . . . . . . . . . . . . .

8.2 Desempenho do processo de estimação do modelo conjunto LN-WEI no pacote JM, considerando a reescala da verossimilhança através dos fatores determinados pelo vetor identidade, por valores típicos dos parâmetros e pelo precondicionador de Jacobi. . . . . . . . . . . . . .

8.3 Estimativas para o processo longitudinal, considerando os modelos BE-WEI, BEI-WEI e

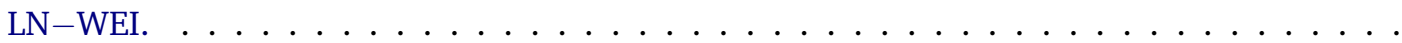

8.4 Estimativas para o processo de sobrevivência, considerando os modelos BE-WEI, BEI-WEI

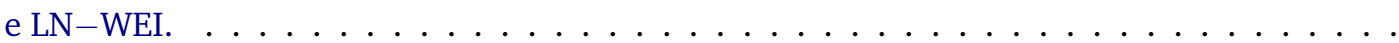

8.5 Perfil dos pacientes selecionados segundo as variáveis explicativas radioterapia prévia, delirium, ECOG, extensão do câncer e tipo admissão UTI; e respostas de sobrevivência observadas. 86

8.6 Predições de sobrevivência condicionais para os pacientes 441,37 e 165 após a coleta da medição $y_{180}$, em $u=t+\Delta t$, com $\Delta t=30,60,90$ e $180 \ldots \ldots \ldots \ldots \ldots$

8.7 Predições de sobrevivência condicionais do paciente 441 após a inclusão da medida longitudinal $y_{t}(t=15,90,180,360,540)$ e estimativas bootstrap para o seus respectivos erros padrão $\left(\widehat{E P}_{B}\right)$ em $u=t+\Delta t$, com $\Delta t=30,60,90$ e $180 \ldots \ldots \ldots \ldots \ldots$

8.8 Predições de sobrevivência condicionais do paciente $\mathbf{3 7}$ após a inclusão da medida longitudinal $y_{t}(t=15,90,180)$ e estimativas bootstrap para o seus respectivos erros padrão $\left(\widehat{E P}_{B}\right)$ em $u=t+\Delta t$, com $\Delta t=30,60,90$ e $180 \ldots \ldots \ldots \ldots \ldots \ldots \ldots$

8.9 Predições de sobrevivência condicionais do paciente 165 após a inclusão da medida longitudinal $y_{t}(t=15,90,180,360,540)$ e estimativas bootstrap para o seus respectivos erros padrão $\left(\widehat{E P}_{B}\right)$ em $u=t+\Delta t$, com $\Delta t=30,60,90$ e $180 \ldots \ldots \ldots \ldots \ldots$ 


\section{Capítulo 1}

\section{Introdução}

Nas últimas décadas as medidas de qualidade de vida relacionada à saúde têm se consolidado como um importante desfecho em diversas pesquisas clínicas, especialmente em estudos sobre o tratamento do câncer, nos quais melhorias modestas na sobrevida são ponderadas pelos benefícios gerados ao bemestar dos pacientes. Esse é o caso, por exemplo, dos ensaios clínicos em medicina intensiva que consideram não somente a mortalidade no contexto dos cuidados intensivos, como também as implicações decorrentes de tais intervenções (Vesz et al., 2013; Shrestha et al., 2019).

Os efeitos de um tratamento na qualidade de vida de uma população de interesse podem ser investigados por meio de estudos longitudinais, que permitem analisar entre outros fatores, a evolução dos estados de saúde de cada paciente ao longo do tempo. Indivíduos gravemente enfermos, e consequentemente com baixos escores de qualidade de vida, estão mais propícios às perdas de acompanhamento devido ao abandono ou ao óbito. Nesses cenários, grande parte das evasões podem ser informativas sendo importante incorporar este aspecto na modelagem dos dados.

De modo geral, em aplicações que envolvem medidas longitudinais de qualidade de vida e tempos até a ocorrência de um evento de interesse recomenda-se que as respostas sejam analisadas de forma conjunta. Segundo Billingham e Abrams (2002), essa prática pode oferecer uma série de vantagens como (i) o aprimoramento dos resultados inferenciais, geralmente obtidos por meio da modelagem independente de cada uma das variáveis; (ii) a acomodação dos abandonos informativos ocasionados pelos óbitos dos pacientes ao longo do acompanhamento; e (iii) a extensão da análise aos abandonos informativos observados em períodos que antecedem o óbito (observações censuradas). 
Entre os diferentes tipos de abordagens conjuntas empregadas a esses tipos de dados citam-se: os anos de vida ajustados para a qualidade de vida (QALYs), que consiste na combinação das respostas por meio de uma única medida quantitativa; os modelos multiestados, que consideram a modelagem das transições entre os estados de saúde dos pacientes; e os modelos conjuntos para dados longitudinais e tempos até a ocorrência de um evento de interesse (Tsiatis e Davidian, 2004; Rizopoulos, 2012), compostos por dois submodelos que descrevem o processo longitudinal e de sobrevivência, e por uma estrutura de ligação que induz a associação entre estas respostas através do compartilhamento de um conjunto de variáveis não observáveis (efeitos aleatórios).

No âmbito dos modelos conjuntos, ao contrário das metodologias previamente mencionadas, é necessário considerar pressupostos distribucionais a respeito das medidas repetidas. No caso específico em que as variáveis longitudinais são contínuas e restritas a um intervalo, é comum assumir normalidade mediante o uso de transformações dos dados. Entretanto, conforme argumentado em Ferrari e CribariNeto (2004), Hunger et al. (2011) e Galvis et al. (2014), esta estratégia não leva em conta características importantes como assimetria e heteroscedasticidade, tipicamente observadas em respostas limitadas.

Na abordagem conjunta, a descrição desses aspectos pode ser feita através da modelagem direta das componentes longitudinais. Nesta direção, Song et al. (2017) apresentam uma proposta para a sua especificação com base na distribuição simplex (Barndorff-Nielsen e Jørgensen, 1991), visando comparar os efeitos de dois tratamentos quimioterápicos na evolução da qualidade de vida de pacientes oncológicos. Em Hatfield et al. (2012), uma análise bayesiana foi conduzida para a modelagem conjunta de tempos de vida e medições longitudinais de escalas de sintomas, cujo suporte é o intervalo $[0,100]$. Para isso, após a transformação das respostas no intervalo unitário aberto, considerou-se para a explicação das variáveis limitadas o modelo de regressão beta inflacionado em zero (Ospina e Ferrari, 2012) com efeitos aleatórios. Em Rue et al. (2017) e Liu e Li (2016) a análise simultânea de respostas longitudinais multivariadas envolvendo uma medida limitada e uma medida que assume valores reais, e tempos de sobrevivência, é feita sob a perspectiva bayesiana.

Além de proporcionar uma descrição apropriada das respostas longitudinais, encontrar fatores de risco estatisticamente significativos e estimar a associação entre os processos investigados, a abordagem conjunta permite que as predições das probabilidades de sobrevivência sejam feitas de forma natural. 
Uma vez que os modelos são ajustados a um determinado conjunto de dados, as estimativas para as sobrevidas individuais podem ser atualizadas de forma dinâmica, utilizando toda a história do processo longitudinal até aquele instante junto com novas observações destas respostas (Rizopoulos, 2012).

Em muitos estudos a qualidade de vida é usada como um indicador de saúde para estabelecer a relação entre o custo e o benefício de um tratamento, com a finalidade de determinar seu valor social frente a outras prioridades da assistência médica. Já em pesquisas sobre o tratamento do câncer essa medida é usada como um importante fator prognóstico, auxiliando na tomada de decisões terapêuticas assertivas (Kypriotakis et al., 2016). Essas aplicações motivaram as abordagens metodológicas conduzidas nesta tese. Baseado em um estudo envolvendo pacientes oncológicos que deram entrada em unidades de terapia intensiva este trabalho tem como escopo principal: (i) estimar o impacto das evolução das trajetórias longitudinais de qualidade de vida na taxa de mortalidade, (ii) aprimorar a predição da sobrevida por meio da modelagem adequada dos dados e (iii) avaliar o potencial preditivo dos escores de qualidade de vida na estimação das sobrevidas individuais.

Considera-se a especificação de modelos conjuntos sob o paradigma frequentista, em situações nas quais as medidas longitudinais de qualidade de vida encontram-se continuamente distribuídas no intervalo unitário. Neste contexto, não encontra-se reportado na literatura os efeitos da má especificação da componente longitudinal na estimação da associação entre os processos investigados e nas predições da sobrevida. Embora a análise bayesiana tenha sido amplamente explorada na modelagem de dados longitudinais limitados e tempos de sobrevivência, essa abordagem é computacionalmente intensiva para um estudo criterioso das predições, não havendo evidências de investigações neste sentido em estudos sobre qualidade de vida.

O uso de procedimentos inferenciais frequentistas nesses tipos de aplicações pode gerar diversas dificuldades relacionadas aos algoritmos utilizados na estimação dos parâmetros e na avaliação dos seus erros padrão. Um objetivo secundário deste trabalho é propor metodologias computacionais que possibilitem a correção de problemas comuns de convergência, como falhas ou lentidão do processo iterativo. Esse tipo de investigação permite reduzir o alto tempo de processamento exigido nessa etapa, além de contribuir para a replicabilidade de análises computacionalmente intensivas. 


\subsection{Organização da tese}

Uma descrição detalhada do estudo que motivou este trabalho e uma análise exploratória das respostas longitudinais e de sobrevivência investigadas, são apresentadas no Capítulo 2. Uma introdução aos conceitos preliminares da modelagem conjunta é feita no Capítulo 3. O Capítulo 4 é dedicado à especificação de dois modelos conjuntos, com base nas distribuições beta (Ferrari e Cribari-Neto, 2004) e beta inflacionada em um (Ospina e Ferrari, 2012) para a descrição das respostas longitudinais, e no modelo de taxa de falhas proporcionais Weibull para a modelagem dos tempos de sobrevivência. O Capítulo 5 trata de uma análise computacional dos principais algoritmos empregados na estimação frequentista de modelos conjuntos. Uma proposta para a correção de problemas de convergência na estimação de máxima verossimilhança é discutida no Capítulo 6. No Capítulo 7 são apresentados os resultados de um estudo de simulação que avalia os efeitos da má especificação dos modelo longitudinal nas estimativas dos parâmetros. As metodologias desenvolvidas são aplicadas no Capítulo 8 para a análise do conjunto de dados descritos no Capítulo 2. Por fim, as discussões à respeito das contribuições deste trabalho e de propostas para a sua extensão são encontradas no Capítulo 9. 


\section{Capítulo 2}

\section{Motivação}

O conjunto de dados que motivou este trabalho é proveniente de um estudo de coorte prospectivo, realizado no Instituto do Câncer Dr. Octávio Frias de Oliveira - ICESP e na Fundação Pio XII - Hospital do Câncer de Barretos, ambos situados no Estado de São Paulo - Brasil. O objetivo da pesquisa foi descrever a sobrevida, a qualidade de vida relacionada à saúde e os anos de vida ajustados para a qualidade de vida (Qualitity - Adjusted Life Years, QALYs) de pacientes oncológicos admitidos em unidades de terapia intensiva. Este capítulo explora o conjunto de observações coletadas, destacando os principais aspectos que motivaram as metodologias desenvolvidas nesta tese.

\subsection{Dados ICESP}

O estudo foi conduzido nas UTIs oncológicas do ICESP e do Hospital do Câncer de Barretos. Os critérios para a inclusão dos participantes estavam condicionados à idade ( $\geq 18$ anos) e ao diagnóstico do câncer (provável ou comprovado). Efetuou-se a exclusão de indivíduos: com diagnóstico de câncer descartado após a admissão na UTI; com tempo de internação maior do que 24 horas; ou que não consentiram a participação no estudo. Esse conjunto de procedimentos, realizado nos períodos de março de 2010 à agosto de 2011, gerou uma amostra de 803 pacientes. Uma grande quantidade de dados foi coletada na admissão nas unidades de terapia intensiva, contemplando: os perfis demográficos e sociais dos participantes; informações relativas ao quadro clínico; ao histórico do câncer; à presença de comorbidades, entre outras variáveis. 
O delineamento da pesquisa incluiu o acompanhamento longitudinal dos pacientes, a fim de se considerar as seguintes medidas de desfecho:

i) A qualidade de vida relacionada à saúde (QVRS), aferida por meio de entrevistas realizadas nos períodos de 15 dias e 3, 6, 12, 18 meses após as altas das internações;

ii) Os tempos de sobrevivência de cada indivíduo, definidos como a diferença (em dias) entre a data de admissão na UTI e a ocorrência do óbito $(T)$ ou da censura $(C)$. Estes registros foram obtidos através da verificação dos status vitais de cada indivíduo, nos períodos preestabelecidos para a coleta e no tempo máximo de acompanhamento, fixado em 24 meses.

Uma descrição mais detalhada a respeito dos protocolos deste estudo pode ser encontrada em Silva (2015) e em Normilio-Silva et al. (2016).

\subsubsection{Escores de qualidade de vida}

A avaliação da qualidade de vida foi realizada por meio do instrumento EuroQol - 5 Dimensões (EQ-5D) (Group, 1990). Trata-se de um sistema descritivo que permite mensurar os benefícios gerados por diferentes tipos de intervenções terapêuticas. Esta ferramenta é composta por um questionário que abrange cinco dimensões associadas ao estado de saúde e bem estar geral: (I) Mobilidade, (II) Autocuidado, (III) Atividades habituais, (IV) Dor/Desconforto e (V) Ansiedade/Depressão. Cada categoria pode ser classificada segundo três níveis de percepção: 1- Sem problemas, 2- Alguns problemas e 3-Problemas severos.

As respostas qualitativas do questionário caracterizam o estado de saúde de um indivíduo. Desta forma, o EQ-5D é capaz de discriminar 243 perfis de saúde possíveis. No conjunto de dados analisado, os escores gerados nas entrevistas encontram-se convertidos em escalas numéricas, denominadas índices de utilidade (IU) ou utilidades. Estas medidas foram obtidas através de equações baseadas em técnicas econométricas e estimam as preferências de uma população por estados de saúde (Rabin et al., 2011; Lundberg et al., 1999). No estudo descrito, as escalas estão limitadas ao intervalo $[-0.54,1]$ e são interpretadas como segue: utilidades iguais a zero representam a morte; valores negativos representam estados de saúde considerados piores do que a morte; e utilidades iguais a um correspondem a um estado 
de saúde perfeito. Para fins de modelagem, os escores negativos foram mapeados a valores próximos de zero.

\subsection{Análise exploratória das respostas longitudinais}

No contexto dos cuidados intensivos, é razoável conjecturar que os sucessivos estados de saúde de um indivíduo evoluam de forma contínua no tempo. Esta suposição descreve bem o comportamento do processo biológico observado no estudo do ICESP, uma vez que não se esperam saltos na qualidade de vida dos pacientes em intervalos pequenos de tempo. Deste modo, embora cada medição longitudinal das utilidades provenha de uma coleção de 243 valores possíveis, tais escalas são tratadas como variáveis contínuas no intervalo $(0,1]$.

Em muitos problemas práticos a análise de respostas contínuas em intervalos unitários se baseia em transformações de dados. A ideia é mapear as observações originais no suporte das distribuições usadas na etapa de modelagem. Esta é uma abordagem de fácil implementação que deve ser aplicada com bastante critério, como argumenta-se a seguir:

i) Variáveis contínuas no intervalo $(0,1)$ são comumente mapeadas a valores reais, para então serem descritas através de modelos gaussianos (Kieschnick e McCullough, 2003). No entanto, esta estratégia nem sempre corrige a assimetria e heteroscedasticidade dos dados. Além disso, o uso de transformações altera a natureza das respostas, o que compromete a interpretação dos parâmetros.

ii) Quando os dados se distribuem de forma contínua nos intervalos $[0,1],(0,1]$ ou $[0,1)$, os pontos de fronteira são mapeados para o interior destes conjuntos. Logo, a modelagem das respostas pode ser baseada em distribuições como a beta, Kumaraswamy (Kumaraswamy, 1980), simplex (BarndorffNielsen e Jørgensen, 1991), beta retangular (Hahn, 2008), entre outras. Contudo, esta alternativa é indicada apenas em cenários com baixas ocorrências de zeros e uns. Proporções mais altas destas observações na amostra podem gerar dificuldades no processo de estimação dos parâmetros (Galvis et al., 2014; Hunger et al., 2012). 
Com o objetivo de verificar a viabilidade destas abordagens na modelagem dos dados do ICESP, consideram-se duas transformações do conjunto de medições dos índices de utilidade. Para descrevêlas denota-se por $y_{i j} \in(0,1)$ a medição longitudinal do $i$-ésimo indivíduo da amostra, em um tempo $j$. As $n_{i}$ observações do indivíduo $i$ coletadas em diferentes instantes $t_{j}$ são representadas pelo vetor $\boldsymbol{y}_{i}=\left(y_{i 1}, \ldots, y_{i n_{i}}\right)^{\top}$. Designa-se por $y_{i j}^{\prime}$ as observações de valores extremos do intervalo unitário, contidas em $\boldsymbol{y}_{i}$.

\section{Transformação de Smithson e Verkuilen (2006)}

A transformação proposta em Smithson e Verkuilen (2006) mapeia pontos de fronteira do intervalo $[0,1]$ para o interior deste conjunto. Nesse caso, os $m_{i}\left(m_{i} \leq n_{i}\right)$ elementos $y_{i j}^{\prime}$ do vetor $\boldsymbol{y}_{i}$ são transformados no intervalo $(0,1)$ da seguinte forma:

$$
y_{i j}^{\star}=\frac{y_{i j}^{\prime}(n-1)+0,5}{n}, \quad i=1, \ldots, n, \quad j=1, \ldots, m_{i},
$$

em que $\boldsymbol{y}_{i}^{\star}$ é o vetor obtido aplicando-se a transformação (2.1) nas observações $y_{i j}^{\prime}$ do vetor $\boldsymbol{y}_{i}$. No conjunto de dados do ICESP, em que todas as observações de fronteira são iguais a um, as transformações são dadas por

$$
y_{i j}^{\star}=1-0,5 / n, \quad i=1, \ldots, n, \text { e } j=1, \ldots, m_{i} .
$$

\section{Transformação Logito}

Algumas funções monótonas usadas para mapear dados observados no intervalo $(0,1)$ a valores reais são descritas em Atkinson (1985). Neste trabalho consideramos, para efeitos ilustrativos, a transformação logito dos índices de utilidade:

$$
y_{i j}^{\mathcal{L}}=\log \left(\frac{\tilde{y}_{i j}}{1-\tilde{y}_{i j}}\right), \quad i=1, \ldots, n, \text { e } j=1, \ldots, m_{i}
$$

em que

$$
\tilde{y}_{i j}= \begin{cases}y_{i j}, & \text { se } y_{i j} \in(0,1), \\ y_{i j}^{\star}, \quad \text { se } y_{i j} \in\{0,1\}, \quad i=1, \ldots, n, \text { e } j=1, \ldots, n_{i} .\end{cases}
$$

Desta forma, o vetor de observações transformadas $\boldsymbol{y}_{i}^{\mathcal{L}}=\left(y_{i 1}^{\mathcal{L}}, \ldots, y_{i n_{i}}^{\mathcal{L}}\right)^{\top}$ é constituído por elementos $y_{i j}^{\mathcal{L}} \in \mathbb{R}$. 


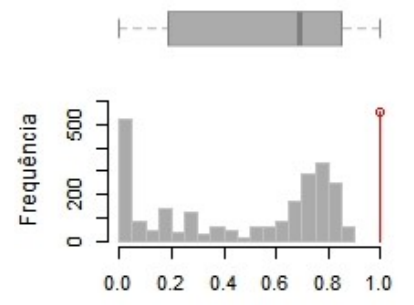

IU
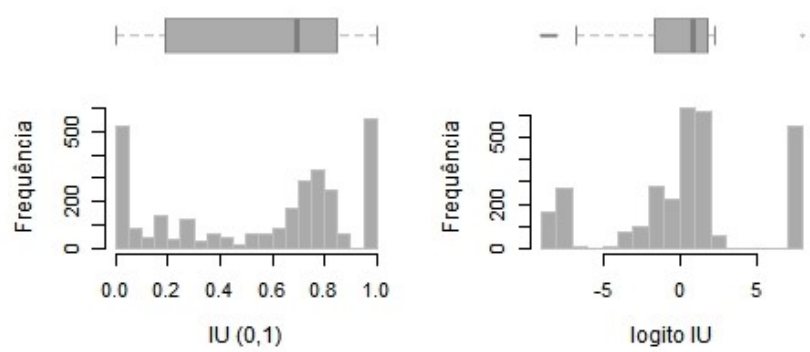

Figura 2.1: Boxplots e histogramas de frequência para as observações dos índices de utilidade, considerando: à esquerda, os dados em sua escala original (IU); ao centro, a transformação de Smithson e Verkuilen (2006) das medidas (IU (0,1)); e à direita, a transformação logito dos valores observados (logito IU).

Na Figura 2.1 encontram-se os histogramas de frequência e os boxplots dos índices de utilidade para os dados de ICESP observados em sua escala original, e considerando as transformações de Smithson e Verkuilen (2006) e logito, respectivamente. Na primeira ilustração, a barra vermelha indica o número de escores máximos na amostra. Estas medições correspondem a 19\% das observações. Verifica-se que os índices de utilidade se distribuem de forma assimétrica no intervalo unitário. Embora a transformação logito tenha atenuado a assimetria dos dados, a concentração de valores nos extremos do intervalo sugere que as respostas transformadas violam a suposição de normalidade.

Com relação à transformação de Smithson e Verkuilen (2006), as utilidades máximas são mapeadas a valores muito próximos de um. De acordo com Hunger et al. (2012), dependendo da quantidade de observações transformadas nessa região, é possível haver problemas de estabilidade na estimação de

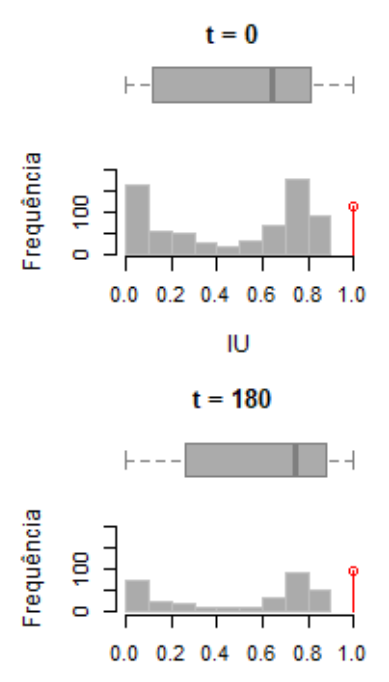

IU

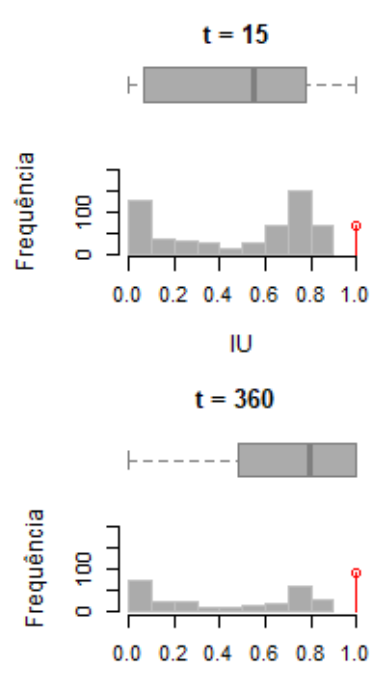

IU

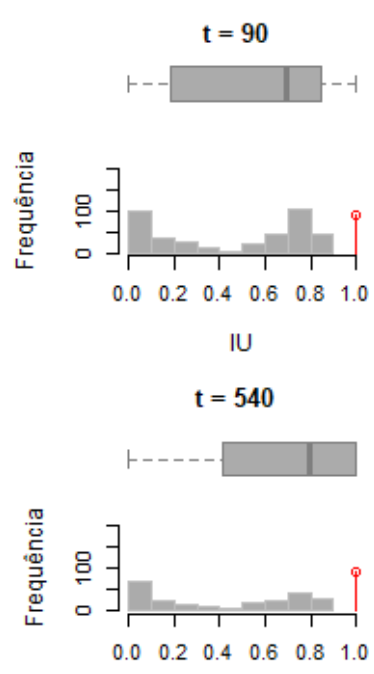

IU

Figura 2.2: Boxplots e histogramas de frequência para as observações dos IUs para os diferentes períodos do acompanhamento longitudinal. 
alguns modelos, como os modelos de regressão beta (Ferrari e Cribari-Neto, 2004), o que pode afetar a precisão das estimativas.

Na Figura 2.2 encontram-se os histogramas de frequência e os boxplots das medidas longitudinais para cada instante do acompanhamento. Observa-se que os dados mantêm uma distribuição assimétrica ao longo do tempo e que em todos os períodos do seguimento longitudinal uma proporção não desprezível de utilidades máximas foi registrada entre os pacientes.

As características exploradas indicam que a modelagem direta das respostas longitudinais é mais adequada nesta aplicação. O uso de distribuições contínuas, de suporte limitado e que acomodem observações de fronteira permite que os parâmetros do modelo sejam facilmente interpretados, algo desejável do ponto de vista médico. Além disso, segundo Galvis et al. (2014), essa abordagem pode ser particularmente vantajosa na análise de dados correlacionados, na qual uma má especificação pode exercer maior influência em resultados inferenciais.

Na Tabela 2.1 estão dispostos o número de indíviduos na amostra, as médias e os desvios padrão das utilidades observadas e a frequência relativa de índices de utilidade máximos (\%), para cada período do seguimento. Verifica-se que a qualidade de vida dos pacientes apresentou uma melhora ao longo do tempo, seguida por uma pequena redução na variabilidade das medidas. Um aumento na proporção de utilidades máximas na amostra pode ser observado, sinalizando que os estados de saúde dos pacientes avaliados melhoram após o tratamento intensivo.

Essas tendências devem ser analisadas com cuidado antes da modelagem dos dados, pois não levam em conta as omissões no conjunto de observações longitudinais. Durante o período do estudo foram registradas 18 omissões intermitentes e 503 perdas de acompanhamento. Isso resultou em uma redução

Tabela 2.1: Número de indíviduos na amostra, médias e desvios padrão dos IU's observados, e frequência relativa de IU's máximos (\%) para cada instante de coleta.

\begin{tabular}{c|ccc}
\hline Tempos de observação (dias) & $n$ & Média (Desvio Padrão) & IU máximo (\%) \\
\hline 0 & 803 & $0,51(0,36)$ & 14 \\
15 & 649 & $0,46(0,35)$ & 10 \\
90 & 481 & $0,55(0,36)$ & 19 \\
180 & 403 & $0,62(0,35)$ & 24 \\
365 & 337 & $0,68(0,32)$ & 27 \\
540 & 300 & $0,68(0,32)$ & 30 \\
\hline
\end{tabular}



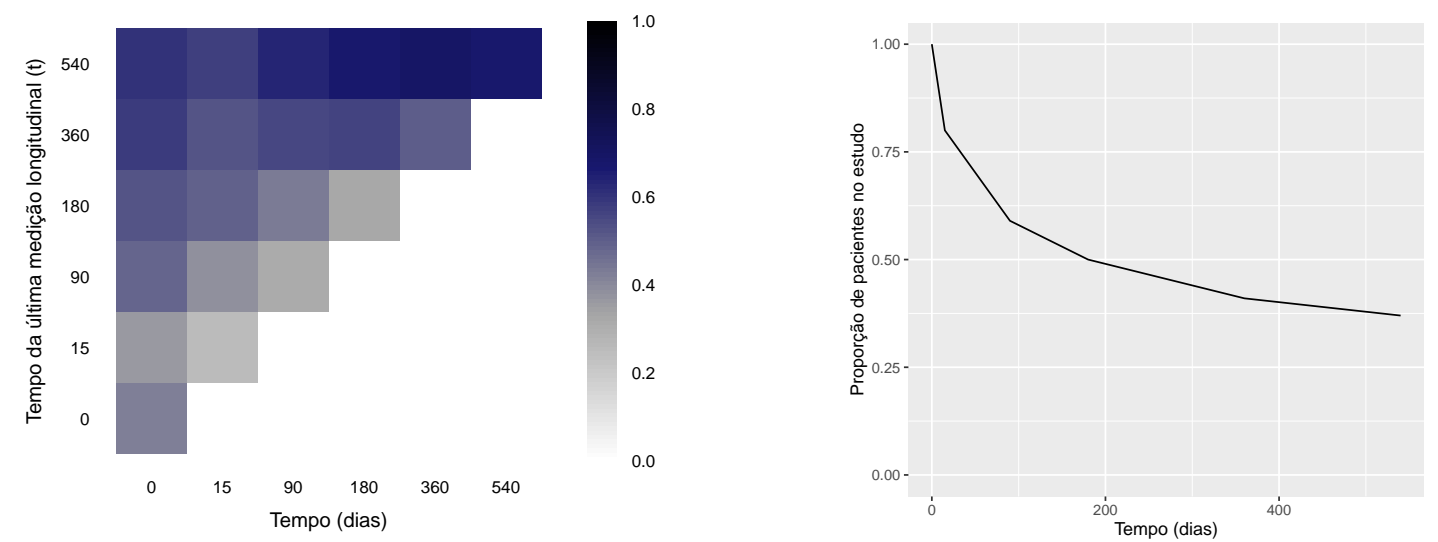

Figura 2.3: À esquerda, o mapa de calor para os IUs médios de pacientes estratificados segundo os tempos da última medição longitudinal ( $t$ ), em cada período de coleta dos dados. À direita, a evolução da proporção de pacientes no estudo no período do seguimento longitudinal.

de mais de $60 \%$ do tamanho amostral. Um aspecto importante do processo observado é que cerca de 98\% dos abandonos se devem ao óbito dos pacientes.

Para entender como as respostas longitudinais influenciam as omissões, estratificou-se os pacientes da amostra segundo os tempos da última medição longitudinal $(t)$. Para cada grupo obtido calculouse a média das utilidades observadas em períodos nos quais a coleta foi realizada. Essas informações encontram-se disponibilizadas no mapa de calor da Figura 2.3 (painel à esquerda). É possível constatar que: (i) quanto menor a utilidade média observada, menor a permanência dos indivíduos no estudo; (ii) em pacientes com dados incompletos $(t<540)$ houve uma piora gradual dos estados de saúde, uma vez que as utilidades médias nestes grupos são decrescentes; (iii) os casos completos ( $t=540)$ apresentaram os maiores índices de utilidade médios durante todo o acompanhamento longitudinal e uma trajetória crescente dos estados de saúde.

Esse comportamento sugere que existem pelo menos duas características que contribuem com o aumento dos abandonos: (i) um baixo valor de escore e (ii) uma evolução decrescente das medidas de utilidade dos pacientes. Para a análise longitudinal das respostas de qualidade de vida obtidas neste estudo, é fortemente recomendável considerar estratégias de modelagem que sejam apropriadas ao contexto dos dados incompletos. A exclusão desses casos fornece uma amostra de sobreviventes que pode não refletir com fidelidade aspectos importantes do processo investigado. Por outro lado, desprezar as omissões através do uso de métodos tradicionais poderá afetar a precisão das estimativas devido a redução do tamanho amostral (Molenberghs et al., 2014). 


\subsection{Escores de qualidade de vida como preditores da mortalidade}

Os tempos de sobrevivência observados são resultados de 24 meses de seguimento longitudinal dos pacientes. Nesse período, registraram-se 519 (65\%) óbitos e 284 (35\%) observações censuradas à direita, divididas em 267 (33\%) censuras do Tipo I e 17 (2\%) censuras aleatórias. A Figura 2.4 mostra a curva das estimativas de Kaplan-Meier (Kaplan e Meier, 1958) para a probabilidade de sobrevivência dos 803 pacientes em estudo. Uma alta mortalidade é notada nos instantes iniciais do acompanhamento, sendo atenuada ao longo do tempo. Além disso, nos períodos em que a curva de sobrevivência teve quedas mais acentuadas, menores índices de utilidade foram referidos pelos pacientes (o que é evidenciado na Figura 2.3). Esse comportamento indica que a evolução longitudinal dessas medidas tem o potencial de aprimorar a descrição do mecanismo de sobrevivência, podendo ser empregada na modelagem dos tempos até a ocorrência do óbito.

Para a aplicação dos escores de qualidade de vida como covariáveis dependentes do tempo é preciso considerar a sua natureza endógena, uma vez que a observação das utilidades de um paciente até um tempo $t$ implica na sua sobrevivência até este instante. No problema explorado, tanto os abandonos informativos no processo longitudinal quanto a endogeneidade no processo de sobrevivência podem ser devidamente acomodados por meio da modelagem conjunta das respostas, inserindo-se as trajetórias longitudinais da qualidade de vida no preditor linear do modelo de sobrevivência.

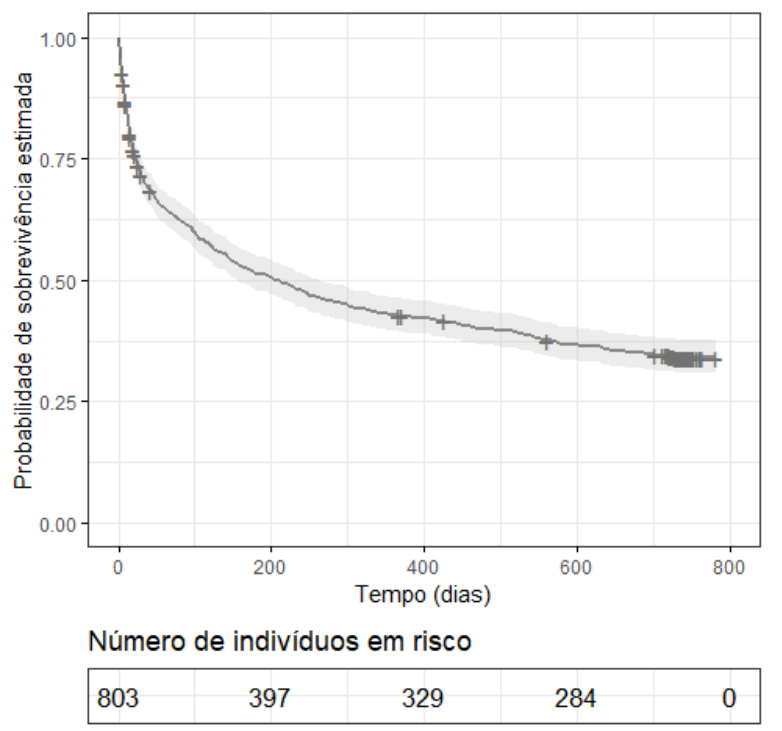

Figura 2.4: Estimativa de Kaplan-Meier para a probabilidade de sobrevivência dos 803 pacientes do estudo. 


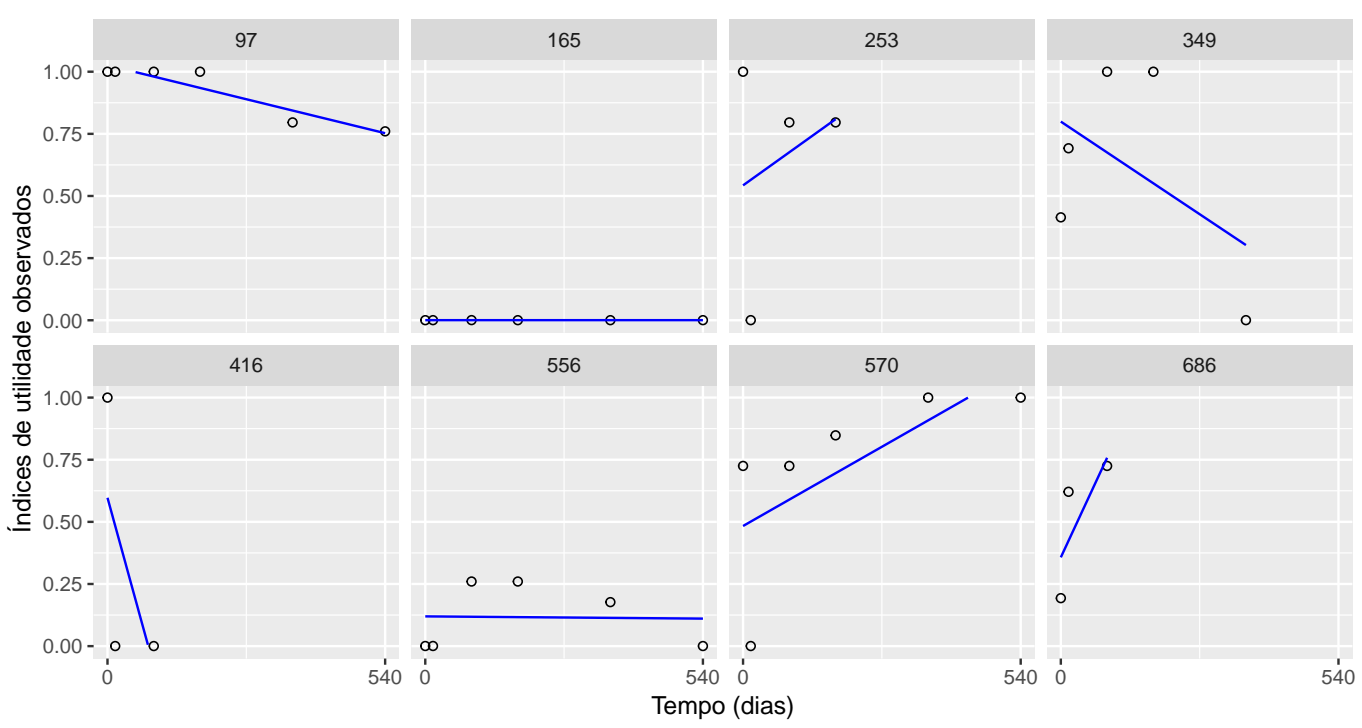

Figura 2.5: Observações longitudinais dos índices de utilidade (pontos abertos) e trajetórias individuais médias (retas azuis), obtidas através do método LOESS, para 8 indivíduos selecionados aleatoriamente na amostra do ICESP.

Em geral, é esperado que sobrevidas menores sejam atribuídas à indivíduos com valores baixos de utilidades ou com trajetórias longitudinais decrescentes. No entanto, o avanço nas áreas da terapia intensiva e oncologia vem proporcionando melhoras na evolução clínica dos pacientes, gerando padrões de observações problemáticos para a predição da sobrevivência. No conjunto de dados do ICESP, esses casos correspondem: (i) ao prolongamento da sobrevida de indivíduos que apresentam condições de saúde comprometidas durante todo o acompanhamento; e (ii) à pacientes que reportam saúde perfeita em uma ou mais ocasiões da coleta e morrem muito antes da conclusão do seguimento longitudinal.

Na Figura 2.5 encontram-se medições longitudinais das utilidades de oito indivíduos selecionados de forma aleatória na amostra. Embora os pacientes 165 e 556 tenham sido acompanhados até o final do estudo, a ocorrência de escores baixos ou próximos de zero pode resultar em predições pessimistas de sobrevivência. Por outro lado, os pacientes 253, 349 e 416 forneceram índices de utilidade máximos em algum dos períodos de coleta, mas vieram a óbito antes que o seguimento pudesse ser concluído.

Neste estudo as observações de escore máximo devem ser tratadas a parte. Esse tipo de medição pode evidenciar uma evolução natural da qualidade de vida ou ser uma observação pontual decorrente de externalidades não explicadas pelas variáveis em estudo. No primeiro caso, trajetórias crescentes ou decrescentes da resposta longitudinal são definidas de forma suave, descrevendo um processo que parece evoluir de forma contínua no tempo (por exemplo, para os pacientes 97 e 570); no segundo, os escores máximos são observados antes ou depois de medições relativamente menores (como para os pacientes 
253, 349 e 416).

Embora os índices de utilidade sejam respostas relatadas pelos pacientes (patient reported outcomes, PROs), e portanto passíveis de erros de aferição, considera-se que a sua incorporação no modelo de sobrevivência pode promover uma melhor descrição dos tempos de vida. Ao longo dos próximos capítulos, a formulação de modelos conjuntos é feita com o objetivo de acomodar as características descritas nas seções anteriores. A seguir, alguns conceitos preliminares relativos à modelagem conjunta de dados longitudinais e de sobrevivência são apresentados para o melhor entendimento das metodologias e análises desenvolvidas nesta tese. 


\section{Capítulo 3}

\section{Modelos conjuntos para dados longitudinais e tem- pos de sobrevivência}

Este capítulo trata de alguns elementos conceituais da modelagem conjunta de dados longitudinais e tempos de sobrevivência. A discussão é iniciada na Seção 3.1, apresentando-se as motivações para a realização de uma análise simultânea das respostas. As notações e terminologias da modelagem conjunta são introduzidas na Seção 3.2. Nas Seções 3.3, 3.4 e 3.5, consideram-se as especificações usuais para as componentes longitudinais, de sobrevivência e para a estrutura de ligação. Por fim, as abordagens propostas para a estimação são brevemente discutidas na Seção 3.6.

\subsection{Motivação para a modelagem conjunta}

Em pesquisas desenvolvidas em diferentes áreas do conhecimento, são cada vez mais comuns os estudos longitudinais que se ocupam com a coleta de (i) medidas repetidas de uma ou mais respostas, como marcadores biológicos relativos à progressão de uma doença ou condição médica; (ii) variáveis explicativas, que podem ou não ser dependentes do tempo e (iii) tempos até a ocorrência de um ou mais eventos (como o óbito, progressão da doença para um nível específico e abandono do estudo, entre outros).

Nos estudos sobre o Vírus da Imunodeficiência Humana (HIV), por exemplo, variáveis referentes ao tratamento, informações sociodemográficas e características fisiológicas dos pacientes são observadas em um estágio inicial da coleta de dados. Medições de biomarcadores relacionados aos estados imunológicos, como as contagens de células CD4 são realizadas ao longo de visitas clínicas subsequentes, de acordo com os protocolos estabelecidos pela pesquisa. O seguimento longitudinal viabiliza o monitoramento dos 
pacientes até o desenvolvimento da Síndrome da Imunodeficiência Adquirida (AIDS) ou até a ocorrência do óbito, permitindo o registro dos tempos até a observação destes eventos clínicos.

Em muitas aplicações, é usual que a modelagem de medidas longitudinais e tempos de sobrevivência seja feita separadamente, considerando as ferramentas estatísticas mais adequadas para a descrição de cada uma das respostas. Essa abordagem, contudo, pode ser bastante desafiadora em situações práticas, devido à natureza dos dados observados. Algumas limitações envolvendo este tipo de estratégia são enumeradas a seguir:

1. Sob a perspectiva do processo longitudinal, a ausência de medições após a ocorrência do evento caracteriza uma omissão devido ao abandono. Isso significa que, para uma sequência de medidas $y_{i 1}, \ldots, y_{i n_{i}}$ associadas a um indivíduo $i$, a omissão de uma observação $y_{i j}$, com $1 \leq j<n_{i}$, implica na omissão das observações subsequentes $y_{i j+1}, \ldots, y_{i n_{i}}$. Esta característica dificulta a análise das respostas, principalmente quando os motivos do abandono estão relacionados à evolução das medidas repetidas. Nestes casos, é possível que as observações omissas carreguem algum tipo de informação adicional a respeito do processo longitudinal. Segundo Molenberghs et al. (2014), desconsiderar esta particularidade dos dados pode comprometer o desempenho de procedimentos inferenciais, introduzindo viés nas estimativas dos parâmetros de regressão.

2. Do ponto de vista do processo de sobrevivência, uma atenção especial deve ser dada à descrição dos tempos de falha, condicionalmente às medidas longitudinais. Nestes casos, é recorrente o uso do modelo de Cox para variáveis dependentes do tempo (Kalbfleisch e Prentice, 2011). Esta formulação exige que algumas condições sejam satisfeitas pelas respostas longitudinais, o que restringe a aplicabilidade da metodologia em situações gerais. Mais especificamente:

a) Em um modelo de sobrevivência para variáveis dependentes do tempo, é necessário ter acesso aos valores das respostas longitudinais nos instantes de observação de cada evento. Entretanto, muitos estudos são planejados de modo que as coletas sejam feitas de forma intermitente, nos momentos preestabelecidos pelos protocolos de pesquisa. Uma solução para este problema é a imputação de dados para os valores longitudinais não observados. No método da última observação levada adiante, ou Last Value Carried Forward (LVCF), por exemplo, para 
cada indivíduo da amostra, a observação longitudinal faltante é substituída pela observação mais recente. Este tratamento dos dados considera tais variáveis como quantidades fixas em intervalos de tempo e sem erros de medições, o que pode ser inadequado em diversas situações, resultando em estimativas tendenciosas para os parâmetros do modelo (Prentice, 1982).

b) Outro aspecto a ser considerado está relacionado aos processos longitudinais gerados por variáveis internas (também chamadas de endógenas). De acordo com Kalbfleisch e Prentice (2011), estas respostas são resultados de um processo estocástico inerente ao indivíduo e estão diretamente associadas ao mecanismo de falha. Muitos biomarcadores usados em estudos clínicos, por exemplo, podem ser classificados dessa forma. Nessas circunstâncias o uso do modelo de Cox não é recomendado, já que sua especificação requer que as variáveis dependentes do tempo sejam externas (ou exógenas), ou seja, os valores observados em um instante $t>0$ não devem ser afetados pela ocorrência do evento em um instante $u<t$.

As dificuldades enfrentadas em análises independentes de medidas longitudinais e tempos de sobrevivência motivaram o desenvolvimento de metodologias para a modelagem conjunta, nas quais a associação entre as variáveis pode ser adequadamente estabelecida. Existem várias maneiras de se construir estes modelos: (i) através da formulação direta da distribuição conjunta das respostas; (ii) por meio de modelos condicionais baseados na fatoração da distribuição conjunta, como os modelos de seleção e os modelos de mistura de padrões (Fitzmaurice et al., 2008; Verbeke e Davidian, 2009; Molenberghs et al., 2014); ou (iii) através do uso de efeitos aleatórios para explicar a associação entre as variáveis. Este capítulo concentra-se na explanação da última classe de modelos, conhecidos como modelos de parâmetros compartilhados, modelos conjuntos para dados longitudinais e tempos de sobrevivência ou simplesmente modelos conjuntos.

A especificação de um modelo através da formulação de parâmetros compartilhados baseia-se na suposição de que um conjunto de variáveis não observáveis (efeitos aleatórios) influenciam os processos longitudinal e de sobrevivência, simultaneamente. Desta forma, a dependência entre as respostas é induzida por meio de variáveis latentes, que são compartilhadas através de uma estrutura de ligação. Essa classe tem sido bastante explorada na literatura, mediante o desenvolvimento de diversas extensões propostas. Um panorama geral a respeito das contribuições mais relevantes da área pode ser encontrado 
nas revisões bibliográficas conduzidas por Tsiatis e Davidian (2004), Gould et al. (2015) e Papageorgiou et al. (2019).

Devido à sua grande funcionalidade, os modelos conjuntos são empregados na resolução de problemas nas diversas áreas do conhecimento. Em particular, uma crescente adesão tem sido reportada na literatura médica nos últimos anos. Desenvolvimentos recentes em estudos sobre o HIV são discutidos em Viviani et al. (2014), Martins et al. (2016) e Wu et al. (2019); em Ibrahim et al. (2004), Yu et al. (2008), Król et al. (2016) e Cao et al. (2018) os modelos conjuntos são usados no contexto dos estudos oncológicos; aplicações envolvendo a doença de Alzheimer e outras demências foram exploradas em Li e Luo (2017), Rouanet et al. (2016) e Proust-Lima et al. (2019); e em Soto et al. (2020) uma análise conjunta é aplicada a dados sobre Insuficiência Cardíaca Congestiva (ICC).

Uma das razões para a popularização da metodologia é o aprimoramento dos resultados inferenciais quando comparado a formulações baseadas na análise independente das respostas. De acordo com Ibrahim et al. (2010), a incorporação da associação entre as variáveis promove uma descrição mais fiel dos dados, reduzindo o viés nas estimativas dos parâmetros. Assim, é possível evidenciar os efeitos de tratamentos e de fatores prognósticos relevantes para a pesquisa.

Outro aspecto positivo é que os modelos conjuntos constituem um importante instrumento de predição, que permite estimar probabilidades de sobrevivência de forma dinâmica, após cada medição da resposta longitudinal. No contexto das práticas clínicas essa ferramenta pode trazer uma série de contribuições, como a determinação de prognósticos mais precisos, a otimização de estratégias de tratamentos, a tomada de decisões médicas individualizadas e o desenvolvimento de triagens mais eficazes (Rizopoulos, 2011; Rizopoulos et al., 2017; Papageorgiou et al., 2019).

\subsection{Notações e conceitos básicos}

Considere uma amostra aleatória constituída por $n$ indivíduos para os quais deseja-se observar o tempo até a ocorrência de um evento de interesse, denotado por $T_{i}^{*}(i=1, \ldots, n)$. Seja $T_{i}$ a variável aleatória representando o tempo efetivamente observado, definido como o mínimo entre o tempo de censura $C_{i}$ e $T_{i}^{*}$ e $\delta_{i}=I\left(T_{i}^{*} \leq C_{i}\right)$ a variável indicadora do evento. Denota-se por $y_{i}(t)$ o valor da covariável endógena dependente do tempo, em um instante $t$. Esta variável é observada em diferentes 
ocasiões $t_{i j}$ nas quais as coletas são realizadas, resultando em um conjunto de medidas longitudinais $\boldsymbol{y}_{i}=\left\{y_{i}\left(t_{j}\right) ; j=1, \ldots, n_{i}\right\}$. Representa-se por $m_{i}(t)$ o verdadeiro processo longitudinal associado ao indivíduo $i$ em um tempo $t$ e por $m_{i j}$ o valor correspondente no instante $t_{j}$. Para cada indivíduo são observados os vetores de variáveis explicativas $\boldsymbol{x}_{i}$ e $\boldsymbol{w}_{i}$, que podem ser dependentes do tempo, desde que sejam externas no sentido de Kalbfleisch e Prentice (2011).

Como mencionado anteriormente, um modelo conjunto é constituído (i) por um submodelo longitudinal, (ii) um submodelo de sobrevivência e (iii) uma estrutura de ligação entre as respostas. A seguir, resume-se algumas dessas especificações. Embora uma grande variedade de modelos conjuntos tenham sido explorados na literatura, neste capítulo consideram-se apenas as formulações usuais para as respostas, condicional aos efeitos aleatórios: um modelo linear gaussiano com efeitos mistos para as medidas longitudinais e um modelo de taxas de falhas proporcionais para os tempos de sobrevivência. Em ambos os casos, assume-se que as variáveis resposta são unidimensionais.

\subsection{Modelagem da componente longitudinal}

A resposta longitudinal para o $i$-ésimo indivíduo no instante $t$ pode ser modelada como

$$
y_{i}(t)=m_{i}(t)+e_{i}(t)
$$

em que $m_{i}(t)=\mu_{i}(t)+w_{i}(t)$ denota os verdadeiros valores da trajetória longitudinal nos instantes $t \geq 0, \quad w_{i}(t)$ acomoda termos que contêm traços latentes associados ao $i$ - ésimo indivíduo e $e_{i}(t)$ denota o erro de medida no instante $t$. Em geral, assume-se que $e_{i}(t)$ são normalmente distribuídos, com média zero. Uma forma simples de se modelar a resposta média é através da forma linear

$$
\mu_{i}(t)=\boldsymbol{x}_{i}(t)^{\top} \boldsymbol{\beta}
$$

em que $\boldsymbol{\beta}=\left(\beta_{1}, \ldots, \beta_{p 1}\right)^{\top}$ denota o vetor de efeitos fixos, relativo às $p_{1}$ variáveis explicativas contidas no vetor $\boldsymbol{x}_{i}(t)$. Especificações mais elaboradas podem ser utilizadas considerando-se estruturas não lineares ou não paramétricas de dependência do tempo, ou através da inclusão de termos de interação. Com relação ao processo latente, em geral assume-se que

$$
w_{i}(t)=f(t)^{\top} \boldsymbol{b}_{i},
$$


em que $f(t)$ é um vetor de funções suaves do tempo e $\boldsymbol{b}_{i}$ é um vetor de efeitos aleatórios associados ao indivíduo $i$. Usualmente, postula-se que o vetor $\boldsymbol{b}_{i}$ é normalmente distribuído, com média $\mathbf{0}$ e matriz de covariâncias $\Sigma$.

\subsection{Modelagem da componente de sobrevivência}

Em geral, a taxa para a ocorrência do evento é modelada por meio do modelo semiparamétrico de Cox (Cox, 1972), com função taxa de falha para o $i$-ésimo indivíduo dada por

$$
h_{i}\left(t \mid \boldsymbol{\omega}_{i}(t)\right)=h_{0}(t) \exp \left(\gamma^{\top} \boldsymbol{\omega}_{i}(t)+\alpha m_{i}(t)\right)
$$

em que $h_{0}(t)$ denota a componente não paramétrica do modelo, $\boldsymbol{\omega}_{i}(t)=\left(\omega_{i 1}(t), \ldots, \omega_{i p 2}(t)\right)^{\top}$ representa um vetor de parâmetros associados às $p_{2}$ variáveis de base contidas no vetor $\boldsymbol{\omega}_{i}(t)$ e $\alpha$ é o parâmetro que mede o efeito do verdadeiro valor da resposta longitudinal no instante t na taxa de ocorrência do evento.

No contexto dos modelos conjuntos, a abordagem semiparamétrica pode levar à subestimação dos erros padrão das estimativas (Hsieh et al., 2006). Uma alternativa para evitar estes problemas é utilizar uma função de taxa de falha basal que corresponde a algum modelo paramétrico, como os modelo Weibull ou exponencial. Uma outra solução é considerar especificações paramétricas mais flexíveis para $h_{0}(t)$, atavés de funções constantes por partes ou de splines (Whittemore e Keller, 1986; Herndon e Harrell Jr, 1990; Rosenberg, 1995).

\subsection{Estruturas de ligação}

A forma usual de associação entre o processo longitudinal e de sobrevivência assume que o risco de um evento em um instante $t$ depende do verdadeiro valor da covariável neste instante. Embora este tipo de parametrização pareça atrativa em muitas situações, a forma funcional da ligação pode gerar resultados imprecisos ou interpretações não consistentes com o problema investigado. A seguir, destacam-se algumas parametrizações alternativas à empregada usualmente. Todas elas podem ser expressas através da seguinte forma geral

$$
h_{i}(t)=h_{0}(t) \exp \left(\boldsymbol{\gamma}^{\top} \boldsymbol{\omega}_{i}(t)+f\left\{m_{i}(t), \boldsymbol{b}_{i}, \boldsymbol{\omega}_{i 2}(t) ; \boldsymbol{\alpha}\right\}\right),
$$


em que $f(\cdot)$ é uma função do verdadeiro valor do marcador $m_{i}(t)$, dos efeitos aleatórios $\boldsymbol{b}_{i}$, de covariáveis extras representadas pelo vetor $\boldsymbol{\omega}_{i}^{*}(t)=\left(\omega_{i 1}^{*}(t), \ldots, \omega_{i p 2}^{*}(t)\right)^{\top}$ e de $\boldsymbol{\alpha}$, que denota o vetor de parâmetros de associação.

1. Declive: Esta parametrização permite acomodar situações nas quais dois pacientes apresentam o mesmo valor para a resposta longitudinal em um instante $t$, mas diferem na taxa de variação do processo observado. Neste caso, postula-se que o risco de um evento depende do valor da trajetória e da sua derivada no potno $t$, ou seja

$$
h_{i}(t)=h_{0}(t) \exp \left(\boldsymbol{\gamma}^{\top} \boldsymbol{\omega}_{i}+\alpha_{1} m_{i}(t)+\alpha_{2} \frac{d}{d t} m_{i}(t)\right) .
$$

A interpretação do parâmetro $\alpha_{1}$ é a mesma da parametrização convencional, ao passo que $\alpha_{2}$ mede a associação entre o processo de sobrevivência e a taxa de variação da resposta longitudinal.

2. Efeito cumulativo: O efeito cumulativo do processo longitudinal na avaliação do risco pode ser uma suposição mais realista para algumas aplicações. Uma abordagem para considerar este efeito da resposta longitudinal é dada por

$$
h_{i}(t)=h_{0}(t) \exp \left(\boldsymbol{\gamma}^{\top} \boldsymbol{\omega}_{i}+\alpha \int_{0}^{t} m_{i}(s) d s\right)
$$

em que a área sob a trajetória longitudinal até o instante $t$ é considerada como uma boa medida resumo da trajetória completa e $\alpha$ mede a sua associação com o processo de sobrevivência.

3. Valor defasado: Em situações nas quais o risco para o evento em um instante $t$ depende do valor da variável longitudinal em um instante $t-c$, uma parametrização proposta é dada por

$$
h_{i}(t)=h_{0}(t) \exp \left(\boldsymbol{\gamma}^{\top} \boldsymbol{\omega}_{i}+\boldsymbol{\alpha}\left\{\boldsymbol{\omega}_{i}^{*} \times m_{i}(t-c)_{+}\right\}\right)
$$

em que $(t-c)_{+}=\max (t-c, 0)$ e a covariável $\omega_{i}^{*}$ é usada para acomodar termos de interações em $m_{i}(t-c)_{+} \cdot$

4. Efeitos aleatórios: Uma abordagem comumente utilizada na modelagem conjunta considera so- 
mente o uso de efeitos aleatórios na componente longitudinal, ou seja,

$$
h_{i}(t)=h_{0} \exp \left(\boldsymbol{\gamma}^{\top} \boldsymbol{\omega}_{i}+\boldsymbol{\alpha}^{\top} \boldsymbol{b}_{i}\right)
$$

Nesta formulação, pacientes que possuem um nível baixo/elevado da medida longitudinal no início do estudo (intercepto) ou apresenta uma evolução lenta/acentuada da sua trajetória (coeficiente angular), são mais propícios à experimentarem o evento.

\subsection{Estimação}

Na literatura existem duas abordagens consolidadas para a análise conjunta de dados longitudinais e de sobrevivência. São elas: a modelagem em duas etapas e a modelagem baseada na função de verossimilhança conjunta e discutidas a seguir. A modelagem em duas etapas consiste em: (i) especificar um modelo para a descrição das respostas longitudinais, obtendo-se as estimativas dos valores omissos das trajetórias individuais e (ii) imputar as respostas estimadas na função de verossimilhança parcial de Cox, em cada instante de falha. Embora a abordagem inferencial descrita seja de fácil implementação, Dafni e Tsiatis (1998), Tsiatis e Davidian (2001) e Sweeting e Thompson (2011) mostram em uma série de estudos de simulação que essa prática introduz viés nos parâmetros estimados. Por essa razão, a principal estratégia adotada em modelagens conjuntas é a formulação da função de verossimilhança conjunta.

A abordagem que usa a função de verossimilhança conjunta fundamenta-se na distribuição conjunta das respostas longitudinais e dos tempos de sobrevivência. Sob o paradigma frequentista, os procedimentos inferenciais são baseados na maximização da verossimilhança. As propriedades assintóticas destes estimadores, sob uma especificação semi-paramétrica do modelo de riscos proporcionais, é estudada em Zeng et al. (2005). Em geral, devido à complexidade dos modelos envolvidos, as estimativas de máxima verossimilhança não podem ser obtidas de forma analítica. Uma característica desse tipo de abordagem é que as integrais relativas aos efeitos aleatórios e à função de sobrevivência geram um alto custo computacional na etapa de estimação. Este fato muitas vezes minimiza a aplicabilidade dos procedimentos inferenciais frequentistas, sobretudo em modelos de maior complexidade. Uma discussão detalhada a respeito dos aspectos computacionais da estimação de máxima verossimilhança, no contexto dos modelos conjuntos, é feita no Capítulo 5. 


\subsection{Predições das probabilidades de sobrevivência}

Após o ajuste de um modelo conjunto à uma amostra aleatória $\mathcal{D}_{n}=\left\{\left(T_{1}, \delta_{1}, \boldsymbol{y}_{1}\right), \ldots,\left(T_{n}, \delta_{n}, \boldsymbol{y}_{n}\right)\right\}$, as informações obtidas podem ser usadas para predizer as probabilidades de sobrevivência de um novo indivíduo $i$, associado a um conjunto de medições longitudinais $\mathcal{Y}_{i}(t)=\left\{y_{i}(s) ; 0 \leq s \leq t\right\}$. Nesse contexto, deseja-se calcular a probabilidade condicional de se sobreviver até um tempo $u>t$, dada a sobrevivência até o instante $t$, ou seja

$$
\pi_{i}(u \mid t)=P\left(T_{i}^{*} \geq u \mid T_{i}^{*}>t, \boldsymbol{y}_{i}, \boldsymbol{\omega}_{i}, \boldsymbol{x}_{i}, \mathcal{D}_{n} ; \boldsymbol{\theta}\right), \quad t \geq 0
$$

em que $\boldsymbol{\theta}=\left(\boldsymbol{\theta}_{t}^{\top}, \boldsymbol{\theta}_{y}^{\top}, \boldsymbol{\theta}_{b}^{\top}\right)^{\top}$ denota o vetor de parâmetros do modelo conjunto, composto pelos parâmetros $\boldsymbol{\theta}_{t}$ do submodelo de sobrevivência, $\boldsymbol{\theta}_{y}$ do submodelo longitudinal e $\boldsymbol{\theta}_{b}$ da matriz de covariâncias dos efeitos aleatórios.

Essa definição mostra que a probabilidade condicional de sobrevivência tem uma natureza dinâmica. Quando novas observações longitudinais são coletadas para um indivíduo $i$, em um instante $t^{\prime}>t$, é possível atualizar as predições, obtendo $\pi_{i}\left(u \mid t^{\prime}\right)$. Para simplificar a notação, o condicionamento nas covariáveis $\omega_{i}$ e na amostra $\mathcal{D}_{n}$ é omitido nas próximas expressões.

A probabilidade (3.10) pode ser reescrita como (Rizopoulos, 2012),

$$
\begin{aligned}
P\left(T_{i}^{*} \geq u \mid T_{i}^{*}>t, \mathcal{Y}_{i}(t) ; \boldsymbol{\theta}\right) & =\int P\left(T_{i}^{*} \geq u \mid T_{i}^{*}>t, \mathcal{Y}_{i}(t), \boldsymbol{b}_{i} ; \boldsymbol{\theta}\right) p\left(\boldsymbol{b}_{i} \mid T_{i}^{*}>t, \mathcal{Y}_{i}(t) ; \boldsymbol{\theta}\right) d \boldsymbol{b}_{i} \\
& =\int P\left(T_{i}^{*} \geq u \mid T_{i}^{*}>t, \boldsymbol{b}_{i} ; \boldsymbol{\theta}\right) p\left(\boldsymbol{b}_{i} \mid T_{i}^{*}>t, \mathcal{Y}_{i}(t) ; \boldsymbol{\theta}\right) d \boldsymbol{b}_{i} \\
& =\int \frac{S_{i}\left(u \mid \mathcal{M}_{i}\left(u, \boldsymbol{b}_{i}, \boldsymbol{\theta}\right) ; \boldsymbol{\theta}\right)}{S_{i}\left(t \mid \mathcal{M}_{i}\left(t, \boldsymbol{b}_{i}, \boldsymbol{\theta}\right) ; \boldsymbol{\theta}\right)} p\left(\boldsymbol{b}_{i} \mid T_{i}^{*}>t, Y_{i}(t) ; \boldsymbol{\theta}\right) d \boldsymbol{b}_{i} .
\end{aligned}
$$

Considerando a equação (3.11), Rizopoulos (2011) propôs um estimador para $\pi_{i}(u \mid t)$, dado por

$$
\hat{\pi}_{i}(u \mid t)=\frac{S_{i}\left(u \mid \mathcal{M}_{i}\left(u, \hat{\boldsymbol{b}}_{i}^{(t)}, \hat{\boldsymbol{\theta}}\right) ; \hat{\boldsymbol{\theta}}\right)}{S_{i}\left(t \mid \mathcal{M}_{i}\left(t, \hat{\boldsymbol{b}}_{i}^{(t)}, \hat{\boldsymbol{\theta}}\right) ; \hat{\boldsymbol{\theta}}\right)},
$$

em que $\hat{\boldsymbol{b}}_{i}^{(t)}$ denota a predição de $\boldsymbol{b}_{i}$ no instante $t$. A inferência para os efeitos aleatórios é feita sob o 
paradigma bayesiano, com base na sua distribuição a posteriori

$$
\begin{aligned}
p\left(\boldsymbol{b}_{i} \mid T_{i}^{*}>t, \mathcal{Y}_{i}(t) ; \hat{\boldsymbol{\theta}}\right) & =\frac{P\left(T_{i}^{*}>t, \mathcal{Y}_{i}(t), \boldsymbol{b}_{i} ; \widehat{\boldsymbol{\theta}}\right)}{P\left(T_{i}>t, \mathcal{Y}_{i}(t) ; \widehat{\boldsymbol{\theta}}\right)} \\
& =\frac{P\left(T_{i}^{*}>t \mid \boldsymbol{b}_{i} ; \widehat{\boldsymbol{\theta}}\right) p\left(\mathcal{Y}_{i}(t) \mid \boldsymbol{b}_{i} ; \hat{\boldsymbol{\theta}}\right) p\left(\boldsymbol{b}_{i} \mid \hat{\boldsymbol{\theta}}\right)}{\int P\left(T_{i}^{*}>t, \mathcal{Y}_{i}(t), \boldsymbol{b}_{i} ; \widehat{\boldsymbol{\theta}}\right) d \boldsymbol{b}_{i}} \\
& \propto P\left(T_{i}^{*}>t, \mathcal{Y}_{i}(t), \boldsymbol{b}_{i} ; \widehat{\boldsymbol{\theta}}\right)
\end{aligned}
$$

$\operatorname{com} P\left(T_{i}^{*}>t \mid \boldsymbol{b}_{i} ; \widehat{\boldsymbol{\theta}}\right)=S_{i}\left(t \mid \mathcal{M}_{i}\left(t, \hat{\boldsymbol{b}}_{i}, \hat{\boldsymbol{\theta}}\right) ; \hat{\boldsymbol{\theta}}\right) \mathrm{e}$

$$
p\left(\mathcal{Y}_{i}(t) \mid \boldsymbol{b}_{i} ; \hat{\boldsymbol{\theta}}\right)=\prod_{j=1}^{n_{i}(t)} p\left(y_{i j} \mid \boldsymbol{b}_{i} ; \hat{\boldsymbol{\theta}}\right),
$$

em que $n_{i}(t)$ é o número de observações longitudinais do indivíduo $i$ até o instante $t$. Neste trabalho, considera-se o estimador empírico de Bayes

$$
\hat{\boldsymbol{b}}_{i}^{(t)}=\underset{\boldsymbol{b}_{i}}{\operatorname{argmax}} \log p\left(\boldsymbol{b} \mid T_{i}, \delta_{i}, \boldsymbol{y}_{i} ; \hat{\boldsymbol{\theta}}\right) .
$$




\section{Capítulo 4}

\section{Modelos conjuntos para dados longitudinais con- tínuos no intervalo unitário e tempos de sobre- vivência}

Este capítulo trata da formulação de modelos conjuntos para situações nas quais, para cada unidade amostral, são observadas respostas longitudinais restritas ao intervalo $(0,1]$ e tempos de sobrevivência. As metodologias propostas foram motivadas pela descrição de características das medidas de qualidade de vida do conjunto de dados do ICESP, detalhado no Capítulo 2. Nas Seções 4.1 e 4.2 encontramse as especificações para as componentes de sobrevivência e longitudinal. A estimação de máxima verossimilhança dos modelos conjuntos é discutida na Seção 4.3. As contribuições da abordagem proposta na predição da qualidade de vida e das sobrevidas individuais no estudo do ICESP é abordada na Seção 4.4. E na Seção 4.5, discute-se uma metodologia para geração de erros padrão para as predições de sobrevivência, baseada na reamostragem do tipo bootstrap balanceada.

\subsection{Submodelo de sobrevivência}

Considere uma amostra composta por $n$ indivíduos para os quais deseja-se observar o tempo até a ocorrência de um evento de interesse, denotado por $T_{i}^{*}(i=1, \ldots, n)$. Seja $T_{i}$ a variável aleatória representando o tempo efetivamente observado, definido como o mínimo entre o tempo de censura $C_{i}$ e $T_{i}^{*}$, e $\delta_{i}=I\left(T_{i}^{*} \leq C_{i}\right)$ a variável indicadora do evento. Denota-se por $y_{i}(t)$ o valor da covariável endógena dependente do tempo, em um instante $t$. Esta variável, observada em diferentes ocasiões $t_{i j}$, resulta no conjunto de medições longitudinais $\boldsymbol{y}_{i}=\left\{y_{i}\left(t_{j}\right) ; j=1, \ldots, n_{i}\right\}$. Representa-se por $m_{i}(t)$ o verdadeiro processo longitudinal associado ao indivíduo $i$ no tempo $t$. 
Para medir o efeito do processo longitudinal no risco para o evento, postula-se um modelo caracterizado por uma taxa de falha dada por

$$
\begin{aligned}
h_{i}\left(t \mid \mathcal{M}_{i}(t), \boldsymbol{\omega}_{i}\right) & =\lim _{d t \rightarrow 0} P\left(t \leq T_{i}^{*} \leq t+d t \mid T_{i}^{*} \geq t, \mathcal{M}_{i}(t), \boldsymbol{\omega}_{i}\right) / d t \\
& =h_{0}(t) \cdot \exp \left\{\boldsymbol{\omega}_{i}^{\top} \boldsymbol{\gamma}+\alpha m_{i}(t)\right\}, \quad t>0, \quad i=1, \ldots, n
\end{aligned}
$$

em que $\mathcal{M}_{i}(t)=\left\{m_{i}(s) ; 0 \leq s<t\right\}$ representa a história do verdadeiro processo longitudinal (não observável) até o instante $t, h_{0}(t)$ denota a função de taxa de falha basal, $\boldsymbol{\omega}_{i}=\left(\omega_{i 1}, \ldots, \omega_{i q}\right)^{\top}$ é um vetor de covariáveis basais, $\gamma=\left(\gamma_{1}, \ldots, \gamma_{q}\right)^{\top}$ é o vetor de coeficientes de regressão correspondente e $\alpha$ é um parâmetro que mede a intensidade da associação entre o processo longitudinal e a taxa na qual os eventos ocorrem.

No modelo (4.1) o risco de ocorrência do evento para um indivíduo $i$ depende pontualmente da trajetória longitudinal descrita em $\mathcal{M}_{i}(t)$. Isso significa que a sua avaliação em um tempo $t$ exige o conhecimento do processo longitudinal somente naquele instante. Por outro lado, para a análise da função de sobrevivência

$$
\begin{aligned}
S_{i}\left(t \mid \mathcal{M}_{i}(t), \boldsymbol{\omega}_{i}\right) & =P\left(T_{i}^{*}>t \mid \mathcal{M}_{i}(t), \boldsymbol{\omega}_{i}\right) \\
& =\exp \left(-\int_{0}^{t} h_{0}(s) \cdot \exp \left\{\omega_{i}^{\top} \boldsymbol{\gamma}+\alpha m_{i}(s)\right\} d s\right)
\end{aligned}
$$

é preciso avaliar toda a história delineada em $\mathcal{M}_{i}(t)$ no intervalo $[0, t)$. As informações longitudinais referentes a cada unidade amostral consistem em um conjunto discreto de observações, que não são suficientes para este fim. Por esta razão, para descrever o processo de sobrevivência acomodando-se a endogeneidade de variáveis relevantes para o estudo, é importante considerar a modelagem adequada de suas trajetórias longitudinais.

Apresenta-se a seguir a especificação de dois modelos longitudinais para dados continuamente distribuidas em intervalos unitários. A primeira abordagem trata da modelagem direta de variáveis contidas no intervalo $(0,1)$. Já a segunda tem a finalidade de acomodar observações de pontos extremos do intervalo através de um modelo inflacionado. 


\subsection{Submodelos longitudinais}

\subsubsection{Modelo de regressão beta com efeitos aleatórios}

Nesta seção, as respostas longitudinais restritas ao intervalo $(0,1)$ são descritas através da distribuição beta, considerando a parametrização proposta em Ferrari e Cribari-Neto (2004). Esta família de distribuições é bastante conhecida pela flexibilidade na modelagem de dados com valores em um intervalo limitado, uma vez que sua densidade pode assumir diferentes formas, a depender dos parâmetros que a indexam. A função densidade de probabilidade de uma variável aleatória $Y$ com distribuição beta, em termos da parametrização considerada, é dada por

$$
f_{B}(y \mid \mu, \phi)=\frac{\Gamma(\mu+\phi)}{\Gamma(\mu \phi) \Gamma((1-\mu) \phi)} y^{\mu \phi-1}(1-y)^{(1-\mu) \phi-1}, \quad 0<y<1,
$$

em que $0<\mu<1$ representa a média da resposta $Y, \phi>0$ e $\Gamma(\cdot)$ é a função gama.

Denote por $y_{i j} \in(0,1)$ o valor da resposta longitudinal do $i$-ésimo indivíduo da amostra, em um instante $t_{j}$, e seja $\boldsymbol{y}_{i}=\left(y_{i 1}, \ldots, y_{i n_{i}}\right)^{\top}$ o vetor com as $n_{i}$ observações do indivíduo $i$. Condicional ao vetor de efeitos aleatórios $\boldsymbol{b}_{i}=\left(b_{0 i}, b_{1 i}\right)^{\top}$, assume-se que as respostas $y_{i j}$ são independentes e seguem distribuição beta com média $m_{i j}$ e parâmetro de precisão $\phi$. O valor médio $m_{i}(t)$ é modelado em termos de um preditor linear, através da ligação logito

$$
\log \left(\frac{m_{i}(t)}{1-m_{i}(t)}\right)=\boldsymbol{x}_{i}^{\top}(t) \boldsymbol{\beta}+\boldsymbol{z}_{i}^{\top}(t) \boldsymbol{b}_{i}
$$

em que $\boldsymbol{\beta}=\left(\beta_{1}, \ldots, \beta_{p}\right)$ é o vetor de efeitos fixos correspondente às variáveis explicativas $\boldsymbol{x}_{i}(t)=$ $\left(x_{i 1}(t), \ldots, x_{i p}(t)\right)^{\top}$ e $z_{i}(t)=\left(z_{i 1}(t), z_{i 2}(t)\right)^{\top}$ é o vetor de variáveis explicativas associadas a $\boldsymbol{b}_{\boldsymbol{i}}$. Supõese que os efeitos aleatórios relativos ao $i$-ésimo indivíduo têm distribuição normal multivariada com vetor de médias 0 e matriz de covariâncias $\boldsymbol{G}$.

As respostas limitadas, tais como os escores de qualidade de vida, geralmente apresentam menor variabilidade quando sua média se aproxima dos extremos do intervalo (Kieschnick e McCullough, 2003). A natureza heteroscedástica dessas observações pode ser explicada pelo modelo de regressão beta. Note que a variância condicional 


$$
\operatorname{Var}\left(Y_{i}(t) \mid \boldsymbol{b}_{i}\right)=\frac{m_{i}(t)\left(1-m_{i}(t)\right)}{1+\phi}
$$

assume uma forma quadrática que depende do valor médio das respostas, e consequentemente, das variáveis explicativas a elas associadas. Por essa razão, para as análises realizadas neste trabalho considera-se suficiente assumir que o parâmetro de precisão $\phi$ seja constante.

\subsubsection{Modelo de regressão beta inflacionado em um com efeitos aleatórios}

Embora muitas aplicações envolvam variáveis que são medidas continuamente no intervalo $(0,1)$, é comum que esses dados limitados também contenham observações de pontos extremos (zeros e uns). Dependendo da proporção na qual tais valores ocorram, a distribuição beta pode não ser uma opção adequada para descrevê-los (Hunger et al., 2011; Galvis et al., 2014), sendo necessário recorrer ao uso de modelos que capturem a massa de probabilidade na fronteira do intervalo unitário.

Uma maneira natural de se fazer isso é proposta em Ospina e Ferrari (2012). A mistura de duas distribuições é utilizada considerando: a distribuição beta (4.3) para modelar as respostas contínuas no intervalo $(0,1)$ e uma distribuição degenerada para descrever as observações dos pontos de fronteira deste conjunto. Assim, a função densidade de probabilidade de uma variável $Y$, com relação à medida gerada por esta mistura, é dada por

$$
f_{B E I}(y \mid \mu, \phi, \xi)= \begin{cases}\xi, & \text { se } y=c, \\ (1-\xi) f_{B}(y \mid \mu, \phi), & \text { se } y \in(0,1),\end{cases}
$$

em que $c=0$ ou $c=1,0<\xi<1$ representa a massa de probabilidade no ponto $c$ e $f_{B}(y \mid \mu, \phi)$ denota a densidade da variável beta (4.3). Diz-se que $Y$ tem distribuição beta inflacionada em zero e beta inflacionada em um, quando $c=0$ e $c=1$, respectivamente.

Denote por $y_{i j} \in(0,1]$ o valor da resposta longitudinal do $i$-ésimo indivíduo da amostra, em um instante $t_{j}$. Seja $\boldsymbol{y}_{i}=\left(y_{i 1}, \ldots, y_{i n_{i}}\right)^{\top}$ o vetor com as $n_{i}$ observações do indivíduo $i$. Condicional ao vetor de efeitos aleatórios $\boldsymbol{b}_{i}=\left(b_{0}, b_{1}\right)^{\top}$, assume-se que as respostas $y_{i j}$ são independentes e seguem distribuição beta inflacionada em um, com parâmetro $\mu_{i j} \in(0,1)$, parâmetro de precisão $\phi$ e parâmetro 
de mistura $\xi$. Os valores médios individuais são descritos por

$$
m_{i}(t)=E\left(Y_{i}(t) \mid \boldsymbol{b}_{i}\right)=\xi+(1-\xi) \mu_{i}(t)
$$

e a modelagem de $\mu_{i}(t)$ é feita através da ligação logito

$$
\log \left(\frac{\mu_{i}(t)}{1-\mu_{i}(t)}\right)=\boldsymbol{x}_{i}^{\top}(t) \boldsymbol{\beta}+\boldsymbol{z}_{i}^{\top}(t) \boldsymbol{b}_{i}
$$

em que $\boldsymbol{\beta}=\left(\beta_{1}, \ldots, \beta_{p}\right)$ é o vetor de efeitos fixos correspondente às variáveis explicativas $\boldsymbol{x}_{i}(t)=$ $\left(x_{i 1}(t), \ldots, x_{i p}(t)\right)^{\top}$ e $\boldsymbol{z}_{i}(t)=\left(z_{i 1}(t), z_{i 2}(t)\right)^{\top}$ é o vetor de variáveis explicativas associadas aos efeitos aleatórios. Assim como no modelo anterior, supõe-se que $\boldsymbol{b}_{i}$ tem distribuição normal multivariada, com vetor de média 0 e matriz de covariâncias $\boldsymbol{G}$.

\subsubsection{Objetivos inferenciais da modelagem proposta}

Uma grande dificuldade que envolve a modelagem de dados longitudinais de qualidade de vida é a omissão de dados. Nesses cenários, se o objetivo principal da investigação é estimar ou testar os efeitos de um conjunto de covariáveis nas respostas longitudinais, o uso dos modelos conjuntos pode ser útil para a obtenção de inferências válidas, pois através dessa abordagem é possível melhorar a precisão das estimativas e corrigir o viés decorrente da redução amostral (Molenberghs et al., 2014). Contudo, quando se trata de respostas longitudinais limitadas, a sua aplicação deve ser feita com cautela. É importante estar atento à interpretação dos parâmetros do modelo, bem como aos objetivos da modelagem.

Mais especificamente, na análise de dados longitudinais gaussianos o uso de um modelo linear com efeitos mistos preserva a interpretação populacional dos coeficientes de regressão $\boldsymbol{\beta}$. Isto ocorre porque sua marginalização resulta no seguinte valor esperado

$$
\begin{aligned}
E\left(Y_{i}(t)\right) & =E\left(E\left(Y_{i}(t) \mid \boldsymbol{b}_{i}\right)\right) \\
& =E\left(\boldsymbol{x}_{i}^{\top}(t) \boldsymbol{\beta}+z_{i}^{\top}(t) \boldsymbol{b}_{i}\right) \\
& =\boldsymbol{x}_{i}^{\top}(t) \boldsymbol{\beta} .
\end{aligned}
$$

Dessa forma, a taxa de variação média nas respostas individuais coincide com a taxa de variação da resposta média populacional. Por outro lado, nos modelos de regressão beta e beta inflacionado com efeitos mistos, descritos em (4.4) e (4.8), os coeficientes de regressão deixam de ter esta interpretação, 
uma vez que

$$
\operatorname{logito}\left(E\left(Y_{i}(t) \mid \boldsymbol{b}_{i}\right)\right)=\boldsymbol{x}_{i}^{\top}(t) \boldsymbol{\beta}+\boldsymbol{z}_{i}^{\top}(t) \boldsymbol{b}_{i} \quad \Longrightarrow \quad \operatorname{logito}\left(E\left(Y_{i j}\right)\right)=\boldsymbol{x}_{i}^{\top}(t) \boldsymbol{\beta} .
$$

A título de exemplo, no estudo do ICESP suponha que um parâmetro $\beta_{j}$ do modelo (4.4) ou (4.8) quantifique os efeitos de um tratamento quimioterápico na qualidade de vida de um paciente particular. A diferença entre os escores médios (na escala logito) de pacientes expostos e não expostos à quimioterapia, diz respeito somente aos indivíduos que compartilham as mesmas características específicas, representadas pelo mesmo conjunto de efeitos aleatórios. Neste contexto, se empregado dessa maneira, o coeficiente $\beta_{j}$ não é apropriado para caracterizar a evolução da população em estudo.

Tendo isso em vista, é importante esclarecer que os modelos longitudinais formulados nesta seção não são propostos com essa finalidade, mas com os objetivos inferenciais de (i) aprimorar a descrição dos tempos de vida observados; (ii) investigar a associação entre os processos longitudinal e de sobrevivência e (iii) obter predições para as probabilidades de sobrevivência individuais, considerando as informações dos perfis longitudinais de qualidade de vida dos pacientes.

\subsection{Estimação}

A estimação de máxima verossimilhança dos modelos conjuntos se baseia na maximização da função log-verossimilhança correspondente à distribuição conjunta dos tempos de sobrevivência e das respostas longitudinais, $\left\{T_{i}, \delta_{i}, \boldsymbol{Y}_{i}\right\}$, para todo $i=1, \ldots, n$. Essa distribuição é definida assumindo-se que o vetor de efeitos aleatórios é compartilhado pelos processos longitudinal e de sobrevivência e que condicional a $\boldsymbol{b}_{i}$ estas respostas são independentes, de tal forma que

$$
\begin{aligned}
p\left(T_{i}, \delta_{i}, \boldsymbol{y}_{i} \mid \boldsymbol{b}_{i}, \boldsymbol{\theta}\right) & =p\left(T_{i}, \delta_{i} \mid \boldsymbol{b}_{i}, \boldsymbol{\theta}_{t}\right) p\left(\boldsymbol{y}_{i} \mid \boldsymbol{b}_{i}, \boldsymbol{\theta}_{y}\right), \\
p\left(\boldsymbol{y}_{i} \mid \boldsymbol{b}_{i}, \boldsymbol{\theta}_{y}\right) & =\prod_{j=1}^{n_{i}} p\left(y_{i j} \mid \boldsymbol{b}_{i}, \boldsymbol{\theta}_{y}\right),
\end{aligned}
$$

$\operatorname{com} \boldsymbol{\theta}=\left(\boldsymbol{\theta}_{t}^{\top}, \boldsymbol{\theta}_{y}^{\top}, \boldsymbol{\theta}_{b}^{\top}\right)^{\top}$ denotando o vetor de parâmetros do modelo conjunto, composto pelos parâmetros $\boldsymbol{\theta}_{t}$ do submodelo de sobrevivência, $\boldsymbol{\theta}_{y}$ do submodelo longitudinal e $\boldsymbol{\theta}_{b}$ da matriz de covariâncias dos efeitos aleatórios. 
A função de verossimilhança de $\boldsymbol{\theta}$ para um indivíduo $i$, baseada em uma amostra constituída por $n$ observações independentes de $\left\{T_{i}, \delta_{i}, \boldsymbol{Y}_{i}\right\}$, é dada por

$$
\log p\left(T_{i}, \delta_{i}, \boldsymbol{y}_{i} \mid \boldsymbol{\theta}\right)=\log \int p\left(T_{i}, \delta_{i} \mid \boldsymbol{b}_{i}, \boldsymbol{\theta}_{t}, \boldsymbol{\beta}\right) \cdot\left[\prod_{j=1}^{n_{i}} p\left(y_{i j} \mid \boldsymbol{b}_{i}, \boldsymbol{\theta}_{y}\right)\right] \cdot p\left(\boldsymbol{b}_{i} \mid \boldsymbol{\theta}_{b}\right) d \boldsymbol{b}_{i} .
$$

O termo correspondente à componente de sobrevivência é expresso como

$$
p\left(T_{i}, \delta_{i} \mid \boldsymbol{b}_{i}, \boldsymbol{\theta}_{t}\right)=\left[h_{i}\left(T_{i} \mid \mathcal{M}_{i}(t), \boldsymbol{\theta}_{t}\right)\right]^{\delta_{i}} S_{i}\left(T_{i} \mid \mathcal{M}_{i}(t), \boldsymbol{\theta}_{t}\right)
$$

em que $h_{i}(\cdot)$ é a taxa de falha descrita em (4.1), $S_{i}(\cdot)$ é a função de sobrevivência (4.2), $p\left(y_{i j} \mid \boldsymbol{b}_{i}, \boldsymbol{\theta}_{y}\right)$ denota as densidades das distribuições beta (4.3) ou beta inflacionada (4.6) e $p\left(\boldsymbol{b}_{i} \mid \boldsymbol{\theta}_{b}\right)$ representa a densidade da distribuição normal multivariada.

Na estimação dos modelos conjuntos não é possível encontrar todos os elementos de $\hat{\boldsymbol{\theta}}$ através de fórmulas fechadas. Neste caso, para a maximização da log-verossimilhança

$$
\ell(\boldsymbol{\theta})=\sum_{i=1}^{n} \log p\left(T_{i}, \delta_{i}, \boldsymbol{y}_{i} \mid \boldsymbol{\theta}\right)
$$

a aproximação das estimativas geralmente é obtida de duas formas: (i) por meio do algoritmo EM (Dempster et al., 1977) e suas diversas variantes; ou (ii) através da maximização direta de $\ell(\boldsymbol{\theta})$. Dependendo da dimensão dos efeitos aleatórios ou do comportamento local da verossimilhança, esta pode ser uma tarefa computacionalmente intensiva. Com o objetivo de analisar qual a melhor estratégia para o tratamento numérico do processo de estimação, os aspectos teóricos e computacionais destes métodos são discutidos no Capítulo 5.

As integrais com respeito aos efeitos aleatórios e aos tempos de sobrevivência envolvidas na função de verossimilhança marginal (4.14) e na função (4.2) não possuem solução analítica. Neste trabalho, as aproximações numéricas são obtidas através das regras de quadratura de Gauss-Hermite (Quarteroni et al., 2010). Para a obtenção dos vetor escore e da matriz hessiana de $\ell(\boldsymbol{\theta})$ considera-se a diferenciação numérica por meio do método das diferenças finitas (Quarteroni et al., 2010). Estes procedimentos encontram-se descritos nas Seções A.1 e A.2, do Apêndice A. 


\subsection{Predições de qualidade de vida e de sobrevivência}

O desempenho do estimador (3.12) em amostras finitas depende das estimativas de máxima verossimilhança de $\boldsymbol{\theta}$, da predição do efeitos aleatórios e das novas medições longitudinais observadas para cada indivíduo. Neste sentido, a modelagem dos processos longitudinal e de sobrevivência é essencial para se garantir a precisão desses resultados. No caso das respostas longitudinais limitadas, o uso de distribuições com o mesmo suporte dos dados promove uma melhor descrição do seu comportamento, sobretudo em regiões próximas aos extremos do intervalo.

Esta seção mostra como os modelos conjuntos especificados neste capítulo podem aprimorar as predições das trajetórias longitudinais da qualidade de vida e das probabilidades de sobrevivência no estudo do ICESP. Para tanto, verifica-se de que forma as abordagens propostas atuam na predição dos efeitos aleatórios em situações nas quais (i) observações longitudinais unitárias ou próximas de zero são geradas pelos pacientes e em que (ii) a inferência para os efeitos aleatórios é feita sob o paradigma bayesiano, baseada na sua distribuição a posteriori (3.14).

Seja $\mathcal{D}_{n}=\left\{\left(T_{1}, \delta_{1}, \boldsymbol{y}_{1}\right), \ldots,\left(T_{n}, \delta_{n}, \boldsymbol{y}_{n}\right)\right\}$ uma amostra utilizada para o ajuste dos modelos conjuntos propostos. Considere um novo indivíduo $i$, que forneceu um conjunto de $n_{i}$ medidas longitudinais até um instante $t$ e para o qual deseja-se obter predições para as respostas de interesse. Suponha, sem perda de generalidade, que a sua última observação registrada seja igual a um e que as $n_{i}-1$ restantes estejam contidas no intervalo $(0,1)$, ou seja,

$$
\mathcal{Y}_{i}(t)=\left(y_{i 1}, y_{i 2}, \ldots, y_{i n_{i}-1}, 1\right)
$$

Na abordagem conjunta que utiliza o modelo de regressão beta com efeitos aleatórios, a seguinte distribuição a posteriori para $\boldsymbol{b}_{i}^{(t)}$ é gerada

$$
p\left(\boldsymbol{b}_{i}^{(t)} \mid T_{i}, \delta_{i}, \mathcal{Y}_{i}^{*}(t), \hat{\boldsymbol{\theta}}\right) \propto p\left(T_{i}, \delta_{i} \mid \boldsymbol{b}_{i}^{(t)}, \hat{\boldsymbol{\theta}}_{t}\right) \cdot\left[\prod_{j=1}^{n_{i}} f_{B}\left(y_{i j}^{*} \mid \boldsymbol{b}_{i}^{(t)}, \hat{\boldsymbol{\theta}}_{y}\right)\right] \cdot p\left(\boldsymbol{b}_{i}^{(t)} \mid \hat{\boldsymbol{\theta}}_{b}\right),
$$

em que $f_{B}\left(y \mid \boldsymbol{b}_{i}, \hat{\boldsymbol{\theta}}_{y}\right)$ representa a função densidade de probabilidade (4.3) e $\mathcal{Y}_{i}^{*}(t)$ é o vetor obtido mapeando-se o valor unitário de $\mathcal{Y}_{i}(t)$ no intervalo $(0,1)$. Note que todas as observações longitudinais do indivíduo $i$ são incorporadas na distribuição a posteriori (4.15), contribuindo para a predição dos efeitos aleatórios no instante $t$. Em particular, a observação da resposta unitária é inserida no modelo 
através do seu valor transformado.

Nos estudos longitudinais de qualidade de vida, uma proporção de escores máximos pode ser observada na amostra coletada. Muitas vezes essas medições são contaminadas por intervenções pontuais externas à evolução intrínseca do indivíduo, cujos efeitos não são explicados pelos modelos utilizados. Por exemplo, um paciente pode perceber sua saúde como perfeita após o controle de algum sintoma da doença, mas fornecer pontuações muito baixas nas coletas subsequentes devido à sua progressão. As ocorrências circunstanciais de escores máximos comprometem a predição das trajetórias médias de qualidade de vida, pois pode descrever a evolução do processo observado de maneira tendenciosa.

A influência desses tipos de observações pode ser atenuada na abordagem conjunta especificando-se as componentes longitudinais através da distribuição beta inflacionada em um. De fato, note que

$$
\begin{aligned}
p\left(\boldsymbol{b}_{i}^{(t)} \mid T_{i}, \delta_{i}, \boldsymbol{y}_{i}, \hat{\boldsymbol{\theta}}\right) & \propto p\left(T_{i}, \delta_{i} \mid \boldsymbol{b}_{i}^{(t)}, \hat{\boldsymbol{\theta}}_{t}\right) \cdot \prod_{j=1}^{n_{i}} f_{B E I}\left(y_{i j} \mid \boldsymbol{b}_{i}^{(t)}, \hat{\boldsymbol{\theta}}_{y}\right) \cdot p\left(\boldsymbol{b}_{i}^{(t)} \mid \hat{\boldsymbol{\theta}}_{b}\right) \\
& \propto p\left(T_{i}, \delta_{i} \mid \boldsymbol{b}_{i}^{(t)}, \hat{\boldsymbol{\theta}}_{t}\right) \cdot\left[\prod_{j=1}^{n_{i}-1}(1-\xi) f_{B}\left(y_{i j} \mid \boldsymbol{b}_{i}^{(t)}, \hat{\boldsymbol{\theta}}_{y}\right)\right] \cdot \xi \cdot p\left(\boldsymbol{b}_{i}^{(t)} \mid \hat{\boldsymbol{\theta}}_{b}\right) \\
& \propto p\left(T_{i}, \delta_{i} \mid \boldsymbol{b}_{i}^{(t)}, \hat{\boldsymbol{\theta}}_{t}\right) \cdot \prod_{j=1}^{n_{i}-1} f_{B}\left(y_{i j} \mid \boldsymbol{b}_{i}^{(t)}, \hat{\boldsymbol{\theta}}_{y}\right) \cdot p\left(\boldsymbol{b}_{i}^{(t)} \mid \hat{\boldsymbol{\theta}}_{b}\right)
\end{aligned}
$$

em que $f_{B E I}\left(y \mid \boldsymbol{b}_{i}, \hat{\boldsymbol{\theta}}_{y}\right)$ representa a função densidade de probabilidade (4.3). A informação contida na observação de valor unitário é inserida na distribuição a posteriori através do parâmetro $\xi$, que não compõe o núcleo da distribuição a posteriori (4.16). O valor dessa resposta, portanto, não é usado na predição dos efeitos aleatórios no instante $t$. Neste caso, a atualização de $\boldsymbol{b}_{i}^{(t)}$ é feita com base nas informações fornecidas por $\hat{\boldsymbol{\theta}}$ e nas medições longitudinais recolhidas nos instantes anteriores.

Outro aspecto inerente aos dados do ICESP é o prolongamento da sobrevida de pacientes em estado grave. É factível que o processo latente por trás desse fenômeno tenha relação com fatores que, apesar de não explicados pelas variáveis em estudo, geram uma percepção de bem-estar nos indivíduos, que é refletida nas observações através da proporção de pontuações máximas na amostra.

No modelo de regressão beta inflacionado, a probabilidade de observar um escore máximo na população em estudo, representada pelo parâmetro $\xi$, é usada para explicar a evolução da resposta média individual. Em particular, quando sucessivas observações longitudinais de um indivíduo são muito baixas, é esperado que as predições para a componente contínua do modelo tendam a zero. Neste caso, 
a estimativa do parâmetro $\hat{\xi}$ atua como um fator de correção na trajetória individual média, uma vez que

$$
\lim _{\hat{\mu}(t) \rightarrow 0} \hat{m}(t)=\lim _{\hat{\mu}(t) \rightarrow 0} \hat{\xi}+(1-\hat{\xi}) \cdot \hat{\mu}(t)=\hat{\xi}
$$

Esse aspecto do modelo inflacionado, relativo à descrição da média longitudinal inserida no modelo de sobrevivência, é utilizado para explicar o aumento da sobrevida em pacientes graves presentes nos dados do ICESP. Por exemplo, pode-se considerar na modelagem das trajetórias individuais os efeitos de importantes fatores que não são observados no estudo, como as assistências promovidas em cuidados paliativos. Embora os tratamentos com intenção paliativa tenham o objetivo de gerar o bem-estar dos pacientes através da prevenção e alívio da dor e demais sintomas físicos e psicológicos, é possível que essas práticas retardem indiretamente o tempo até o óbito.

\subsection{Erros padrão bootstrap para as predições de sobrevivência}

A obtenção dos erros padrão e dos intervalos de confiança para as predições de sobrevivência é uma tarefa bastante complexa, uma vez que deve-se levar em conta a variabilidade do estimador de máxima verossimilhança de $\boldsymbol{\theta}$ e do estimador empírico de Bayes para $\boldsymbol{b}_{i}$. Uma solução computacional para este problema é proposta em Rizopoulos et al. (2009) através de um esquema de simulação híbrido derivado a partir de propriedades assintóticas do estimador de máxima verossimilhança e algoritmos bayesianos. Nesta seção, apresenta-se uma abordagem frequentista para a geração de erros padrão para as predições, baseada na reamostragem do tipo bootstrap balanceada dos dados originais.

O bootstrap é uma técnica de reamostragem introduzida por Efron (1979), que permite aproximar a distribuição amostral de uma estatística de interesse pela distribuição empírica dos dados, baseada em uma amostra de tamanho finito. A reamostragem é feita com reposição (i) da distribuição da qual os dados são obtidos (bootstrap paramétrico) ou (ii) baseada na amostra original (bootstrap não paramétrico). Neste trabalho adota-se a abordagem não paramétrica.

As respostas longitudinais e de sobrevivência, quando coletadas conjuntamente, possuem características que não são preservadas em uma reamostragem uniforme, na qual todos os elementos têm a mesma probabilidade de serem selecionados. Por se tratar de dados agrupados, esse processo deve ser realizado em nível individual e não em termos das observações. Dessa forma, é possível respeitar a 
estrutura de dependência das medidas repetidas. Além disso, é importante que outros aspectos sejam levados em conta:

i) Por causa dos abandonos ao longo do acompanhamento, alguns indivíduos possuem mais observações longitudinais do que outros. Para que a reamostragem mantenha esta característica dos dados é necessário que as amostras bootstrap apresentem, em média, o mesmo número de observações individuais da amostra original. Note que como estas variáveis são dependentes do tempo, e são coletadas conjuntamente com os tempos de sobrevivência, um procedimento realizado de forma inadequada pode gerar amostras bootstrap nas quais o tempo de vida médio é muito diferente do observado originalmente.

ii) Quando as variáveis longitudinais são limitadas no intervalo $(0,1]$ existe uma proporção de observações com valores iguais a um nos dados coletados. O esquema de reamostragem deve garantir que amostras sorteadas possuam, em média, a mesma quantidade desses pontos extremos.

iii) Com relação aos tempos de sobrevivência, é importante que a proporção de censura da amostra original seja preservada. Além disso, deve-se evitar os casos em que todas as amostras sorteadas sejam compostas por observações censuradas.

Para que a proporção de censura seja mantida no processo de reamostragem, considera-se a obtenção de amostras bootstrap separadamente, em subamostras de observações censuradas e não censuradas dos dados originais. Em cada um destes conjuntos, as estruturas discutidas em (i) e (ii) são preservadas através da reamostragem balanceada. A ideia desta técnica, descrita em Gleason (1988), é reamostrar os dados originais assegurando que cada indivíduo seja selecionado, em média, o mesmo número de vezes nas amostras bootstrap sorteadas. Este procedimento encontra-se esquematizado no algoritmo a seguir.

\subsubsection{Boostrap balanceado para dados longitudinais e de sobrevivência}

Denote por $\mathcal{D}_{n}=\left\{\mathcal{D}_{n_{1}}, \mathcal{D}_{n_{2}}\right\}$ o conjunto de dados original, em que $\mathcal{D}_{n_{1}}$ e $\mathcal{D}_{n_{2}}$ são os subconjuntos de $\mathcal{D}_{n}$ contendo as $n_{1}$ unidades amostrais com observações censuradas e as $n_{2}$ unidades amostrais com observações não censuradas, respectivamente. 
1. Para cada subamostra $\mathcal{D}_{n_{k}}(k=1,2)$ :

a) Concatene $B$ cópias das observações de cada unidade amostral,

$$
\underbrace{\left(T_{1}^{(k)}, \delta_{1}^{(k)}, \boldsymbol{y}_{1}^{(k)}\right), \ldots,\left(T_{1}^{(k)}, \delta_{1}^{(k)}, \boldsymbol{y}_{1}^{(k)}\right)}_{B \text { vezes }}, \ldots, \underbrace{\left(T_{n}^{(j)}, \delta_{n}, \boldsymbol{y}_{n}^{(k)}\right), \ldots,\left(T_{n}^{(k)}, \delta_{n}^{(k)}, \boldsymbol{y}_{n}^{(k)}\right)}_{B \text { vezes }},
$$

em que $\left(T_{i}^{(k)}, \delta_{i}^{(k)}, \boldsymbol{y}_{i}^{(k)}\right)$ denota as observações do $i$-ésimo indivíduo da subamostra $\mathcal{D}_{m_{k}}$.

b) Permute os $n \times B$ valores da sequência obtida e selecione $B$ amostras de tamanho $n$,

$$
\underbrace{\left(T_{11}^{(k)}, \delta_{11}^{(k)}, \boldsymbol{y}_{11}^{(k)}\right), \ldots,\left(T_{n 1}^{(k)}, \delta_{n 1}^{(k)}, \boldsymbol{y}_{n 1}^{(k)}\right)}_{B_{1}^{(k)}}, \ldots, \underbrace{\left(T_{1 B}^{(k)}, \delta_{1 B}^{(k)}, \boldsymbol{y}_{1 B}^{(k)}\right), \ldots,\left(T_{n B}^{(k)}, \delta_{n B}^{(k)}, \boldsymbol{y}_{n B}^{(k)}\right)}_{B_{B}^{(k)}},
$$

em que $\left(T_{r s}^{(k)}, \delta_{r s}^{(k)}, \boldsymbol{y}_{r s}^{(k)}\right)$ denota o $r$-ésimo elemento da amostra bootstrap $B_{s}^{(k)}$.

2. As amostras bootstrap balanceadas são dadas por

$$
B_{b}=\left(B_{b}^{(1)}, B_{b}^{(2)}\right), \quad b=1, \ldots, B
$$

\section{Estimativas bootstrap para os erros padrão das predições de sobrevivência}

1. Para cada amostra bootstrap, obtenha as réplicas correspondentes para as estimativas de máxima verossimilhança de $\boldsymbol{\theta}$ e para as predições dos efeitos aleatórios no instante $t$, denotados respectivamente por $\boldsymbol{\theta}^{(b)}$ e $\boldsymbol{b}_{i}^{(b)}$.

2. Para cada $b=1, \ldots, B$, calcule

$$
\pi_{i}^{(b)}(u \mid t)=\frac{S_{i}\left(u \mid \mathcal{M}_{i}\left(u, \boldsymbol{b}_{i}^{(b)}, \boldsymbol{\theta}^{(b)}\right), \boldsymbol{\theta}^{(b)}\right)}{S_{i}\left(t \mid \mathcal{M}_{i}\left(t, \boldsymbol{b}_{i}^{(b)}, \boldsymbol{\theta}^{(b)}\right), \boldsymbol{\theta}^{(b)}\right)}
$$

3. Utilize as realizações $\pi_{i}^{(b)}(u \mid t)$ para obter as estimativas pontuais

$$
\hat{\pi}_{i}(u \mid t)=\frac{1}{B} \sum_{i=1}^{n} \pi_{i}^{(b)}(u \mid t)
$$


4. A estimativa para o erro padrão de $\hat{\pi}_{i}(u \mid t)$ é dada pelo desvio padrão amostral das realizações $\left\{\pi_{i}^{(b)}(u \mid t) ; l=1, \ldots, B\right\}$. 


\section{Capítulo 5}

\section{Aspectos computacionais do processo de estima- ção dos modelos conjuntos}

Os modelos conjuntos para dados longitudinais e tempos de sobrevivência têm um grande potencial de aplicação em análises estatísticas. As diversas extensões propostas na literatura explicam aspectos dos processos observados que permitem realizar a estimação de efeitos complexos (Rizopoulos, 2012), investigar a dinâmica entre a sua associação ou obter predições com maior precisão. Em muitas situações, no entanto, os ganhos inferenciais gerados por meio de modelagens mais sofisticadas são minimizados diante das dificuldades enfrentadas na etapa de estimação.

Considerando o paradigma frequentista, nem sempre é possível maximizar a função de verossimilhança de forma analítica. Nesses casos, é necessário utilizar procedimentos iterativos que, embora gerem aproximações para as estimativas, entregam precisão suficiente para fins inferenciais e preditivos. Em geral, esse é um problema desafiador do ponto de vista computacional, pois os algoritmos empregados na estimação podem apresentar graves falhas de convergência ou progredir muito lentamente até que uma solução seja encontrada com a precisão desejada. Essas dificuldades se somam ao esforço exigido no tratamento numérico da verossimilhança, que geralmente envolvem a integração de funções de várias variáveis.

Diversas ferramentas estatísticas e computacionais têm sido desenvolvidas para o ajuste de modelos conjuntos nas últimas décadas. Grande parte dessas propostas se concentra no ganho de eficiência: trabalhos como Lin et al. (2002) e Philipson et al. (2020) utilizam o algoritmo EM - Monte Carlo para otimizar o processamento de integrais multidimensionais; em Rizopoulos (2012), o algoritmo EM é combinado a procedimentos mais rápidos com o objetivo de acelerar a convergência do processo iterativo; e na 
estimação bayesiana, os métodos sequenciais de Monte Carlo são considerados em Alvares et al. (2020) como alternativa mais eficiente aos métodos de Monte Carlo via cadeias de Markov. Embora a velocidade de processamento seja uma medida de desempenho priorizada em muitos casos, uma boa prática computacional exige que outros atributos sejam levados em conta, sobretudo em problemas de maior complexidade.

Este capítulo explora os aspectos teóricos e as propriedades computacionais de algoritmos usados no ajuste de modelos conjuntos. As discussões apresentadas retratam o problema de estimação sob a ótica da computação científica, e fornecem meios para uma seleção apropriada dos procedimentos numéricos. Para isso, os métodos iterativos empregados em análises frequentistas são avaliados de forma padronizada, baseado nas seguintes propriedades recomendadas em Nocedal e Wright (2006):

1. Robustez: A robustez de um algoritmo numérico está associada à sua capacidade de fornecer soluções consistentes em diversas instâncias que o método se propõe a resolver, para todas as escolhas razoáveis de pontos iniciais. Isto só é possível quando existem garantias teóricas para sua convergência (caso contrário, estes procedimentos são chamados de heurísticas).

2. Precisão e estabilidade: Um método é considerado preciso se suas aproximações possuem um grau de acurácia adequado ao escopo da aplicação, de acordo com alguma tolerância prefixada. Para isto, é importante que o procedimento iterativo apresente estabilidade, ou seja, que sua execução não amplifique os erros de aproximações, causando grandes desvios na resposta final.

3. Eficiência: Um método iterativo é eficiente se for capaz de gerar boas soluções aproximadas, sem exigir muito tempo de processamento ou espaço em memória para ser executado.

4. Facilidade de implementação: O ideal é que os algoritmos iterativos empregados na estimação de um modelo sejam de fácil implementação. Por essa razão, o uso de procedimentos que exijam o uso rotinas ou configurações muito complicadas para o seu funcionamento deve ser ponderado de acordo com a necessidade e objetivos de cada aplicação. 
Nem sempre é possível encontrar uma classe de procedimentos que contemple, de forma simultânea, todas as propriedades enumeradas. A alternativa, muitas vezes, é fazer um balanço entre os critérios considerando as especificidades de cada problema, como exemplifica-se a seguir:

a) Quando se trata da formulação de novas extensões de modelos, a capacidade do algoritmo de gerar resultados consistentes, a partir de escolhas razoáveis de pontos iniciais, pode ser mais importante do que a rapidez e o esforço computacional;

b) Se a modelagem conjunta é feita com propósitos preditivos é importante que a robustez não esteja associada a grandes tempos de processamento. De outro modo, a validação das predições através de técnicas baseadas em reamostragem pode ter um custo proibitivo;

c) Quando o consumo de memória for um problema, como em análises de grandes conjuntos de dados, é recomendável priorizar procedimentos com menor demanda de armazenamento, mesmo que isso provoque uma redução na velocidade de convergência;

d) O uso do paradigma bayesiano em análises conjuntas vem ganhando grande adesão por parte dos pesquisadores devido à sua simplicidade de implementação, que não precisa do cálculo de integrais nos efeitos aleatórios. Por outro lado, um aspecto negativo da abordagem é que, dependendo das características do problema, os algoritmos bayesianos exigem maiores tempos de execução. Sendo assim, se a análise dos dados demandar eficiência, pode ser vantajoso investir em uma implementação mais sofisticada de procedimentos frequentistas.

Com o objetivo de contribuir com esse tipo de avaliação, uma análise computacional de métodos tipicamente empregados na estimação de modelos conjuntos é apresentada nas próximas seções. Para uma melhor compreensão de questões relacionadas à convergência, que são mencionadas ao longo do presente capítulo, a discussão é iniciada na Seção 5.1, na qual são introduzidos alguns conceitos de análise numérica. As Seções 5.2, 5.3 e 5.4 exploram aspectos metodológicos do algoritmo EM, de suas extensões para o ganho de eficiência e dos métodos de busca linear, enfatizando as suas principais vantagens e desvantagens computacionais na estimação de máxima verossimilhança. 


\subsection{Métodos iterativos e ordem de convergência}

Em abordagens frequentistas, os principais algoritmos usados no processo de estimação são iterativos. Nesses métodos, um conjunto de instruções é executado de modo sistemático até a obtenção de uma aproximação razoável da solução exata, contida no espaço paramétrico $\Theta \subset \mathbb{R}^{p}$. Os procedimentos são inicializados em um ponto $\boldsymbol{\theta}_{0}$ (chute inicial), e atualizados através de uma relação de recorrência da forma

$$
\boldsymbol{\theta}_{k+1}=\Psi\left(\boldsymbol{\theta}_{k}\right), \quad k=0,1,2, \ldots
$$

em que $\Psi(\cdot)$ é uma função especificada de acordo com a método utilizado. Assim, a repetição de (5.1) gera uma sequência de iterandos

$$
\left\{\boldsymbol{\theta}_{k}\right\}_{k \geq 0}=\left\{\boldsymbol{\theta}_{0}, \boldsymbol{\theta}_{1}, \boldsymbol{\theta}_{2}, \ldots\right\}
$$

e termina quando um ponto estacionário $\theta^{*}$ é identificado com o grau de precisão desejado. Para que o processo iterativo seja encerrado em uma situação de convergência, é preciso estabelecer um critério de parada. Isto pode ser feito baseado no erro absoluto da $k$-ésima aproximação, que é definido como

$$
\varepsilon_{k}=\left\|\boldsymbol{\theta}_{k}-\boldsymbol{\theta}^{*}\right\|
$$

em que $\|\cdot\|$ denota, por exemplo, a norma euclidiana de um vetor de um subespaço do $\mathbb{R}^{n}$.

Uma importante medida de desempenho na avaliação dos métodos iterativos é a ordem de convergência. A partir de diferentes definições para essa métrica, diversas taxas de convergência podem ser identificadas nos procedimentos numéricos. No entanto, para as análises discutidas neste capítulo, a seguinte classificação é suficiente.

Definição 1 Seja $\left\{\boldsymbol{\theta}_{k}\right\}_{k \geq 0}$ uma sequência de iterandos obtida através de um método da forma (5.1). Suponha que essas aproximações sejam convergentes para um ponto estacionário $\boldsymbol{\theta}^{*} \in \Theta$.

i) A sequência $\left\{\boldsymbol{\theta}_{k}\right\}_{k \geq 0}$ converge com ordem linear para $\boldsymbol{\theta}^{*}$ se existe uma constante $C \in(0,1)$ tal que

$$
\lim _{k \rightarrow \infty} \frac{\varepsilon_{k+1}}{\varepsilon_{k}}=C .
$$


ii) Nos casos em que $C=0$ e $C=1 \mathrm{em}$ (5.4), a sequência $\left\{\boldsymbol{\theta}_{k}\right\}_{k \geq 0}$ converge com ordem superlinear e sublinear para $\boldsymbol{\theta}^{*}$, respectivamente.

iii) A sequência $\left\{\boldsymbol{\theta}_{k}\right\}_{k \geq 0}$ converge com ordem $q$ para $\boldsymbol{\theta}^{*}$ se existem constantes $C \in(0, \infty)$ e $q>1$ tais que

$$
\lim _{k \rightarrow \infty} \frac{\varepsilon_{k+1}}{\varepsilon_{k}^{q}}=C
$$

Adicionalmente, da existência dos limites anteriores, é possível mostrar que existe uma constante $M \in \mathbb{N}$ suficientemente grande, tal que a aproximação

$$
\left\|\boldsymbol{\theta}_{k+1}-\boldsymbol{\theta}^{*}\right\| \approx C\left\|\boldsymbol{\theta}_{k}-\boldsymbol{\theta}^{*}\right\|^{q}, \quad \forall k>M
$$

é válida para cada uma das ordens de convergência apresentadas.

Os métodos de convergência linear geram, a cada iteração, a mesma redução no erro absoluto. Dependendo da complexidade da função analisada, este pode ser um comportamento muito lento, principalmente nas regiões próximas ao ponto $\boldsymbol{\theta}^{*}$. Nos algoritmos com ordem de convergência 2 , conforme a sequência de iteradas se aproxima de um ponto estacionário, os erros absolutos se reduzem a uma taxa quadrática. Esse aspecto permite observar convergência nas primeiras iterações, desde que sejam fornecidos bons chutes iniciais. Já os métodos com ordem de convergência superlinear, embora mais lentos que o anterior, oferecem eficiência, pois também reduzem os erros absolutos de iterações subsequentes.

\subsection{Algoritmo EM}

O algoritmo de EM, proposto por Dempster et al. (1977), é um método iterativo usado na resolução de problemas com dados incompletos. Este procedimento tem se mostrado bastante popular na estimação de máxima verossimilhança dos modelos conjuntos, nos quais os efeitos aleatórios são tratados como dados omissos. Em poucas palavras, a ideia desta metodologia é que a log-verossimilhança relativa às observações incompletas, em geral, é mais simples de se maximizar. 
Denote por $\boldsymbol{Y}$ o vetor contendo as respostas longitudinais completas, que é composta por uma parte observada $\boldsymbol{Y}^{o}$ e uma parte omissa $\boldsymbol{Y}^{m}$. O objetivo é estimar os parâmetros correspondentes aos dados completos, usando apenas as informações observadas. Assim, a cada iteração, o método percorre dois passos:

- Etapa E: Para lidar com as observações incompletas, calcula-se o valor esperado da log-verossimilhança, dada as respostas completas:

$$
\begin{aligned}
Q\left(\boldsymbol{\theta} \mid \boldsymbol{\theta}_{k}\right) & =\mathrm{E}\left\{\log p(\boldsymbol{y} \mid \boldsymbol{\theta}) \mid \boldsymbol{y}^{o}, \boldsymbol{\theta}_{k}\right\} \\
& =\int \log p\left(\boldsymbol{y}^{\boldsymbol{o}}, \boldsymbol{y}^{m} \mid \boldsymbol{\theta}\right) p\left(\boldsymbol{y}^{m} \mid \boldsymbol{y}^{\boldsymbol{o}}, \boldsymbol{\theta}_{k}\right) d \boldsymbol{y}^{m}
\end{aligned}
$$

Na aplicação do algoritmo EM ao contexto dos modelos conjuntos, os efeitos aleatórios são tratados como omissões. Dessa forma, o valor esperado da log-verossimilhança completa é dado por

$$
Q\left(\boldsymbol{\theta} \mid \boldsymbol{\theta}_{k}\right)=\sum_{i} \int \log p\left(T_{i}, \delta_{i}, \boldsymbol{y}_{i}, \boldsymbol{b}_{i} \mid \boldsymbol{\theta}\right) p\left(\boldsymbol{b}_{i} \mid \boldsymbol{\theta}\right) d \boldsymbol{b}_{i},
$$

Para a avaliação de (5.8), procedimentos de integração numérica devem ser empregados, como regras de quadratura gaussiana ou técnicas de amostragem de Monte Carlo.

- Etapa M: A atualização da sequência de iterandos é dada pela maximização

$$
\boldsymbol{\theta}_{k+1}=\underset{\boldsymbol{\theta}}{\operatorname{argmax}} Q\left(\boldsymbol{\theta} \mid \boldsymbol{\theta}_{k}\right)
$$

Em Rizopoulos (2012), mostra-se que para os modelos conjuntos padrão, nos quais a componente longitudinal é especificada por um modelo linear com efeitos mistos e a componente de sobrevivência é definida pelo modelo de taxa de falhas proporcionais, algumas das expressões exigidas para o cálculo de (5.9) apresentam fórmulas fechadas, tornando esta etapa do processo iterativo menos custosa.

O procedimento é encerrado quando for evidente que (i) mais iterações não mudarão substancialmente a aproximação para o estimador de máxima verossimilhança, ou (ii) não mudarão substancialmente o valor da função a ser maximizada. 
Uma das principais vantagens computacionais do algoritmo EM é o comportamento monótono da sua sequência de aproximações $\left\{\boldsymbol{\theta}_{k}\right\}_{k \geq 0}$. Em Dempster et al. (1977), mostra-se que cada atualização na etapa de maximização promove um aumento na função log-verossimilhança, isto é

$$
\ell\left(\boldsymbol{\theta}_{k+1}\right) \geq \ell\left(\boldsymbol{\theta}_{k}\right), \quad \forall k=0,1,2, \ldots
$$

Em trabalhos como Yang et al. (2016) e Xu et al. (2014), a propriedade (5.10) é considerada uma condição suficiente para a estabilidade numérica. Isso não é necessariamente válido quando se trata da estimação de modelos conjuntos. Nesse caso, a etapa de maximização não pode ser resolvida de modo explícito, o que torna o procedimento duplamente iterativo. Consequentemente, as operações envolvidas na sua execução estão sujeitas a imprecisões numéricas que podem gerar a instabilidade do processo, independente do comportamento monótono de $\left\{\ell\left(\boldsymbol{\theta}_{k}\right)\right\}_{k \geq 0}$.

Na verdade, a monotonicidade em (5.10) garante apenas que a solução seja procurada em alguma direção de crescimento da verossimilhança. Este fato, visto isoladamente, não implica na convergência de $\left\{\boldsymbol{\theta}_{k}\right\}_{k \geq 0}$. Dependendo do valor inicial e de condições de regularidade impostas sobre $\ell(\boldsymbol{\theta})$ e $Q\left(\boldsymbol{\theta} \mid \boldsymbol{\theta}_{k}\right)$, as aproximações $\left\{\boldsymbol{\theta}_{k}\right\}_{k \geq 0}$ poderão convergir para um máximo global, local ou para um ponto de sela (Wu, 1983; Boyles, 1983; Neath et al., 2013). Assim, é importante ter em conta que não existe um teorema geral de convergência para o algoritmo EM (Gupta e Chen, 2011). Por esta razão, ao se aplicar essa classe de procedimentos no ajuste de novas extensões de modelos conjuntos, a validade das soluções deve ser verificada em cenários controlados analisando-se a robustez dos resultados obtidos diante de diferentes valores iniciais, em conjuntos de dados artificiais.

\subsection{Extensões do algoritmo EM}

Uma das desvantagens computacionais do algoritmo EM é a sua ordem de convergência linear. Isso significa que os elementos da sequência $\left\{\boldsymbol{\theta}^{(k)}\right\}_{k \geq 0}$ são atualizados de maneira mais lenta em uma vizinhança do ponto de máximo, resultando em um grande número de iterações até o fim do processo. Algumas estratégias para o ganho de eficiência geram extensões do algoritmo voltadas para a redução do custo de avaliação das integrais e para o uso de métodos híbridos para a aceleração da convergência. 
Quando as integrais envolvidas em (5.8) são multidimensionais e não possuem solução analítica, a execução do método iterativo pode ter um custo computacional proibitivo. Uma modificação popular do algoritmo EM, proposta por Wei e Tanner (1990), é obtida quando a esperança na Etapa E é aproximada por técnicas de amostragem de Monte Carlo. Esses procedimentos, conhecidos como Algoritmos EM Monte Carlo, podem ser uma alternativa eficiente na estimação de máxima verossimilhança de modelos conjuntos, principalmente quando o vetor de efeitos aleatórios é de alta dimensão. Antes de considerálos, no entanto, é importante ter ciência da sua robustez em situações práticas.

O estudo da convergência dos algoritmos EM - Monte Carlo é mais complicado quando comparado aos EM tradicionais (uma discussão acessível sobre os principais resultados de convergência destes procedimentos pode ser encontrada em Neath et al. (2013)). Uma questão importante nesse sentido é a quantidade de pontos necessários para a simulação Monte Carlo. Seja $\boldsymbol{b}^{(1)}, \ldots, \boldsymbol{b}^{(m)}$ uma amostra da distribuição $p(\boldsymbol{b} \mid Z, \delta, \boldsymbol{y})$. Então, a aproximação estocástica para (5.8) é dada por

$$
Q_{m}\left(\boldsymbol{\theta} \mid \boldsymbol{\theta}_{k}\right)=\frac{1}{m} \sum_{r=1}^{m} \log p\left(Z, \delta, \boldsymbol{y}, \boldsymbol{b}^{(r)} \mid \boldsymbol{\theta}\right) .
$$

Para que a convergência seja atingida, o tamanho da amostra Monte Carlo deve aumentar com o avanço das iterações. Esse é um comportamento plausível, pois para pontos mais afastados da estimativa de máxima verossimilhança, uma menor precisão é exigida das aproximações. À medida que os iterandos atingem convergência, é razoável que a função a ser maximizada seja calculada com maior acurácia. Sendo assim, em cada situação na qual esse procedimento é aplicado, é necessário se fazer um balanço sobre o esforço computacional desse método de integração comparado aos métodos determinísticos, como as regras de quadratura. Além disso, embora se saiba que o valor de $m$ é uma função crescente do número de iterações, não existem resultados que esclareçam de que modo esta relação é dada. Dessa forma, é comum que os números de amostras Monte Carlo sejam incrementados empiricamente.

Um outro problema prático da convergência dos algoritmos EM - Monte Carlo está relacionado às regras de parada. Os critérios usuais empregados na sua versão com integrais determinísticas podem fazer com que o procedimento seja encerrado antes do que deveria. É possível, por exemplo, que em uma das amostras geradas para a aproximação, o erro seja pequeno o suficiente a ponto de sinalizar a convergência do processo. Algumas soluções para este problema são: (i) encerrar o procedimento 
quando a convergência for atestada pelos critérios após três iterações consecutivas (Booth et al., 2001); (ii) terminar o algoritmo quando $\left|\ell\left(\boldsymbol{\theta}_{k}\right)-\ell\left(\boldsymbol{\theta}_{k-1}\right)\right|$ for estocasticamente pequeno (Chan e Ledolter, 1995); e (iii) nesta mesma direção, finalizar o processo quando um limitante superior assintótico para $\left|Q\left(\boldsymbol{\theta}_{k} \mid \boldsymbol{\theta}_{k-1}\right)-Q\left(\boldsymbol{\theta}_{k-1} \mid \boldsymbol{\theta}_{k-1}\right)\right|$ for menor do que uma tolerância prefixada (Cao et al., 2005).

Observe que, apesar de reduzirem o custo da etapa E, os algoritmos EM - Monte Carlo ainda convergem à uma taxa linear. Por essa razão, em uma abordagem tratada na literatura, a aceleração da convergência é buscada por meio de métodos híbridos. As iterações são inicializadas com o algoritmo EM, e o restante das aproximações são obtidas através de procedimentos com ordens de convergência maiores, como os métodos de Newton ou quase-Newton (Rizopoulos, 2012). Vale ressaltar que, embora eficiente em muitas situações, esta estratégia é uma heurística. Isso significa que as soluções obtidas por esses algoritmos podem ser geradas em tempos relativamente menores, sem que haja garantias sobre a sua qualidade.

\subsection{Métodos de busca linear}

Em termos matemáticos, otimização refere-se à minimização ou à maximização de funções que podem estar sujeitas ou não à restrições em seus parâmetros. Ao longo das próximas seções utiliza-se a expressão função objetivo para se referir às funções que deseja-se otimizar. Em abordagens frequentistas, a estimação de máxima verossimilhança é tratada como um problema de otimização determinístico e resolvida através de métodos desenvolvidos para funções não lineares nas seus parâmetros. Os algoritmos usados para esse fim são procedimentos iterativos que são inicializados um ponto $\boldsymbol{\theta}_{0}$, geram uma sequência de iterandos $\left\{\boldsymbol{\theta}^{(k)}\right\}_{k \geq 0}$ e terminam quando uma solução é encontrada com a precisão desejada.

Uma estratégia para a atualização de um ponto $\boldsymbol{\theta}_{k}$ para um novo valor $\boldsymbol{\theta}_{k+1}$ nos métodos de otimização não linear é a chamada busca linear. Para ilustrar o funcionamento dessa classe de algoritmos, a descrição de seus passos é feita a seguir em termos da maximização da log-verossimilhança $\ell(\theta)$. 


\subsubsection{Algoritmos de busca linear}

Determine um valor $\boldsymbol{\theta}_{0}$ para ser utilizado como uma aproximação inicial para a estimativa de interesse

$\hat{\boldsymbol{\theta}}$. Após a inicialização da sequência de iterandos, na $k$-ésima iteração faça:

1. Encontre uma direção de crescimento da função objetivo (direção ascendente), a qual é denotada por $\boldsymbol{p}_{k}$;

2. Calcule a distância $v_{k}$ a se mover nesta direção, a partir do ponto $\boldsymbol{\theta}_{k}$;

3. Obtenha um novo valor para a sequência de aproximações $\left\{\boldsymbol{\theta}_{1}, \ldots, \boldsymbol{\theta}_{k}\right\}$ por meio da regra seguinte regra de atualização

$$
\boldsymbol{\theta}_{k+1}=\boldsymbol{\theta}_{k}+v_{k} \boldsymbol{p}_{k}
$$

4. Encerre o procedimento quando um ponto estacionário $\boldsymbol{\theta}^{*}$ for encontrado com a precisão desejada. Uma estratégia, por exemplo, é verificar se o gradiente da função objetivo é pequeno (segundo alguma norma) naquela iteração, de acordo uma tolerância prefixada $\epsilon$, ou seja, se

$$
|| \nabla \ell\left(\boldsymbol{\theta}_{k}\right) \|<\epsilon .
$$

As direções ascendentes dos métodos de busca linear são obtidas como solução do seguinte sistema linear

$$
\boldsymbol{B}_{k} \boldsymbol{p}_{k}=\nabla \ell_{k}, \quad k=0,1,2, \ldots,
$$

em que $\boldsymbol{B}_{k}$ é uma matriz simétrica e não singular e $\nabla \ell_{k}$ é o gradiente da log-verossimilhança marginal avaliado no ponto $\boldsymbol{\theta}_{k}$. Diferentes algoritmos que pertencem a essa classe são estabelecidos de acordo com a especificação de $\boldsymbol{B}_{k}$. Em geral, consideram-se aqueles que não utilizam apenas as direções delineadas pelo vetor gradiente, mas também incorporam informações de curvatura local. Isso confere ao procedimento maior precisão na execução dessa etapa.

Quando a matriz $\boldsymbol{B}_{k}$ é a matriz hessiana de $\ell\left(\boldsymbol{\theta}_{k}\right)$, denotada por $\mathbf{H}_{k}$, o algoritmo obtido é conhecido como método de Newton. Nos casos em que essas matrizes não estão disponíveis ou são obtidas a um custo computacional alto é possível aproximá-las utilizando a informação de primeira ordem fornecida pelo vetor gradiente. Essa classe de procedimentos é chamada de métodos quase-Newton. 
A distância a se mover ao longo de $p_{k}$ é definda, a cada iteração, pela resolução do problema unidimensional

$$
\underset{v_{k} \geq 0}{\operatorname{Maximizar}} \ell\left(\boldsymbol{\theta}_{k}+v_{k} \boldsymbol{p}_{k}\right), \quad k=0,1,2, \ldots,
$$

em que $v_{k}$ é um escalar positivo chamado tamanho de passo. Encontrar $v_{k}$ de maneira exata pode ser muito custoso em um processo iterativo. Uma estratégia para reduzir esse esforço computacional é gerar um número limitado de tentativas de tamanho de passo até se obter um valor que aproxima a solução do problema (5.13). Algumas técnicas para a execução dessa etapa, como o backtracking, são descritas em Gill et al. (2019) e Nocedal e Wright (2006), entre outros. Para a leitura deste trabalho, é suficiente compreender que o funcionamento desse passo depende do comportamento da função objetivo e portanto pode apresentar falhas em regiões de alta sensibilidade (que oscilam muito mediante a pequenas perturbações de seus parâmetros). Esse tipo de problema recorrente em abordagens numéricas é discutido no Capítulo 6, em um contexto de modelagem conjunta.

Os métodos de Newton e quase-Newton são procedimentos que possuem garantias teóricas de convergência ${ }^{1}$, e são estáveis se inicializados com bons chutes iniciais. Por serem de fácil utilização e implementação, estes algoritmos encontram-se disponíveis em diversas linguagens de programação estatística. Assim, empregá-los em procedimentos inferenciais baseados na otimização de algum critério, como a estimação de máxima verossimilhança, é uma maneira segura de se obter aproximações numéricas para as estimativas. De um modo geral, a principal diferença computacional entre esses algoritmos está na sua taxa de convergência e demanda por armazenamento. Nos métodos de Newton, a ordem de convergência é quadrática. Esta velocidade pode entregar um alto desempenho, especialmente em regiões próximas ao ponto de máximo. No entanto, dependendo do número de parâmetros do modelo, será necessário o cálculo e a inversão de matrizes de alta dimensão. Nesses casos, os métodos quase-Newton podem ser uma alternativa mais viável. Ao medir as mudanças no gradiente de uma iteração para outra, esses procedimentos constroem um modelo da função objetivo bom o suficiente para produzir convergência superlinear, fornecendo um equilíbrio entre rapidez e baixo consumo de espaço em memória.

Finalmente, os métodos discutidos nesta seção são procedimentos de otimização irrestrita, que minimizam ou maximizam funções que não possuem restrições impostas nas variáveis. Nos casos em que

\footnotetext{
${ }^{1}$ Os métodos descritos possuem resultados conhecidos de convergência local, e suas condições são razoáveis de serem verificadas em situações práticas, pois estão condicionadas à convexidade da função objetivo, ao comportamento das matrizes hessianas no ponto de máximo, bem como da sequência de iterandos. Para mais detalhes, consultar Nocedal e Wright (2006)
} 
esta condição não é satisfeita, ainda é possível empregá-los mediante o uso de transformações. Por exemplo, se um parâmetro $\theta$ é positivo, um método de busca linear pode ser aplicado para maximizar a $\log$-verossimilhança em função da reparametrização $\phi=\log (\theta) \in \mathbb{R}$. 


\section{Capítulo 6}

\section{Técnicas de precondicionamento para a estima- ção de modelos conjuntos}

De um modo geral, ao se resolver problemas de otimização mais complexos, é recomendável priorizar procedimentos que apresentem propriedades como robustez, precisão e estabilidade em detrimento da eficiência. Esse é um tipo de escolha razoável para muitas aplicações, uma vez que o esforço computacional necessário para a geração de resultados consistentes e acurados tende a ser diluído com o avanço de novas tecnologias (Thanedar et al., 1990; Higham, 2002). Contudo, quando se trata de análises estatísticas, a limitação da capacidade de processamento nem sempre é o único obstáculo que surge na busca por aproximações numéricas.

Na estimação de máxima verossimilhança de modelos conjuntos, uma seleção criteriosa de algoritmos com boas propriedades computacionais pode não ser suficiente para evitar dificuldades de convergência ou imprecisões nos resultados. Nesses casos, é possível que o modelo especificado não atenda as condições necessárias para ser tratado numericamente. Isso ocorre, por exemplo, quando pequenas perturbações nos parâmetros causam grandes mudanças relativas na verossimilhança em regiões próximas ao ponto de máximo. Problemas que apresentam esse tipo de comportamento local são denominados mal condicionados, e podem degradar o desempenho de métodos numéricos de otimização devido à propagação de erros ao longo das iterações (Nocedal e Wright, 2006; Gill et al., 2019).

É importante ressaltar que esse aspecto não depende dos procedimentos empregados na maximização. Isso significa que, na presença do mau condicionamento, a convergência do processo de estimação pode ser extremamente sensível à: alterações no conjunto de covariáveis; inclusões ou exclusões de efeitos aleatórios; modificações nas estruturas de covariâncias; transformações das respostas observadas; 
entre outros fatores - independente do método de otimização considerado nessa etapa. Por exemplo, se um algoritmo com ordem de convergência quadrática for usado em um problema mal condicionado, sua taxa de convergência empírica poderá ser inferior à esperada teoricamente, gerando uma perda de eficiência. Em outras situações, a aplicação de procedimentos comprovadamente estáveis no tratamento de funções mal condicionadas poderá causar problemas de instabilidade numérica e comprometer a análise estatística, fornecendo resultados inferenciais pouco precisos ou mesmo incorretos (Öztürk e Akdeniz, 2000). Esses fatos mostram que, paralelamente à busca de eficiência, o desenvolvimento de novas metodologias computacionais frequentistas deve explorar aspectos relacionados à funcionalidade dos procedimentos, bem como à correção de patologias numéricas que comprometam a performance do processo iterativo envolvido na estimação.

O objetivo deste capítulo é caracterizar os problemas de convergência recorrentes na estimação de máxima verossimilhança de modelos conjuntos e propor soluções para diminuição de seus efeitos na eficiência do processo de otimização e na precisão das aproximações geradas. As contribuições apresentadas também têm o potencial de tornar o tratamento numérico da estimação menos dependente do usuário, o que possibilita sua automatização e facilita a replicabilidade da metodologia estatística abordada.

\subsection{Condicionamento de sistemas lineares}

Em abordagens frequentistas, os algoritmos iterativos tipicamente empregados na estimação de máxima da verossimilhança envolvem, a cada passo, a resolução de sistemas de equações lineares da forma

$$
A v=w
$$

em que $\boldsymbol{v}$ e $\boldsymbol{w}$ são vetores do espaço $\mathbb{R}^{n}$ e $\boldsymbol{A}$ é uma matriz de coeficientes reais, não singular e de dimensão $n \times n$. A aplicação de métodos computacionais para solucioná-los invariavelmente produz erros de aproximação nos resultados obtidos.

A finalidade desta seção é mostrar como pequenas imprecisões introduzidas nas componentes $\boldsymbol{A}$ e $\boldsymbol{w}$ são amplificadas pelos procedimentos numéricos, afetando a qualidade da solução calculada para $\boldsymbol{v}$. Este tipo de estudo, denominado de análise de sensibilidade, é essencial para avaliar se um problema de 
otimização tem boas condições de ser tratado numericamente, permitindo identificar meios para atenuar eventuais dificuldades de convergência.

\subsubsection{Número de condição de um sistema linear}

Suponha que pequenos erros numéricos sejam introduzidos na componente $\boldsymbol{w}$. Essa perturbação, denotada por $\delta \boldsymbol{w}$, dá origem ao sistema contaminado

$$
\boldsymbol{A} \hat{\boldsymbol{v}}=\boldsymbol{w}+\delta \boldsymbol{w}
$$

cuja solução é unicamente determinada pelo vetor $\hat{\boldsymbol{v}}$. Um comportamento desejável em práticas computacionais é que o erro de aproximação da solução exata, definido por $\delta \boldsymbol{v}=\boldsymbol{v}-\hat{\boldsymbol{v}}$, seja tão pequeno quanto as imprecisões observadas em $\boldsymbol{w}$. Tal variação pode ser analisada de modo adimensional em termos dos erros relativos

$$
\varepsilon(\boldsymbol{v})=\frac{\|\delta \boldsymbol{v}\|}{\|\boldsymbol{v}\|} \quad \text { e } \quad \varepsilon(\boldsymbol{w})=\frac{\|\delta \boldsymbol{w}\|}{\|\boldsymbol{w}\|}
$$

considerando a seguinte desigualdade (Watkins, 2004, Teorema 2.2.4, p. 122)

$$
\varepsilon(\boldsymbol{v}) \leq \kappa(\boldsymbol{A}) \varepsilon(\boldsymbol{w})
$$

em que $\|\cdot\|$ representa a norma Euclidiana de um vetor do espaço $\mathbb{R}^{n}$ e $\kappa(\boldsymbol{A})$ denota uma constante positiva chamada número de condição da matriz $\boldsymbol{A}$. Quando a matriz de coeficientes é simétrica e não singular, esta medida pode ser expressa através da razão

$$
\kappa(\boldsymbol{A})=\frac{\lambda_{\max }}{\lambda_{\min }}
$$

em que $\lambda_{\max }$ e $\lambda_{\min }$ denotam o maior e o menor autovalor da matriz $\boldsymbol{A}$, respectivamente [o estudo de casos mais gerais pode ser encontrado em Watkins (2004), Quarteroni et al. (2010), entre outras referências de análise numérica].

O número de condição funciona como um fator de amplificação de erros. Valores altos de $\kappa(\boldsymbol{A})$ indicam que o erro relativo de uma solução $v$ pode aumentar drasticamente ao se realizar pequenas perturbações na componente $\boldsymbol{w}$. Nesses casos, diz-se que a matriz $\boldsymbol{A}$, ou que o sistema linear a ela associado, estão mal condicionados. Por outro lado, quando $\kappa(\boldsymbol{A})=1$, a mudança relativa na solução 
computada para $\boldsymbol{v}$ pode ser tão pequena quanto os erros introduzidos em $\boldsymbol{w}$. Esse cenário configura o caso extremo de bom condicionamento.

\subsection{Condicionamento em um problema de maximização}

Em algoritmos de otimização como os métodos de Newton e quase-Newton, descritos na Seção 5.4, a estratégia de busca linear é usada incorporando-se informações de curvatura local para encontrar um ponto de máximo. Cada iteração desses procedimentos demanda a solução de um sistema linear, cuja matriz de coeficientes é a hessiana exata ou aproximada da função objetivo. O bom condicionamento destas matrizes em regiões próximas ao ponto de máximo é essencial para se garantir a precisão das estimativas e a eficiência do processo iterativo.

Mais especificamente, quando o sistema de equações (5.12) está mal condicionado, as direções ascendentes obtidas na etapa de busca linear perdem precisão, pois são sensíveis às diversas fontes erros que são introduzidas na matriz de coeficientes e no vetor gradiente (como os erros de discretização gerados nas operações de integração e diferenciação numérica). Como consequência, um grande número de iterações é necessário até que a convergência seja atingida. Além disso, os erros carregados ao longo do processo podem comprometer o grau de acurácia das estimativas. Em casos extremos, nos quais as imprecisões são demasiadamente amplificadas, a sequência de aproximações poderá divergir, interrompendo a execução do procedimento iterativo.

O mau condicionamento da matriz hessiana de $\ell(\boldsymbol{\theta})$ em uma vizinhança de $\hat{\boldsymbol{\theta}}$ também implica na alta sensibilidade local desta função. De fato, expandindo-se a log-verossimilhança em série de Taylor de segunda ordem ao redor do ponto $\boldsymbol{\theta}_{0}$, e ao longo de uma direção $\boldsymbol{r}$, tem-se que

$$
\ell\left(\boldsymbol{\theta}_{0}+\delta \boldsymbol{r}\right)-\ell\left(\boldsymbol{\theta}_{0}\right)=\delta \boldsymbol{r}^{\top} \mathbf{U}\left(\boldsymbol{\theta}_{0}\right)+\frac{1}{2} \delta^{2} \boldsymbol{r}^{\top} \mathbf{H}\left(\boldsymbol{\theta}_{0}\right) \boldsymbol{r}+O\left(\delta^{3}\right)
$$

em que $\delta \in \mathbb{R}, \quad \mathbf{U}\left(\boldsymbol{\theta}_{0}\right)=\left(u_{1}, \ldots, u_{p}\right)^{\top}$ é o vetor escore $p$-dimensional composto por elementos que correspondem às derivadas parciais de primeira ordem

$$
u_{i}=\left.\frac{\partial \ell}{\partial \theta_{i}}\right|_{\boldsymbol{\theta}=\boldsymbol{\theta}_{0}}, \quad i=1, \ldots, p
$$

e $\mathbf{H}\left(\boldsymbol{\theta}_{0}\right)$ é a matriz hessiana de dimensão $p \times p$, cujas entradas são as derivadas parciais de segunda 
ordem

$$
h_{i j}=\left.\frac{\partial^{2} \ell}{\partial \theta_{i} \partial \theta_{j}}\right|_{\boldsymbol{\theta}=\boldsymbol{\theta}_{0}}, \quad i=1, \ldots, p, \quad j=1, \ldots, p .
$$

Como $\mathbf{U}(\hat{\boldsymbol{\theta}})=\mathbf{0}$, a expansão (6.6) em torno de $\hat{\boldsymbol{\theta}}$ é aproximada por

$$
\ell(\hat{\boldsymbol{\theta}}+\delta \boldsymbol{r})-\ell(\hat{\boldsymbol{\theta}}) \approx \frac{1}{2} \delta^{2} \boldsymbol{v}^{\top} \mathbf{H}(\hat{\boldsymbol{\theta}}) \boldsymbol{r}
$$

A simetria de $\mathbf{H}(\hat{\boldsymbol{\theta}})$ garante que os seus autovalores sejam números reais, permitindo reescrevê-la em termos da decomposição espectral

$$
\mathbf{H}(\hat{\boldsymbol{\theta}})=\boldsymbol{Q} \boldsymbol{\Lambda} \boldsymbol{Q}^{\top}
$$

em que $\boldsymbol{\Lambda}=\operatorname{diag}\left(\lambda_{1}, \ldots, \lambda_{p}\right)$ é uma matriz diagonal composta pelos autovalores de $\mathbf{H}(\hat{\boldsymbol{\theta}})$ e $\boldsymbol{Q}$ é uma matriz ortogonal, cujas colunas são formadas pelos seus autovetores associados $\boldsymbol{q}_{1}, \ldots, \boldsymbol{q}_{p}$. Quando $\lambda_{\max }$ tem grande ordem de magnitude, pequenas oscilações na direção do correspondente autovetor $\boldsymbol{q}_{\max }$ geram aumentos arbitrários na verossimilhança. Por outro lado, se $\lambda_{\min }$ é aproximadamente nulo, a vizinhança de $\hat{\boldsymbol{\theta}}$ tem a forma de um platô na direção de $\boldsymbol{q}_{\text {min }}$. Nessas regiões de baixa curvatura, há pouca variação de $\ell(\boldsymbol{\theta})$.

Esses padrões de comportamento local prejudicam o cálculo do tamanho do passo em (5.13) e fazem com que os métodos de busca linear calculem a função $\ell(\boldsymbol{\theta})$ um grande número de vezes até a conclusão dessa etapa (Gill et al., 2019; Nocedal e Wright, 2006; Nash, 2014). Note que, na estimação dos modelos conjuntos, cada avaliação exige a aproximação numérica de integrais univariadas ou múltiplas. Sendo assim, quando essa tarefa é computacionalmente intensiva a eficiência do processo pode ser consideravelmente afetada.

O mau condicionamento pode degradar a performance do processo de otimização devido (i) às falhas de convergência; (ii) à falta de precisão dos resultados; ou (iii) por apresentarem um custo computacional proibitivo, causado pelo grande número de iterações e de avaliações da função objetivo. Nesse sentido, a identificação da origem de tais problemas é o primeiro passo para desenvolver estratégias que atenuem ou eliminem os seus efeitos na maximização da verossimilhança. 


\subsection{Técnicas de precondicionamento para a estimação de modelos conjuntos}

As observações contidas em um conjunto de dados podem possuir diversas ordens de magnitude, a depender das unidades de medidas das variáveis coletadas. Em muitas aplicações, essas diferenças de escala são preservadas pela diferenciação, mantendo-se no vetor gradiente e na matriz hessiana da logverossimilhança. Os problemas de estimação que apresentam essas características tendem a ser potencialmente mau condicionados, como mostra o teorema a seguir.

Teorema 6.1 Seja A uma matriz não singular, de dimensão $n \times n$, cujas colunas são representadas por $\boldsymbol{a}_{1}, \ldots, \boldsymbol{a}_{n}$. Então, para quaisquer $i=1, \ldots, n$ e $j=1, \ldots, n$,

$$
\kappa(\boldsymbol{A}) \geq \max \left(\frac{\left\|\boldsymbol{a}_{i}\right\|}{\left\|\boldsymbol{a}_{j}\right\|}\right)
$$

Demonstração: (Watkins, 2004, Teorema 2.2.5, p. 127).

O resultado (6.9) fornece uma condição necessária para o bom condicionamento dos problemas de maximização: as colunas da matriz $\mathbf{H}(\boldsymbol{\theta})$ devem possuir, aproximadamente, a mesma ordem de magnitude em uma vizinhança de $\hat{\boldsymbol{\theta}}$.

No caso específico dos modelos conjuntos, a condição (6.9) tende a ser mais facilmente violada. A acomodação de dois submodelos distintos na mesma forma funcional (definida pela verossimilhança conjunta) faz com que as diferenças de escala entre as respostas sejam refletidas nos parâmetros do modelo, e consequentemente, na matriz hessiana da log-verossimilhança. Quanto maior a discrepância observada, maiores as chances de gerar um mau condicionamento.

Um procedimento comum na correção desses problemas é a reescala dos dados. Em modelos mais simples essa prática pode ser eficaz por padronizar os elementos de $\mathbf{H}(\boldsymbol{\theta})$, embora reduza a interpretabilidade dos parâmetros envolvidos. No entanto, quando a modelagem das respostas é feita conjuntamente, uma série de fatores podem colaborar para o mau condicionamento da maximização, como a alta dimensionalidade do espaço paramétrico ou do vetor de efeitos aleatórios. Nesses casos, a preparação dos dados pode não ser uma abordagem adequada. 
De acordo com Trefethen e Bau III (1997), a implementação eficiente de problemas desafiadores da computação científica consiste na transformação de casos aparentemente intratáveis em outros equivalentes, nos quais uma solução pode ser obtida de forma mais rápida e acurada. O precondicionamento é uma técnica que se baseia nessa ideia a fim de melhorar as características de convergência de métodos iterativos.

Precondicionar um sistema linear da forma $\boldsymbol{A v}=\boldsymbol{w}$ significa transformá-lo em um equivalente $\tilde{\boldsymbol{A}} \tilde{\boldsymbol{v}}=\tilde{\boldsymbol{w}}$, que possua um menor número de condição associado. Isso pode ser feito multiplicando-se uma matriz auxiliar $\boldsymbol{P}$, denominada matriz precondicionadora, dos seguintes modos:

i) $P A v=P w$;

ii) $\boldsymbol{A P s}=\boldsymbol{w}, \quad \boldsymbol{v}=\boldsymbol{P} s$;

iii) $\boldsymbol{P} \boldsymbol{A} \boldsymbol{P}^{\top} \boldsymbol{u}=P w, \quad v=P^{\top} u$.

A aplicação dessa técnica mapeia o espaço original $\Theta$ da função de verossimilhnça à um espaço $\tilde{\Theta}$, no qual a mesma apresenta menor sensibilidade. Nos métodos de otimização, essa é uma maneira de controlar a propagação de erros numéricos do processo iterativo, que por sua vez poderá ser executado com mais estabilidade. Entretanto, dependendo da dimensão da matriz $\boldsymbol{P}$ essa manipulação algébrica poderá exigir a multiplicação de matrizes de alta ordem, demandando um uso excessivo de memória e tempo de processamento.

Uma estratégia comum para reduzir o custo computacional dessas operações é a aplicação da reescala de parâmetros na função objetivo, conhecida como escala diagonal (Nocedal e Wright, 2006)). Para isso, a função é preparada antes de ser otimizada numericamente, mediante à reparametrização

$$
\Phi=D^{-1} \boldsymbol{\theta}
$$

em que $\boldsymbol{D}$ é uma matriz diagonal composta por fatores de escala utilizados nessa operação. Após a execução do algoritmo a solução obtida é então transformada para o seu espaço original $\Theta$. 
A mudança de variáveis (6.10) precondiciona o problema de maximização de $\ell(\boldsymbol{\theta})$ pois a cada iteração, o vetor gradiente e a matriz hessiana dessa função é substituída por

$$
\nabla \ell\left(\boldsymbol{\Phi}_{k}\right)=\boldsymbol{D}^{\top} \nabla \ell\left(\boldsymbol{\theta}_{k}\right), \quad \text { e } \quad \mathbf{H}\left(\boldsymbol{\Phi}_{k}\right)=\boldsymbol{D}^{\top} \mathbf{H}\left(\boldsymbol{\theta}_{k}\right) \boldsymbol{D}, \quad k=1,2, \ldots
$$

o que torna os sistemas lineares associados ao método menos instáveis aos erros numéricos. Existem diversas formas de se construir uma matriz diagonal $\boldsymbol{D}$ contendo fatores de escala. Quando feito de maneira sistemática o esquema descrito pode melhorar potencialmente o comportamento numérico do algoritmo. No entanto, como essa prática muda a geometria do problema original, é possível fazer a seleção de fatores a que piorem o seu condicionamento.

A próxima seção apresenta duas propostas de reparametrizações que servem para condicionar a estimação de máxima verossimilhança de modelos conjuntos. Os fatores de escala sugeridos foram baseados em particularidades do problema e em resultados algébricos, e podem ser uma solução simples e eficaz no tratamento de problemas de convergência.

\subsubsection{Reescala baseada em valores típicos das variáveis}

A operação de reescala é interpretada como uma mudança de unidade de medida das variáveis. Geralmente, essas transformações são feitas explorando-se as características específicas do problema, como a sua natureza física ou biológica. Na estimação dos modelos conjuntos, quando não se tem informações a respeito dos valores típicos de seus parâmetros, é possível extraí-las a partir do ajuste independente dos submodelos longitudinal e de sobrevivência.

Denote por $\tilde{\boldsymbol{\theta}}=\left(\tilde{\boldsymbol{\theta}}_{t}^{\top}, \tilde{\boldsymbol{\theta}}_{y}^{\top}, \tilde{\boldsymbol{\theta}}_{b}^{\top}\right)^{\top}$ o vetor contendo as estimativas dos modelos marginais. Uma proposta de fatores de escala é dada por

$$
\boldsymbol{D}_{1}=\operatorname{diag}\left(\left|\tilde{\theta}_{1}\right|, \ldots,\left|\tilde{\theta}_{p}\right|\right)
$$

Quando as ordens de magnitude dos parâmetros são preservadas pela diferenciação, essa transformação diminui o limitante superior do número de condição. Observe que, pelo Teorema (6.9)

$$
\kappa(\boldsymbol{H}(\boldsymbol{\Phi})) \geq \max \left(\frac{\left\|\boldsymbol{h}_{i}(\boldsymbol{\theta})\right\|}{\left\|\boldsymbol{h}_{j}(\boldsymbol{\theta})\right\|} \cdot \frac{\left|\tilde{\theta}_{j}\right|}{\left|\tilde{\theta}_{i}\right|}\right),
$$


para quaisquer colunas $\boldsymbol{h}_{i}(\boldsymbol{\theta})$ e $\boldsymbol{h}_{j}(\boldsymbol{\theta})$ da matriz $\mathbf{H}(\boldsymbol{\theta})$. Desse modo, as diferentes ordens de magnitude desses elementos são corrigidas pelo fator $\left|\tilde{\theta}_{j}\right| /\left|\tilde{\theta}_{i}\right|$, fazendo com que os limitantes superiores de $\kappa(\boldsymbol{H}(\boldsymbol{\Phi}))$ estejam mais próximos da unidade.

\subsubsection{Reescala baseada no precondicionador de Jacobi}

Uma técnica algébrica para se escolher um precondicionador é definir $\boldsymbol{P}=\operatorname{diag}(\boldsymbol{A})$. Se a matriz $\boldsymbol{A}$ possuir uma diagonal estritamente dominante, ou seja, para todo $i$

$$
\left|a_{i i}\right|>\sum_{j \neq i}\left|a_{i j}\right|
$$

em que $a_{i j}$ denota a entrada da $i$-ésima linha e $j$-ésima coluna da matriz $A$, então o precondicionador $\boldsymbol{P}$ pode melhorar o condicionamento de $\boldsymbol{A}$ (Watkins, 2004).

Neste trabalho, para a estimação de máxima verossimilhança dos modelos conjuntos, esse recurso é utilizado como em Gill et al. (2019). Assumindo-se que em uma vizinhança do ponto $\hat{\boldsymbol{\theta}}$ a curvatura de $\ell(\boldsymbol{\theta})$ não sofra grandes alterações, e que

$$
\mathbf{H}\left(\boldsymbol{\theta}_{k}\right) \approx \mathbf{H}(\hat{\boldsymbol{\theta}})
$$

com $\mathbf{H}\left(\boldsymbol{\theta}_{k}\right)$ diagonal dominante nesta região do espaço paramétrico, consideram-se os seguintes fatores de escala

$$
\boldsymbol{D}_{2}=\operatorname{diag}\left(\mathbf{H}\left(\boldsymbol{\theta}_{0}\right)^{-1 / 2}\right)
$$

Nestas condições, aplicando-se $\boldsymbol{D}_{2}$ em (6.10), é possível melhorar a convergência do procedimento de otimização utilizado. 


\section{Capítulo 7}

\section{Estudo de simulação}

Neste capítulo os efeitos da má especificação da componente longitudinal no contexto da modelagem conjunta são investigados por meio de um estudo de simulação, em cenários baseados no problema do ICESP. Também verifica-se o desempenho das técnicas de precondicionamento propostas no Capítulo 6 na estimação de máxima verossimilhança dos modelos conjuntos. O processo de geração dos dados é descrito na Seção 7.1. Os objetivos do estudo e os cenários analisados encontram-se especificados na Seção 7.2. Por fim, uma discussão a respeito dos resultados obtidos é apresentada na Seção 7.3.

\subsection{Geração dos dados}

O trabalho de Bender et al. (2005) foi pivotal para o desenvolvimento de técnicas eficientes para a simulação de tempos de sobrevivência provenientes de modelos de taxa de falhas proporcionais. Esta seção é dedicada à descrição desses procedimentos e de extensões discutidas em Crowther e Lambert (2013), que são empregadas na geração de dados deste capítulo.

Considere que o risco de ocorrência do evento de interesse seja explicado por meio de um modelo de taxa de falhas proporcionais, especificado como

$$
h(t \mid \boldsymbol{x})=h_{0}(t) \exp \left(\boldsymbol{x}^{\top} \boldsymbol{\gamma}\right), \quad t>0,
$$

em que $h_{0}(t)$ é a função de taxa de falha basal, $\gamma$ é um vetor contendo os parâmetros de regressão e $x$ é o vetor de variáveis explicativas, não dependentes do tempo, correspondentes. A taxa de falha acumulada é definida como

$$
H(t \mid \boldsymbol{x})=H_{0}(t) \exp \left(\boldsymbol{x}^{\top} \boldsymbol{\gamma}\right), \quad H_{0}(t)=\int_{0}^{t} h_{0}(s) d s .
$$


e as funções de sobrevivência e de distribuição acumulada relativas ao modelo (7.1) são dadas respectivamente por

$$
S(t \mid \boldsymbol{x})=\exp (-H(t \mid \boldsymbol{x})) \quad \text { e } \quad F(t \mid \boldsymbol{x})=1-\exp (-H(t \mid \boldsymbol{x})) .
$$

Note que, como a variável aleatória $\mathcal{U}=F(t \mid x)$ segue distribuição uniforme no intervalo [0,1], então a variável $\mathcal{W}=1-\mathcal{U}$ também segue distribuição uniforme no intervalo unitário (Magalhães, 2006). Assim, a realização $w$ de $\mathcal{W}$ pode ser gerada fazendo $w \sim U(0,1)$. Tendo isso em vista, denote por $t^{*}$ o tempo de sobrevivência simulado. Da equação

$$
S\left(t^{*} \mid \boldsymbol{x}\right)=u, \quad u \sim U(0,1),
$$

tem-se que, quando a taxa de falha basal é não nula para todo $t>0$, a função $H_{0}(t)$ é inversível na expressão (7.4), tornando possível isolar a variável $t^{*}$ como

$$
t^{*}=H_{0}^{-1}(v), \quad v=-\log (u) \exp \left(\boldsymbol{x}^{\top} \gamma\right)
$$

Este resultado permite obter uma relação simples entre a função de taxa falha (7.1) e os tempos de sobrevivência correspondentes. Em Bender et al. (2005), a aplicação de (7.5) à simulação de variáveis aleatórias provenientes de modelos paramétricos como o Exponencial, Weibull e Gompertz é especificada caracterizando-se as expressões para as funções $H_{0}^{-1}(t)$ associadas.

Quando se trata de cenários mais complexos, nos quais se deseja gerar dados de sobrevivência que incorporem qualquer combinação entre efeitos de interação dependentes do tempo, covariáveis dependentes do tempo, covariáveis com erros de medida e efeitos aleatórios, a função $H_{0}^{-1}(t)$ nem sempre possui uma expressão fechada, e/ou a equação (7.5) pode ser intratável analiticamente. Nesses casos, Crowther e Lambert (2013) descrevem um algoritmo para a simulação dos tempos de sobrevivência que envolve integração numérica e procedimentos iterativos para a solução de raizes de equações não lineares, esquematizado no fluxograma da Figura 7.1. A aplicação dessa metodologia na geração conjunta de dados longitudinais e de sobrevivência é especificada a seguir. 


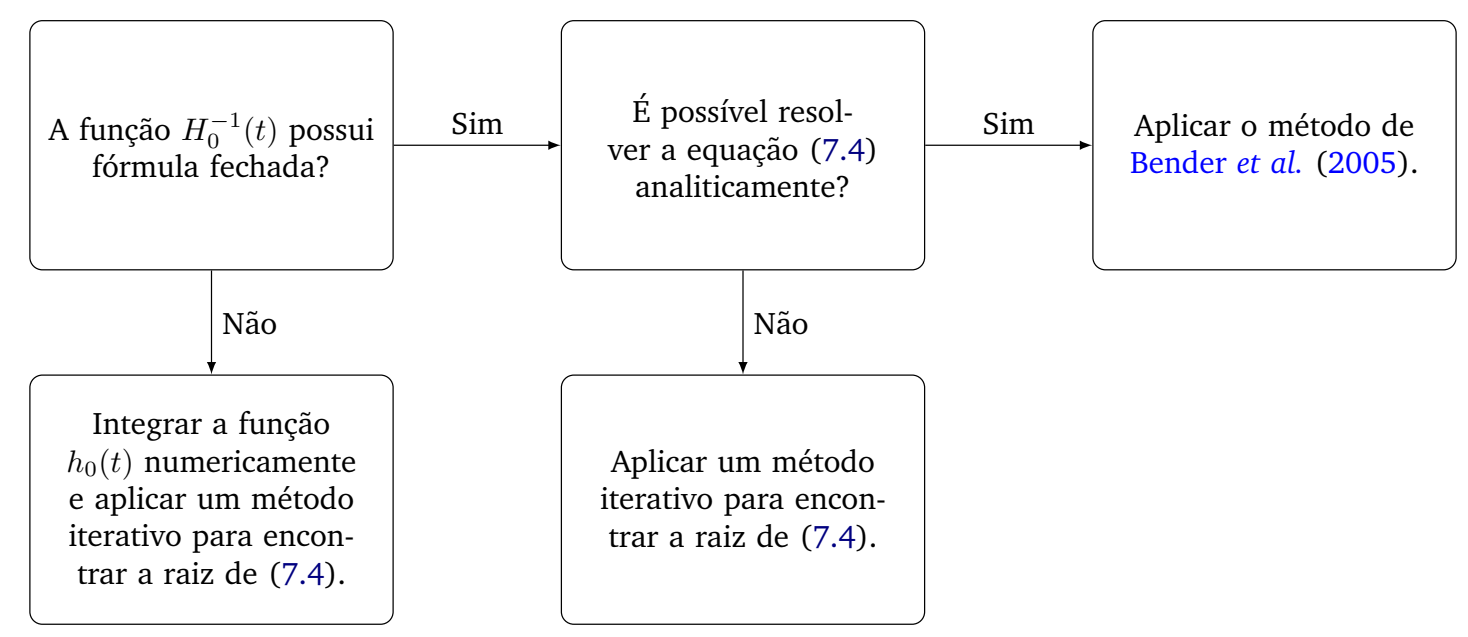

Figura 7.1: Fluxograma com as técnicas para a simulação dos tempos de sobrevivência de um modelo de riscos proporcionais abordadas em Crowther e Lambert (2013).

\subsubsection{Geração conjunta de dados longitudinais e tempos de sobrevivência}

Em geral, ao incluir a trajetória longitudinal $m_{i}(t)$ de um indivíduo $i$ no preditor linear do modelo de taxa de falha proporcionais, a taxa de falha acumulada relativa à função (4.2) passa a ser intratável analiticamente, sendo necessário recorrer à integração numérica para sua avaliação. Para o cálculo da integral em (7.2), realiza-se a seguinte mudança de variáveis

$$
H_{0}(t)=\int_{0}^{t} h_{0}(s) d s=\frac{t}{2} \int_{-1}^{1} h\left(\frac{t}{2} z+\frac{t}{2}\right) d s,
$$

de modo que o limite de integração passa a ser o intervalo $[-1,1]$. Assim, a equação (7.6) pode ser calculada através de alguma regra de quadratura, por meio da expressão

$$
\int_{0}^{t} h_{0}(s) d s \approx \sum_{i=1}^{Q} w_{i} \cdot h\left(\frac{t}{2} z+\frac{t}{2}\right)
$$

em que $w_{i}$ e $z_{i}$ são os pesos e os nós, respectivamente. Neste trabalho, considerou-se a obtenção dos pesos e nós da quadratura de Gauss-Legendre, descrito no Apêndice A.

Como consequência da aproximação (7.7), não é possível isolar o tempo simulado $t^{*}$ através de uma expressão fechada, o que exige o uso de algum procedimento iterativo. Para encontrar a raiz da equação (7.4), Crowther e Lambert (2013) sugerem a aplicação dos métodos de Brent (Brent, 2013) ou de Newton-Raphson. O método de Brent é uma combinação dos métodos da bisseção, da secante e interpolação quadrática inversa (Quarteroni et al., 2010). Comparativamente ao método de Newton- 
Raphson, apresenta como vantagem a rápida convergência sem o uso de derivadas. Entretanto, para sua aplicação na geração dos tempos de sobrevivência é importante que a equação (7.4) possua raiz definida no intervalo delimitado pelo método da bisseção. Do contrário, uma ampliação do intervalo de busca deve ser feita ou uma nova realização de $u$ deve ser gerada em (7.4).

Após a geração dos tempos de sobrevivência, as medidas longitudinais observadas são simuladas através de alguma distribuição de interesse. Neste trabalho, por exemplo, consideram-se distribuições com suporte no intervalo unitário. As respostas obtidas são então truncadas segundo os tempos de sobrevivência simulados. Desta forma, o mecanismo de abandono é incorporado no processo de geração dos dados longitudinais.

Para exemplificar o procedimento descrito suponha que as medições longitudinais sejam feitas em $t=0,15,90,180,360,540$, e que o tempo gerado para um indivíduo $i$ é $t_{i}^{*}=153$. Neste caso, as observações longitudinais são truncadas em $t=90$, descartando-se as demais. Uma representação dos dados simulados resultantes desse processo encontra-se ilustrada na Tabela 7.1.

Tabela 7.1: Ilustração das observações de uma unidade amostral de um conjunto de dados genérico, resultante do processo de simulação descrito em Crowther e Lambert (2013) para a geração de dados provenientes de um modelo conjunto.

\begin{tabular}{c|cc|cc}
\hline & \multicolumn{2}{|c|}{ Processo longitudinal } & \multicolumn{2}{c}{ Processo de sobrevivência } \\
\hline Indivíduo & Períodos de observação & Resposta longitudinal & Tempo gerado & Indicador de censura \\
\hline 1 & 0 & 0,67 & 153 & 1 \\
1 & 15 & 0,33 & 153 & 1 \\
1 & 90 & 0,21 & 153 & 1 \\
\hline
\end{tabular}

\subsection{Objetivos e cenários analisados}

Este capítulo tem como objetivos centrais (i) investigar a robustez dos modelos conjuntos para dados longitudinais limitados relativamente a uma má especificação da sua distribuição e (ii) avaliar a performance computacional dos métodos de precondicionamento propostos no Capítulo 6 na estimação de máxima verossimilhança dos modelos conjuntos. Para isso, um estudo de simulação foi realizado em cenários baseados nos principais aspectos dos dados do ICESP. 
Todas as metodologias analisadas foram implementadas no ambiente R ( $R$ Core Team, 2020). A maximização da verossimilhança foi executada com o algoritmo quase-Newton BFGS (Nocedal e Wright, 2006; Gill et al., 2019), disponibilizado no otimizador optim. A reescala dos parâmetros foi realizada antes da sua estimação. Para executá-la na função optim, basta atribuir um vetor com os fatores de escala escolhidos e inseri-lo no argumento parscale, disponível em sua lista de parâmetros de controle. A extração do código R utilizado para essa tarefa é mostrada no Apêndice C.3.

Considerou-se a geração de $R=500$ réplicas de amostras contendo $n=500$ pacientes. Para cada uma foram simuladas: (i) medições repetidas de uma resposta longitudinal univariada e restrita ao intervalo $(0,1]$, tomadas nos instantes $t=0,15,90,180,360$, e 540; (ii) variáveis explicativas não dependentes do tempo e (iii) tempos de sobrevivência censurados à direita, considerando a censura do Tipo I através da especificação de um tempo máximo de acompanhamento de $\tau=720$ dias. Os percentuais de censura à direita usados neste estudo foram $P_{c}=0 \%$ e $20 \%$.

Para simular cenários com respostas longitudinais contínuas no intervalo $(0,1)$, contendo uma determinada proporção de observações unitárias, os dados longitudinais foram gerados a partir dos modelos de regressão beta e beta inflacionado em um, especificados com um intercepto aleatório. No modelo beta, as proporções de observações unitárias foram geradas separadamente, e utilizadas para contaminar os dados distribuídos no intervalo $(0,1)$ de modo aleatório; ao passo que no modelo inflacionado, foram determinadas através do parâmetro de mistura $\xi$. Essa é uma forma de se representar as observações ocasionais de escores máximos de qualidade de vida dos dados do ICESP.

Para os dois modelos longitudinais considerados na geração dos dados, os valores dos interceptos aleatórios foram simulados por meio da distribuição normal como $b_{0 i} \sim N\left(0, \sigma_{b_{0}}^{2}\right)$, e os preditores lineares (4.4) e (4.8) foram definidos, respectivamente, pela expressão

$$
\eta_{i}(t)=\beta_{0}+\beta_{1} t+\beta_{2} x_{i}+b_{0 i}
$$

em que $\eta_{i}(t)=\operatorname{logito}\left(m_{i}(t)\right)$ para o modelo de regressão beta e $\eta_{i}(t)=\operatorname{logito}\left(\mu_{i}(t)\right)$ para o modelo beta inflacionado, $t$ denota a variável tempo e $x$ a variável quimioterapia prévia - não, descrita na Tabela B.1. Os percentuais de observações unitárias nos dados longitudinais gerados foram $P_{\max }=0,5 \%, 10 \%$ e $20 \%$. 
Tabela 7.2: Valores dos parâmetros utilizados na geração conjunta dados longitudinais e de sobrevivência.

\begin{tabular}{|c|c|c|c|c|}
\hline \multirow[b]{2}{*}{ Processos } & \multicolumn{2}{|c|}{ Modelo BEI-WEI } & \multicolumn{2}{|c|}{ Modelo BE-WEI } \\
\hline & Parâmetros & Valor & Parâmetros & Valor \\
\hline \multirow{5}{*}{ Longitudinal } & $\beta_{0}$ & -0.500 & $\beta_{0}$ & $-0,052$ \\
\hline & $\beta_{1}$ & 0,001 & $\beta_{1}$ & 0,001 \\
\hline & $\beta_{2}$ & 0,010 & $\beta_{2}$ & 0,010 \\
\hline & $\sigma_{b 0}$ & 0,200 & $\sigma_{b 0}$ & 0,200 \\
\hline & $\phi$ & 0,600 & $\phi$ & 1,140 \\
\hline \multirow{3}{*}{ Sobrevivência } & $\gamma_{0}$ & $-1,000$ & $\gamma_{0}$ & $-3,130$ \\
\hline & $\gamma_{1}$ & $-0,900$ & $\gamma_{1}$ & $-0,900$ \\
\hline & $\rho$ & 0,630 & $\rho$ & 0,580 \\
\hline
\end{tabular}

Para a geração dos tempos de sobrevivência empregou-se o modelo de taxa de falha proporcionais Weibull, caracterizado pela taxa de falha

$$
h_{i}\left(t \mid \mathcal{M}_{i}(t), \boldsymbol{\omega}_{i}\right)=\rho t^{\rho-1} \cdot \exp \left\{\gamma_{0}+\gamma_{1} \omega_{i}+\alpha m_{i}(t)\right\}
$$

em que $\omega$ representa a variável tipo de admissão na UTI-não planejada.

Um dos aspectos investigados referente ao objetivo (I) é a capacidade dos modelos conjuntos propostos em captar a associação dos processos longitudinal e de sobrevivência, mediante a ocorrência de observações longitudinais circunstanciais de pontos extremos. Uma vez que o parâmetro que mede esse efeito pode assumir valores reais, procurou-se simular situações em que houvesse uma associação mais baixa e outra em que ela fosse maior. Deste modo, as seguintes atribuições foram consideradas $\alpha=-1$ e $\alpha=-4$. Os valores dos outros parâmetros empregados na geração dos dados encontram-se dispostos na Tabela 7.2.

Após a geração das respostas de interesse, foram realizados os ajustes dos modelos conjuntos cuja especificação da componente longitudinal foi feita através dos modelos de regressão beta, beta inflacionada em um e normal com intercepto aleatório, e a especificação da componente de sobrevivência foi feita por meio do modelo de taxa de falha proporcionais Weibull. Neste trabaho, para a aplicação do modelo beta, as observações unitárias foram mapeadas no intervalo $(0,1)$ através da transformação (2.2). No caso do modelo normal, considerou-se a modelagem da transformação logito (2.4) das respostas longitudinais simuladas. Para facilitar a leitura deste capítulo e dos próximos, abreviam-se os modelos descritos respectivamente como BE-WEI, BEI-WEI e LN-WEI. 


\subsubsection{Métricas de desempenho}

A robustez dos modelos propostos mediante a má especificação da distribuição das respostas longitudinais foi investigada avaliando-se o processo de estimação em termos do viés $(V I)$, viés relativo $(V I R)$, raiz do erro quadrático médio $(R E Q M)$ e raiz do erro quadrático médio relativo ( $R E Q M R)$, definidos respectivamente por

$$
\begin{gathered}
V I(\hat{\boldsymbol{\theta}})=\hat{\boldsymbol{\theta}}-\boldsymbol{\theta}, \quad V I R(\hat{\boldsymbol{\theta}})=\frac{\overline{\hat{\boldsymbol{\theta}}}-\boldsymbol{\theta}}{\boldsymbol{\theta}}, \quad \overline{\hat{\boldsymbol{\theta}}}=\frac{1}{R} \sum_{l=1}^{R} \hat{\boldsymbol{\theta}}_{l}, \\
R E Q M(\hat{\boldsymbol{\theta}})=\sqrt{\frac{1}{R} \sum_{l=1}^{R}(\hat{\boldsymbol{\theta}}-\boldsymbol{\theta})^{2},} \quad R E Q M R(\hat{\boldsymbol{\theta}})=\sqrt{\frac{1}{R} \sum_{l=1}^{R}\left(\frac{\hat{\boldsymbol{\theta}}-\boldsymbol{\theta}}{\boldsymbol{\theta}}\right)^{2}} .
\end{gathered}
$$

Além disso, com o objetivo de verificar a capacidade desses modelos de captar diferentes graus de dependência entre os processos longitudinal e de sobrevivência, para cada um dos cenários investigados foi considerado o percentual de parâmetros de associação significativos estimados nas 500 réplicas geradas, de acordo com o p-valor do teste de Wald.

A comparação da performance computacional das metodologias desenvolvidas para o condicionamento da maximização baseou-se em duas medidas de eficiência, ambas relacionadas ao número de avaliações fundamentais da função otimizada, termo alusivo à qualquer subrotina chamada pelo algoritmo durante o processo de maximização (Beiranvand et al., 2017). Essa métrica foi escolhida pois não depende das características do processador, da plataforma utilizada ou de aspectos referentes à implementação, podendo ser empregada em diversas situações gerais.

Por exemplo, um procedimento iterativo pode ser ineficiente por precisar de um grande número de iterações até a convergência quando comparado à outros. Contudo, é possível que a paralelização de suas rotinas ou que sua implementação em outras linguagens de programação reduzam significativamente o tempo de execução. Nessas circunstâncias, se o tempo de processamento for empregado como métrica de desempenho, será mais difícil discriminar a performance dos processos iterativos, visto que a eficiência do processo pode estar ligada a fatores não relacionados às metodologias numéricas analisadas. 
No caso dos modelos conjuntos, grande parte do esforço computacional em análises frequentistas é atribuída à integração numérica da verossimilhança. Quanto menor o número de chamadas dessa função em um processo convergente, maior a sua eficiência. Sendo assim, a primeira medida aplicada neste trabalho é o número de avaliações da verossimilhança, denotada por $\mathcal{N}_{1}$. A segunda é uma estimativa para essa quantidade, em situações nas quais o vetor gradiente é calculado por diferenças finitas. Tal métrica é definida como

$$
\mathcal{N}_{2}=\mathcal{N}_{1}+p \cdot \mathcal{N}_{\text {iter }},
$$

em que $p$ é o número de parâmetros do modelo e $\mathcal{N}_{\text {iter }}$ é o número de iterações realizadas.

\subsection{Resultados}

\subsubsection{Desempenho dos estimadores de máxima verossimilhança}

As Tabelas 7.3, 7.4, 7.5 e 7.6, apresentam os verdadeiros valores dos parâmetros utilizados na geração dos dados, a média das estimativas dos parâmetros, o viés $(V I)$, viés relativo $(V I R)$, raiz do erro quadrático médio $(R E Q M)$ e raiz do erro quadrático médio relativo $(R E Q M R)$ para as estimativas dos parâmetros dos modelos BEI-WEI, BE-WEI e LN-WEI. Foram geradas amostras a partir dos modelos BEI-WEI e BE-WEI, com diferentes percentuais de censura à direita $\left(P_{C}=0 \%, 20 \%\right)$ e de observações iguais a um $\left(P_{\max }=0 \%, 10 \%\right.$ e $\left.20 \%\right)$. Neste estudo, os parâmetros dos modelos de regressão beta e beta inflacionado são comparáveis, pois descrevem as trajetórias de escores de qualidade de vida que pertencem ao intervalo $(0,1)$. No caso do modelo normal, as análises serão voltadas para os parâmetros envolvidos no submodelo de sobrevivência correspondente.

Considerando as amostras simuladas a partir do modelo BEI-WEI com tempos de sobrevivência não censurados, observou-se que quando pequenas proporções de medições longitudinais unitárias estavam contidas na amostra $\left(P_{\max }=0,5 \%\right)$, os modelos conjuntos BEI-WEI e BE-WEI apresentaram performances semelhantes, no sentido de que não ocorreram diferenças importantes de suas estimativas em termos de viés e erro quadrático médio. Nessas situações, a transformação das respostas longitudinais unitárias para a aplicação do modelo de regressão beta não comprometeu expressivamente a qualidade das estimativas. Por outro lado, quando maiores percentuais de uns $\left(P_{\max }=10 \%\right.$ e $\left.20 \%\right)$ foram gerados nas amostras, na maior parte dos parâmetros analisados houve uma piora no viés e no erro 
quadrático médio associados às estimativas dos parâmetros do modelo BE-WEI, o que é um reflexo da má especificação da sua componente longitudinal.

Com relação à presença de censura à direita, a interpretação dos resultados obtidos deve levar em conta a análise simultânea das respostas, baseada na magnitude de sua associação. Quando a dependência induzida nos processos simulados foi mais fraca, caso em que especificou-se um valor de associação $\alpha=-1$, verificou-se que, embora as observações censuradas tenham prejudicado a estimação no processo de sobrevivência através do aumento do viés ou do erro quadrático médio das estimativas, poucas diferenças foram notadas para os parâmetros do modelo longitudinal, comparativamente aos ajustes às amostras sem a ocorrência de censura. Em contrapartida, nos cenários em que $\alpha=-4$, os efeitos da censura no viés e no erro quadrático médio das estimativas foram relevantes para maioria dos parâmetros, inclusive nos relacionados à componente longitudinal. Esse comportamento ocorre pois, quanto mais próxima a associação é de zero, os processos descritos pelos submodelos tendem a possuir um maior grau de independência.

Para todas as configurações de dados avaliadas, as estimativas dos parâmetros de sobrevivência obtidas através do modelo conjunto LN-WEI apresentaram um desempenho inferior ao dos modelos conjuntos propostos para respostas longitudinais limitadas, mostrando a importância da modelagem adequada da componente longitudinal nos cenários estudados.

Além de dificultar a determinação de importantes fatores que explicam taxa de ocorrência para o evento de interesse, a má especificação das medidas repetidas pode comprometer a detecção da dependência entre os processos longitudinal e de sobrevivência. Nas Tabelas 7.7 e 7.8 estão dispostos os percentuais de associações significativas que foram estimados nas 500 réplicas geradas, utilizando diferentes proporções de respostas longitudinais unitárias nas amostras e tempos de sobrevivência não censurados. Quando os dados longitudinais foram simulados do modelo inflacionado e a dependência entre as respostas foi mais fraca $(\alpha=-1)$, o modelo BEI-WEI detectou um maior número de associações significativas do que os outros. Considerando-se amostras geradas com dados longitudinais beta contaminados com uns e dependência mais fraca, também percebe-se um melhor desempenho dos modelos conjuntos BEI-WEI para essa finalidade. 
Nos casos em que a associação induzida entre os processos foi mais forte $(\alpha=-4)$, os três modelos ajustados forneceram associações significativas para todos os dados gerados nas 500 réplicas. Ressalta-se que, mesmo diante da má especificação da componente longitudinal, o modelo inflacionado mostrou maior estabilidade nos diferentes cenários para captar a dependência das respostas, mostrando-se mais robusto às observações unitárias contidas nas amostras geradas.

\subsubsection{Performance computacional}

Relativamente à performance computacional, para ilustrar o funcionamento das técnicas propostas neste trabalho, analisa-se o desempenho da estimação de máxima verossimilhança dos modelos conjuntos BEI-WEI e BE-WEI em amostras geradas pelo modelo BEI-WEI, para as quais o percentual de censura é $P_{c}=0 \%$, o parâmetro de associação é $\alpha=-4$ e os percentuais de observações unitárias são $P_{\max }=0,5 \%, 10 \%$ e $20 \%$.

Nas Figuras 7.2 e 7.3 são apresentados os números de condição da matriz $H(\hat{\boldsymbol{\theta}})$ em uma escala logarítmica, e as métricas $\mathcal{N}_{1}$ e $\mathcal{N}_{2}$ para o ajuste dos modelos BEI-WEI e BE-WEI, respectivamente. Foram consideradas a otimização da verossimilhança sem o precondicionamento e com os fatores de escala discutidos no Capítulo 6. De um modo geral, observou-se que:

i) Com relação ao ajuste do modelo BEI-WEI, verificou-se que quando o percentual de observações unitárias foi muito baixo $\left(P_{\max }=0,5 \%\right)$ houve uma maior variabilidade entre os números de condição obtidos através do precondicionador de Jacobi. Como consequência, maiores variabilidades são observadas nos números de avaliações $\mathcal{N}_{1}$ e $\mathcal{N}_{2}$. Esse comportamento é justificado pelo fato desse fator de escala se basear na matriz $H(\hat{\boldsymbol{\theta}})$, refletindo a falta de precisão das estimativas. Por outro lado, quando a proporção de uns aumenta, mais informações são inseridas para se estimar a o parâmetro $\xi$, resultando maior precisão a $\hat{\boldsymbol{\theta}}$. Por essa razão, menores variabilidades são notadas para as medidas referentes ao precondicionador de Jacobi quando $P_{\max }=10 \%$ e $20 \%$. O fator de escala baseado nos valores típicos dos parâmetros gerou métricas com pouca variabilidade para todos os percentuais de uns considerados, sendo o mais indicado para os cenários investigados; 


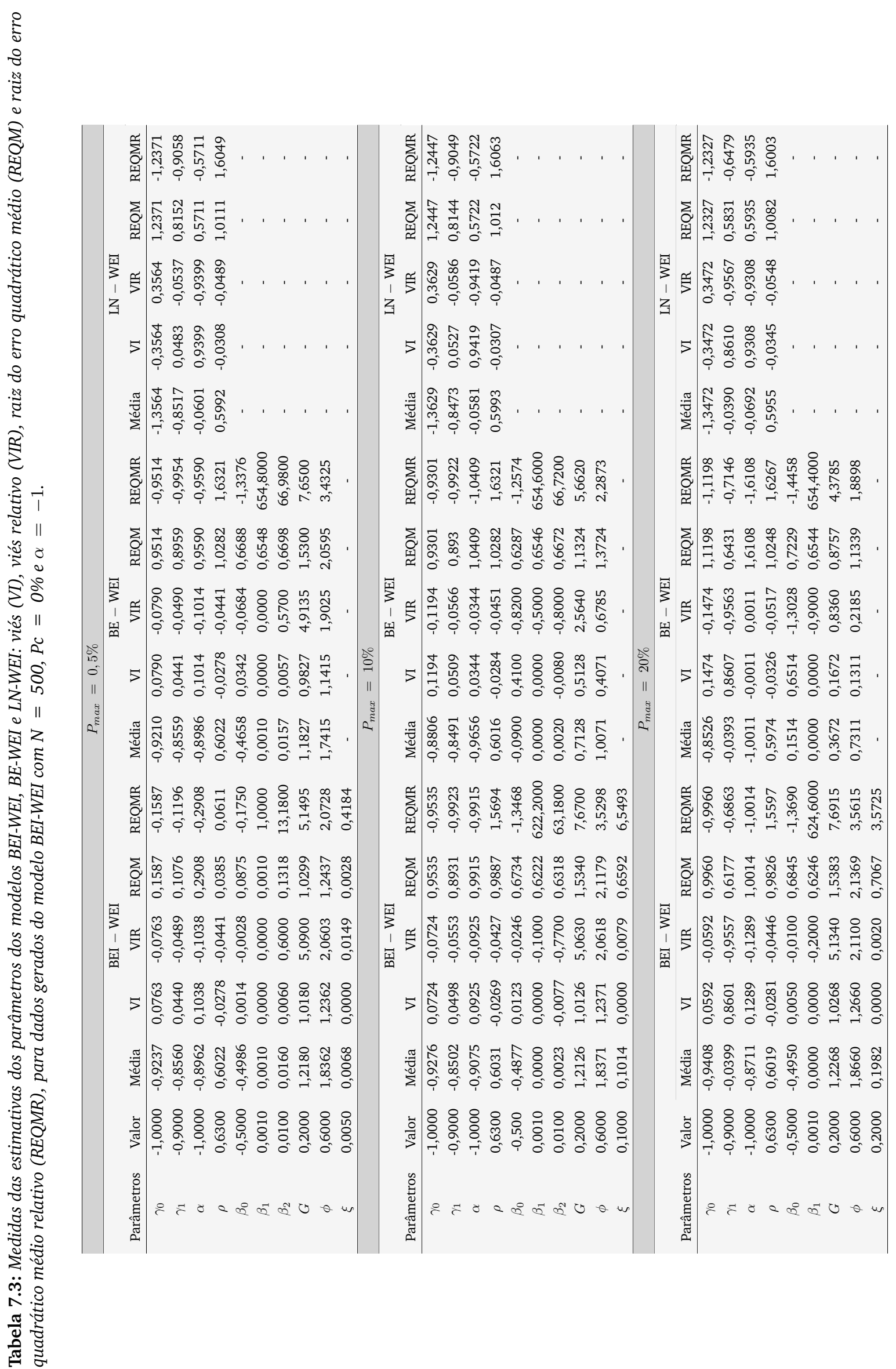




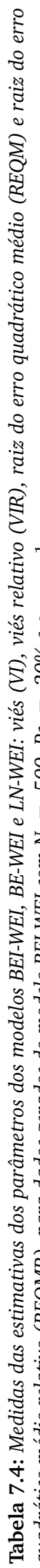

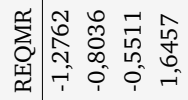

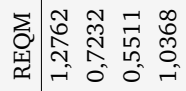

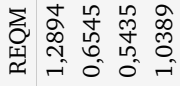

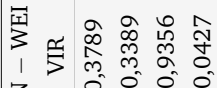

z

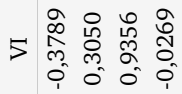

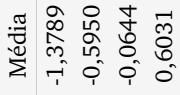

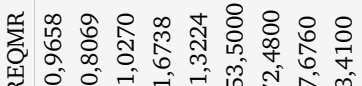

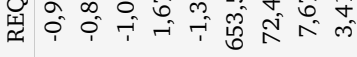

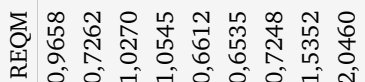

車

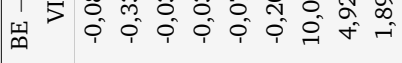

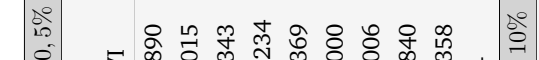

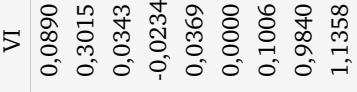

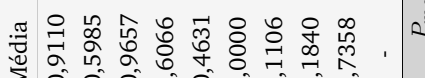

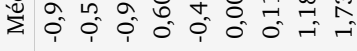

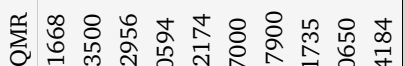

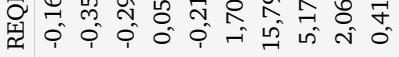

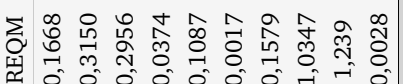

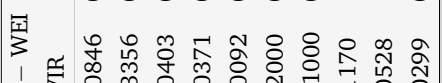

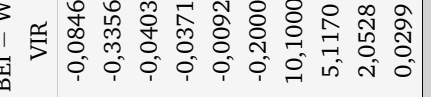

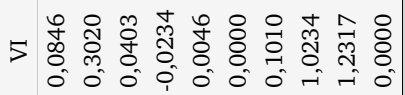

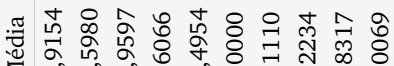

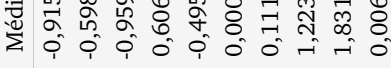

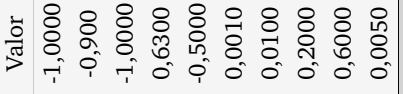

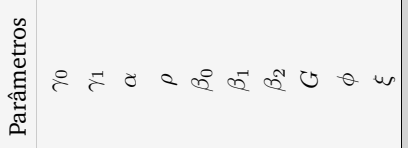

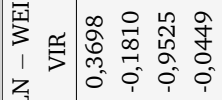

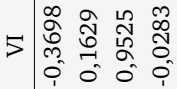

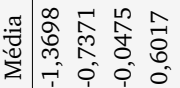

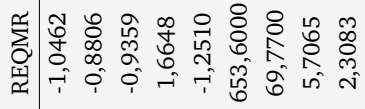

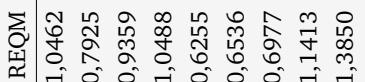

零

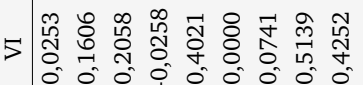

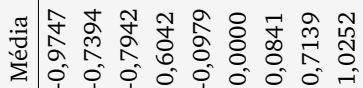

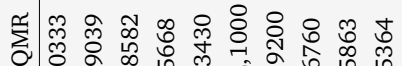

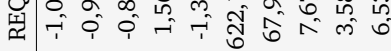

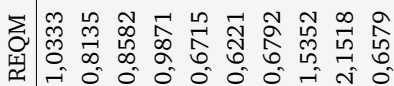

牙

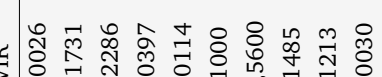

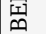

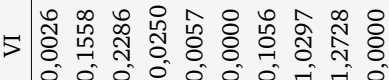

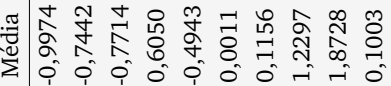

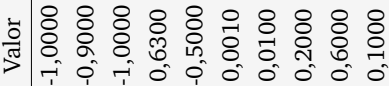

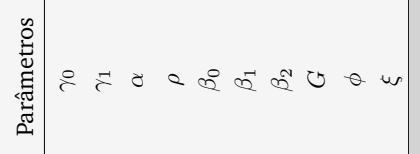

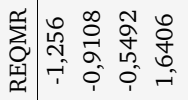

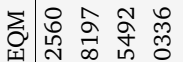

뵈석

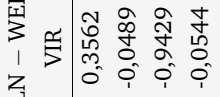

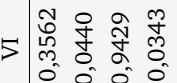

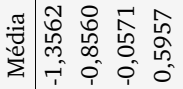

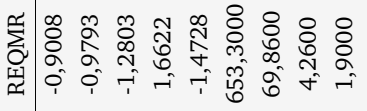

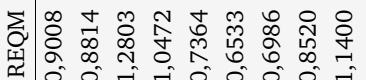

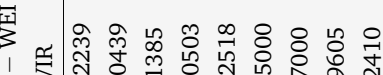

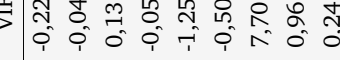

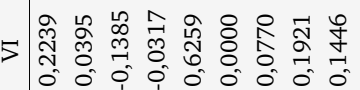

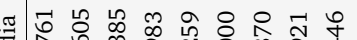

幽定

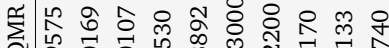

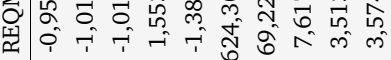

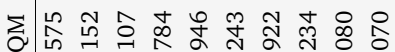

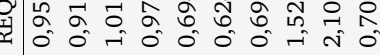

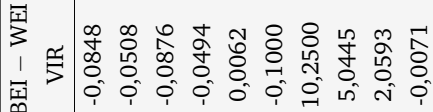

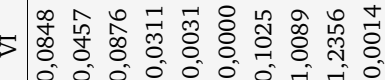

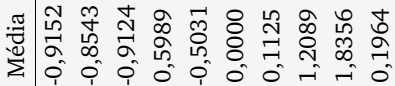

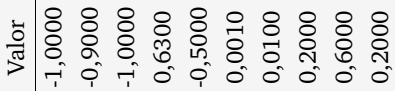

善 


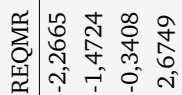

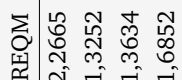

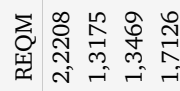

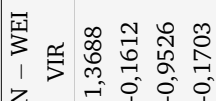

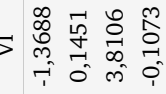

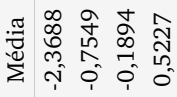

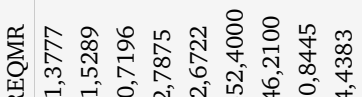

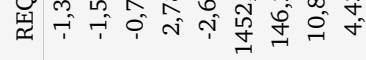

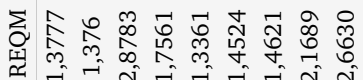

㫄

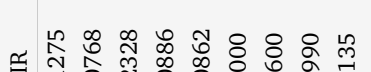

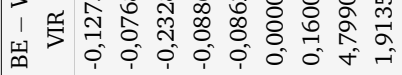

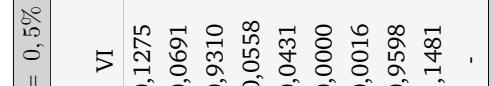

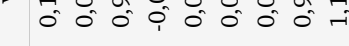

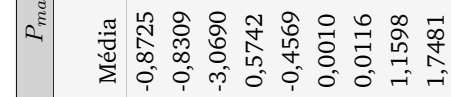

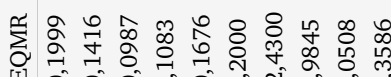

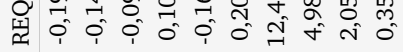

引

可

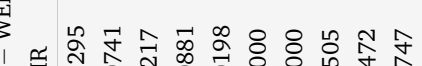

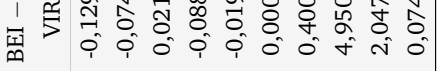

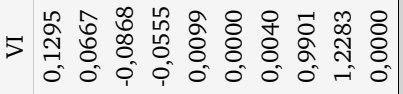

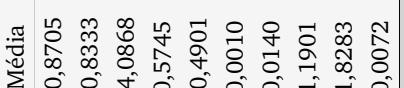

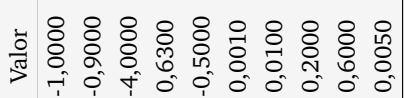

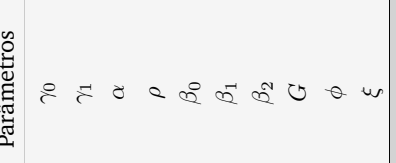

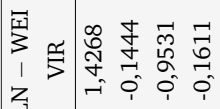

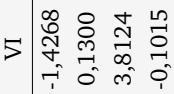

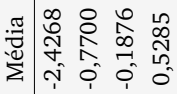

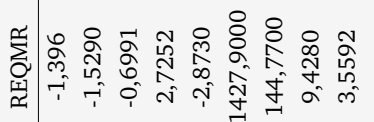

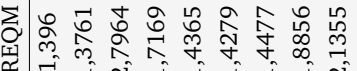

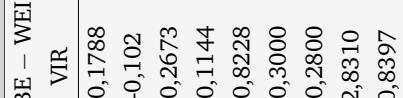

11

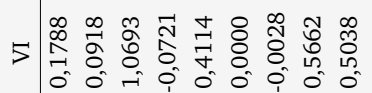

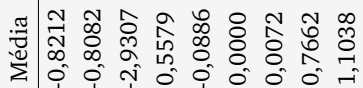

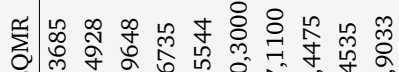

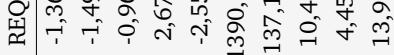

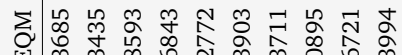

贯

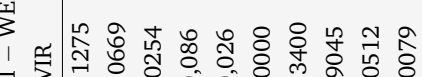

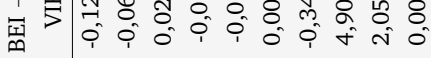

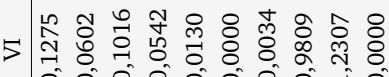

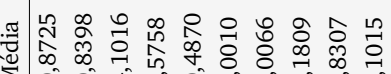

एँ.

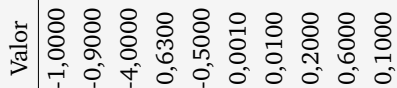

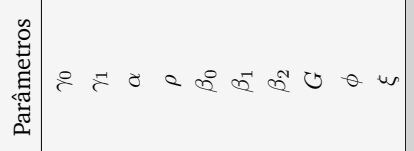

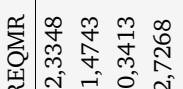

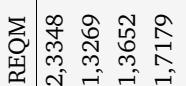

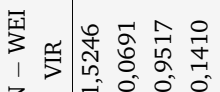

z

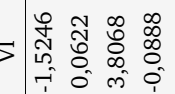

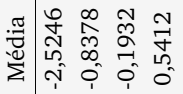

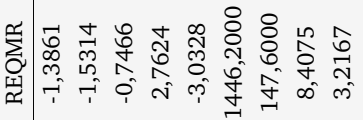

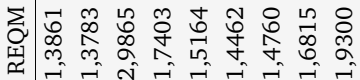

雨

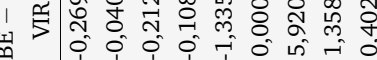

5 突。

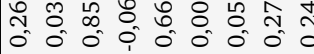

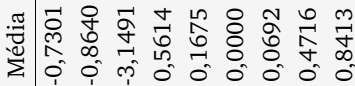

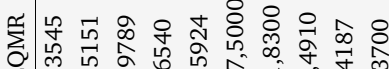

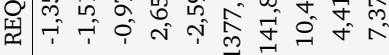

¿

牙崖

잉 \&

舟

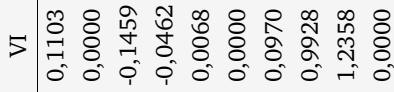

孚

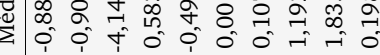

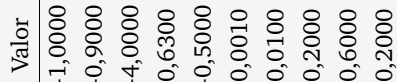

䓪 


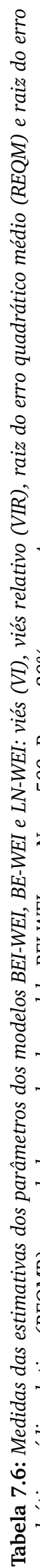

进总

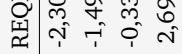

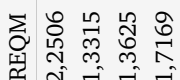

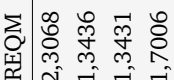

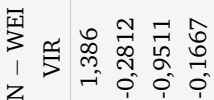

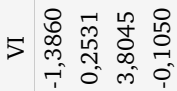

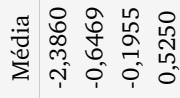

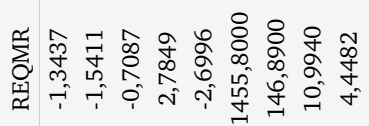

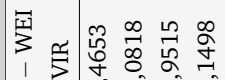

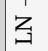

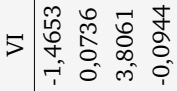

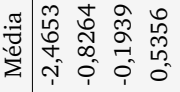

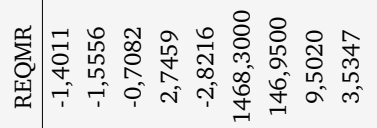

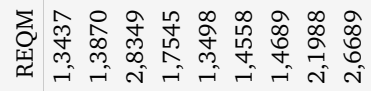

䪵

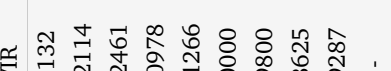

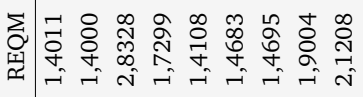

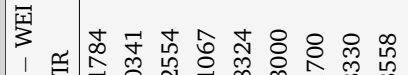

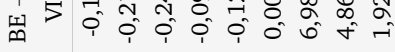

(1)

䁬 5 î

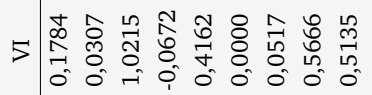

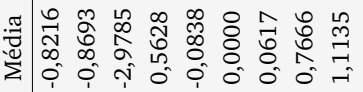

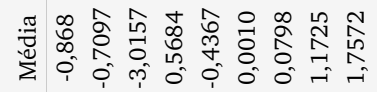

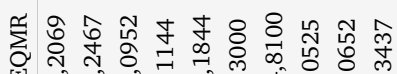

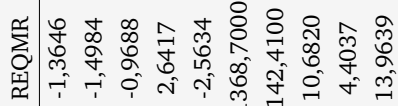

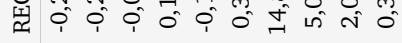

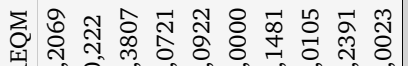

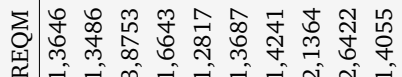

要

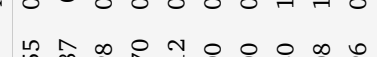

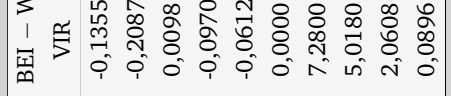

藏

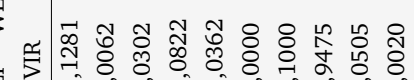
5 员

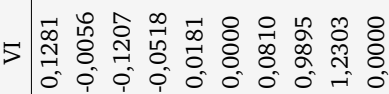

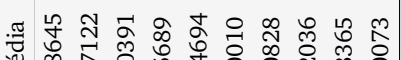

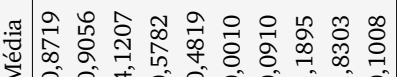

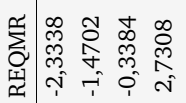

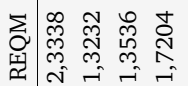

雷

z

5 >

वे 0

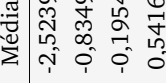

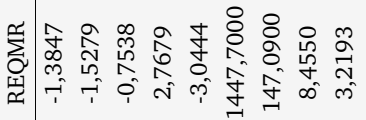

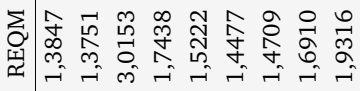

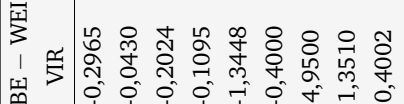

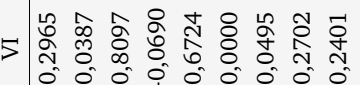

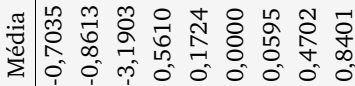

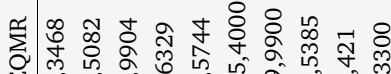

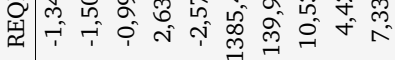

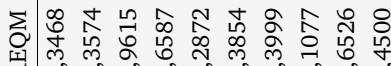

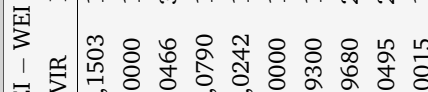

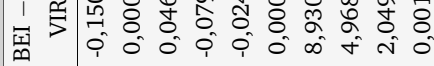

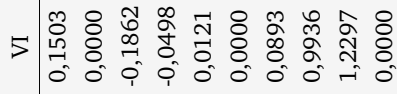

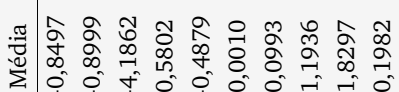

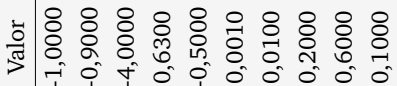

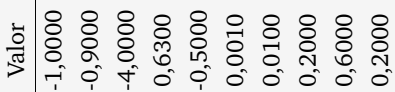

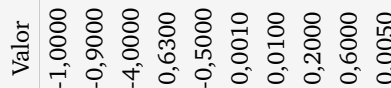

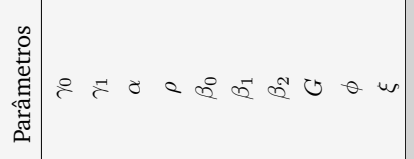

莺

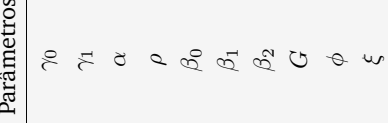


ii) No que diz respeito ao ajuste do modelo BE-WEI, nota-se que o aumento das observações de uns nas amostras gerou uma pequena amplificação nos números de condição obtidos. O fator de escala de Jacobi gerou números de avaliação mais dispersos com o aumento das observações unitárias nos dados. Esse comportamento foi possivelmente ocasionado pela imprecisão que é causada nas estimativas ao se transformar os pontos de fronteira para o ajuste.

Destaca-se que em todos os cenários analisados, os fatores de escala propostos reduziram significativamente o número de condição. Este resultado mostra o potencial da metodologia em melhorar as características de convergência de um método quase-Newton, garantindo maior estabilidade do processo iterativo e a robustez dos resultados diante de diferentes tipos de erros numéricos. Em particular, em cenários mais desafiadores para a modelagem conjunta, que exigem, por exemplo, a incorporação de múltiplos efeitos aleatórios para a descrição das médias individuais, a redução das avaliações da verossimilhança será refletida no tempo de processamento, agregando eficiência ao processo de estimação. 
Tabela 7.7: Percentuais de estimativas de associações significativas obtidas através do ajuste dos modelos BEI-WEI, BE-WEI e LN-WEI nas 500 réplicas geradas a partir do modelo BEI-WEI, considerando diferentes graus de dependência $(\alpha)$ entres as respostas simuladas e percentuais de observações unitárias na amostra $\left(P_{\max }\right)$ iguais a $0,5 \%, 10 \%$ e $20 \%$.

\begin{tabular}{cccc}
\hline \multicolumn{4}{c}{$\alpha=-1$} \\
\hline Modelos ajustados & \multicolumn{3}{c}{$P_{\max }(\%)$} \\
\hline & 0,5 & 10 & 20 \\
BEI - WEI & 93 & 83 & 63 \\
BE - WEI & 92 & 72 & 31 \\
LN - WEI & 83 & 62 & 22 \\
\hline \multicolumn{4}{c}{} \\
\hline Modelos ajustados & $\alpha=-4$ & $P_{\max }(\%)$ \\
\hline & 0,5 & 10 & 20 \\
BEI - WEI & 100 & 100 & 100 \\
BE - WEI & 100 & 100 & 98 \\
LN - WEI & 100 & 100 & 99 \\
\hline
\end{tabular}

Tabela 7.8: Percentuais de estimativas de associações significativas obtidas através do ajuste dos modelos BEI-WEI, BE-WEI e LN-WEI nas 500 réplicas geradas a partir do modelo BE-WEI, considerando diferentes graus de dependência $(\alpha)$ entres as respostas simuladas e percentuais de observações unitárias na amostra $\left(P_{\max }\right)$ iguais a $0,5 \%, 10 \%$ e $20 \%$.

\begin{tabular}{cccc}
\hline \multicolumn{4}{c}{$\alpha=-1$} \\
\hline Modelos ajustados & \multicolumn{3}{c}{$P_{\max }(\%)$} \\
\hline & 0,5 & 10 & 20 \\
BEI - WEI & 73 & 71 & 44 \\
BE - WEI & 61 & 59 & 34 \\
LN - WEI & 49 & 48 & 22 \\
\hline \multicolumn{4}{c}{$P_{\max }(\%)$} \\
\hline Modelos ajustados & $\alpha=-4$ \\
\hline & 0,5 & 10 & 20 \\
BEI - WEI & 100 & 100 & 100 \\
BE - WEI & 100 & 100 & 100 \\
LN - WEI & 100 & 99 & 100 \\
\hline
\end{tabular}



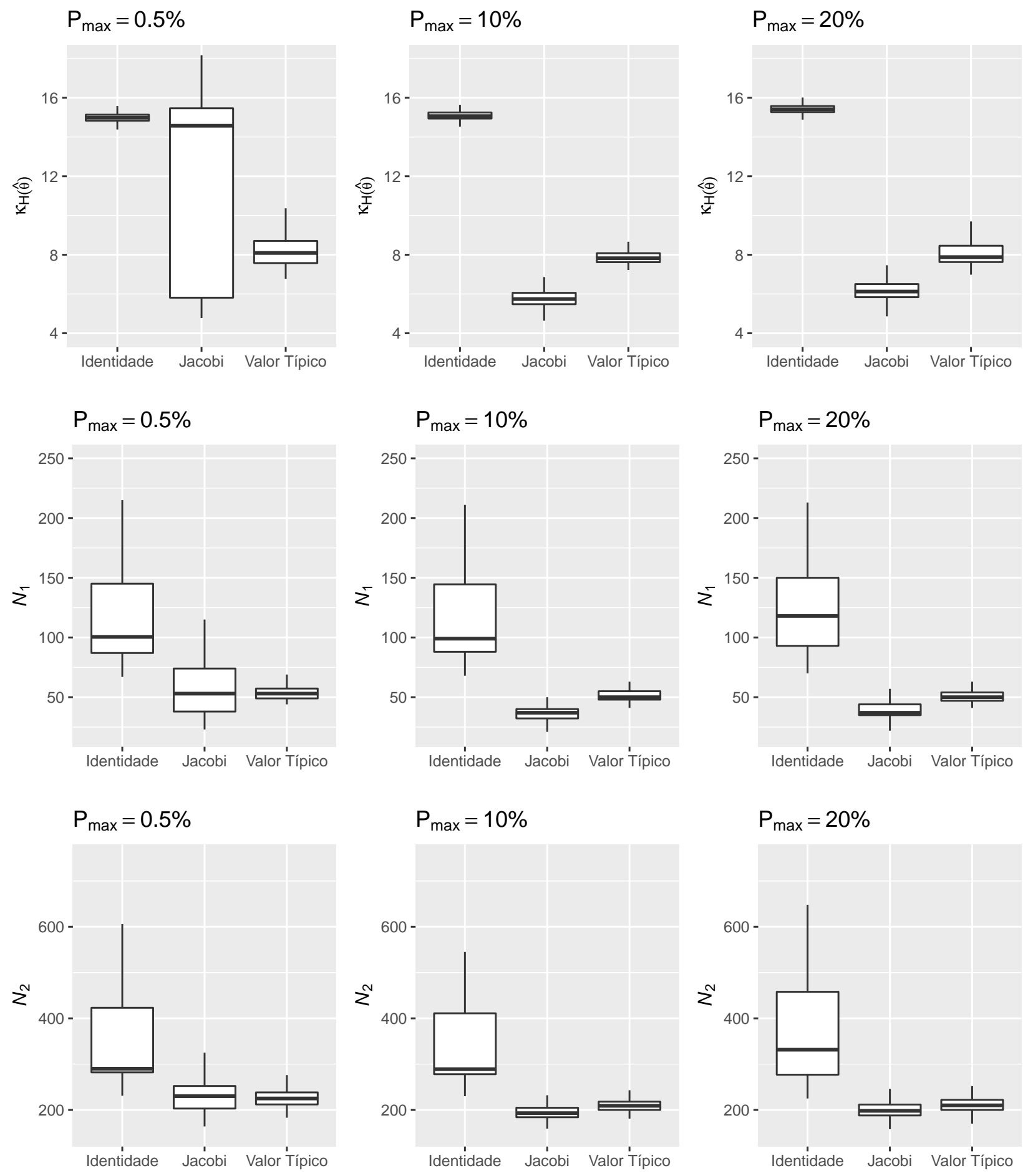

Figura 7.2: Boxplots com as medidas de desempenho computacional da estimação de máxima verossimilhança do modelo BEI-WEI considerando o seu ajuste às 500 réplicas dos dados gerados pelo modelo BEI-WEI e utilizando as técnicas de precondicionamento propostas, 

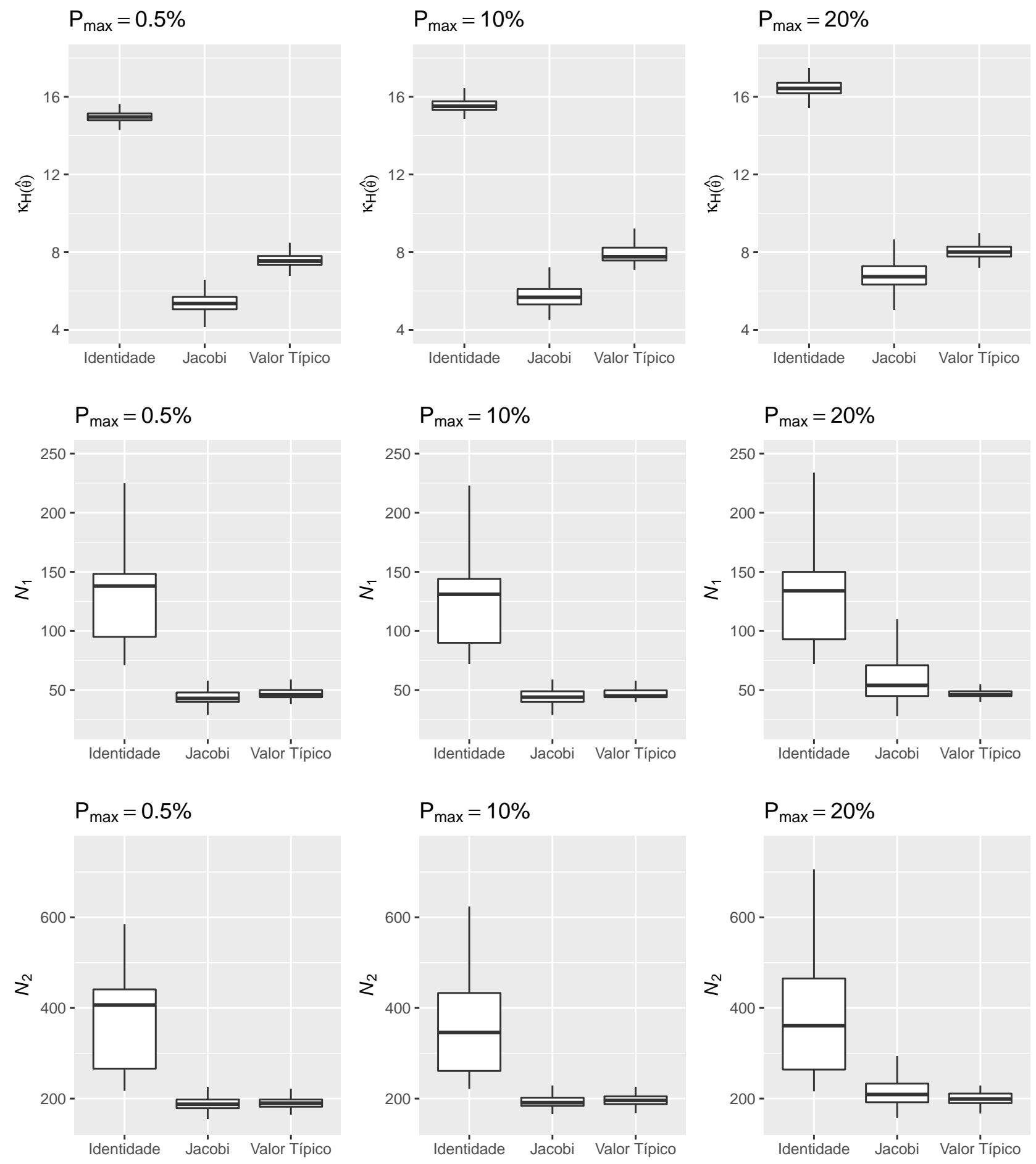

Figura 7.3: Boxplots com as medidas de desempenho computacional da estimação de máxima verossimilhança do modelo BE-WEI considerando o seu ajuste às 500 réplicas dos dados gerados pelo modelo BEI-WEI e utilizando as técnicas de precondicionamento propostas, 


\section{Capítulo 8}

\section{Análise dos dados do ICESP}

Neste capítulo, a abordagem conjunta proposta é aplicada ao conjunto de dados dos 803 pacientes oncológicos do ICESP, introduzidos no Capítulo 2. Para reduzir os problemas de condicionamento e melhorar as características de convergência da estimação de máxima verossimilhança, o uso dos fatores de escala do Capítulo 6 foi explorado na Seção 8.2. A investigação da associação entre os escores de qualidade de vida, obtidos através do instrumento EQ-5D, e do processo de sobrevivência, é apresentada na Seção 8.1. Já a análise da performance dos modelos conjuntos na predição das probabilidades de sobrevivência é feita na Seção 8.4

\subsection{Especificação dos modelos conjuntos}

O objetivo principal do estudo do ICESP foi explicar a sobrevida, a qualidade de vida e os anos de vida ajustados pela qualidade de vida de pacientes admitidos em unidades de terapia intensiva, em função de informaçãoes recolhidas no início do acompanhamento. Sob o respaldo dos resultados fornecidos em Normilio-Silva et al. (2016), um conjunto de covariáveis que apresentaram forte correlação com as respostas de interesse foi selecionado para as análise deste capítulo, e encontram-se resumidos na Tabela B.1. As variáveis mais significativas para a descrição das respostas longitudinais e de sobrevivência foram identificadas para cada submodelo separadamente, como sugerido em Wu et al. (2012) e em Soto et al. (2020). Dessa forma, a componente longitudinal foi explicada por meio dos modelos de regressão beta e beta inflacionado com intercepto aleatório, especificando-se os seus preditores lineares como

$$
\begin{aligned}
\eta_{i}(t) & =\beta_{0}+\beta_{1} t+\beta_{2} E \operatorname{cog}-1_{i}+\beta_{3} E \operatorname{cog}-2_{i}+\beta_{4} E \operatorname{cog}-3_{i} \\
& +\beta_{5} E \operatorname{cog}-4_{i}+\beta_{6} \text { Radio }_{i}+b_{0 i},
\end{aligned}
$$


em que $\eta_{i}(t)=\operatorname{logito}\left(m_{i}(t)\right)$ para o modelo (4.4), $\eta_{i}(t)=\operatorname{logito}\left(\mu_{i}(t)\right)$ para o modelo (4.8) e Ecog-1, Ecog-2, Ecog-3, Ecog-4 e Radio são variáveis binárias auxiliares, criadas para designar os níveis das variáveis categóricas selecionadas. Para efeitos de comparação, considerou-se o modelo linear com intercepto aleatório para descrever a transformação logito dos índices de utilidade.

A descrição dos tempos de sobrevivência foi feita através do modelo de taxa de falhas proporcionais Weibull. Sua adequabilidade foi verificada considerando a análise dos resíduos de Cox-Snell (Klein e Moeschberger, 2003). A escolha de um modelo paramétrico para esta aplicação se deve à (i) uma conveniência computacional e (ii) pelo fato da abordagem semiparamétrica subestimar os erros padrão das estimativas em modelagens conjuntas (Hsieh et al., 2006). O modelo resultante do processo de seleção de variáveis é dado por

$$
\begin{aligned}
h_{i}\left(t \mid \mathcal{M}_{i}(t), \boldsymbol{\omega}_{i}\right) & =\rho t^{\rho-1} \cdot \exp \left\{\gamma_{0}+\gamma_{1} \text { ExtCancer-LA }_{i}+\gamma_{2} \text { ExtCancer-MD }_{i}\right. \\
& +\gamma_{3} \text { TipoAdm }_{i}+\gamma_{4} \text { Radio }_{i}+\gamma_{5} \text { Delirium-D }_{i} \\
& \left.+\gamma_{6} \text { Delirium-S }_{i}+\alpha m_{i}(t)\right\} .
\end{aligned}
$$

com ExtCancer-LA, ExtCancer, TipoAdm, Radio, Delirium-D e Delirium-S variáveis binárias auxiliares, que representam os níveis das variáveis categóricas selecionadas para o modelo de sobrevivência.

\subsection{Aspectos computacionais}

A estimação de máxima verossimilhança foi implementada em ambiente R (R Core Team, 2020), utilizando-se o algoritmo quase-Newton BFGS (Nocedal e Wright, 2006; Gill et al., 2019) do otimizador optim. Esse procedimento foi escolhido pelas suas propriedades de convergência local e por fazer um balanço entre ordem de convergência e custo computacional. As estimativas dos modelos marginais foram usadas na inicialização do processo iterativo. Para o parâmetro de associação, fixou-se um valor próximo de zero como chute inicial. Em todos os modelos ajustados, as iterações convergiram para um ponto de máximo.

Com relação à implementação, a aproximação da integral (4.12) através das regras de quadratura é feita por meio de sucessivos somatórios, que geram repetições de subrotinas dentro de laços aninhados. Por essa razão, optou-se pela vetorização de funções custosas para as iterações, reescrevendo as rotinas envolvidas no cálculo da verossimilhança marginal como um conjunto de operações de matrizes, que são 
Tabela 8.1: Desempenho do processo de estimação dos modelos conjuntos, considerando a reescala da verossimilhança através dos fatores determinados pelo vetor identidade, por valores típicos dos parâmetros e pelo precondicionador de Jacobi.

\begin{tabular}{clcccc}
\hline Modelos conjuntos & Fatores de escala & $\mathcal{N}_{1}$ & $\mathcal{N}_{2}$ & $\kappa(H(\hat{\boldsymbol{\theta}}))$ & $\ell(\hat{\boldsymbol{\theta}})$ \\
\hline \multirow{3}{*}{ BE - WEI } & Identidade & 695 & 119 & $10^{6}$ & $-449,208$ \\
& Valores típicos & 546 & 78 & $10^{3}$ & $-449,201$ \\
& Jacobi & $\mathbf{4 3 6}$ & $\mathbf{4 0}$ & $\mathbf{1 0}^{\mathbf{2}}$ & $\mathbf{- 4 4 9 , 2 0 1}$ \\
\hline \multirow{3}{*}{ BEI - WEI } & Identidade & 655 & 133 & $10^{7}$ & $-3508,391$ \\
& Valores típicos & 631 & 91 & $10^{3}$ & $-3508,390$ \\
& Jacobi & $\mathbf{4 3 6}$ & $\mathbf{4 0}$ & $\mathbf{1 0}^{\mathbf{2}}$ & $\mathbf{- 3 5 0 8 , 3 9 0}$ \\
\hline \multirow{3}{*}{ LN - WEI } & Identidade & 502 & 88 & $10^{6}$ & $-11209,728$ \\
& Valores típicos & 475 & 79 & $10^{4}$ & $-11209,728$ \\
& Jacobi & $\mathbf{2 8 9}$ & $\mathbf{1 9}$ & $\mathbf{1 0}$ & $\mathbf{- 1 1 2 0 9 , 7 2 8}$ \\
\hline
\end{tabular}

mais eficientes em linguagens interpretadas como o R. Os códigos com as estruturas de dados usadas para esse fim foram adaptados de funções do pacote JM (Rizopoulos, 2010) e sua aplicação no cálculo da verossimilhança marginal de um modelo conjunto, são encontrados no Apêndice C.

Na Tabela 8.1 estão dispostas as métricas $\mathcal{N}_{1}$ e $\mathcal{N}_{2}$ (especificadas no Capítulo 7), a ordem de magnitude do número de condição da matriz hessiana em $\hat{\boldsymbol{\theta}}$ e o valor de $\ell(\boldsymbol{\theta})$ na estimativa fornecida. Todas as medidas foram obtidas após o ajuste dos modelos BE-WEI, BEI-WEI e LN-WEI, considerando os fatores de escala definidos pelo vetor identidade, por valores típicos dos parâmetros, especificados pelas estimativas dos modelos marginais, e pelo precondicionador de Jacobi.

Observa-se que as reparametrizações propostas diminuíram o número de condição de $H(\hat{\boldsymbol{\theta}})$, melhorando a convergência do processo de estimação. Como consequência, (i) reduções significativas foram observadas nos números de avaliações da verossimilhança, o que é verificado pelas medidas $\mathcal{N}_{1}$ e $\mathcal{N}_{2}$ e (ii) comparativamente ao ajuste sem reescala e com o uso dos diferentes fatores propostos, uma pequena diferença é notada nos valores das log-verossimilhanças do modelo BEI-WEI e do modelo BE-WEI. Isso sinaliza que nas situações de mal condicionamento, os resultados fornecidos podem ter carregado mais os erros de aproximação numérica.

Outro aspecto positivo da melhora do condicionamento em abordagens frequentistas, é a correção da estabilidade dos procedimentos iterativos. Para ilustrar esse problema, considera-se o ajuste do modelo conjunto LN-WEI aos dados do ICESP, através do método BFGS disponível no pacote JM. A principal diferença entre essas rotinas e as implementadas neste trabalho está no cálculo do vetor escore para a 
Tabela 8.2: Desempenho do processo de estimação do modelo conjunto LN-WEI no pacote JM, considerando a reescala da verossimilhança através dos fatores determinados pelo vetor identidade, por valores típicos dos parâmetros e pelo precondicionador de Jacobi.

\begin{tabular}{clccc}
\hline Modelo conjunto & Fatores de escala & $\mathcal{N}_{\text {iter }}$ & $\kappa(H(\hat{\boldsymbol{\theta}}))$ & $\ell(\hat{\boldsymbol{\theta}})$ \\
\hline \multirow{3}{*}{ LN - WEI } & Valores típicos & 44 & $10^{3}$ & $-11215,396$ \\
& Jacobi & $\mathbf{1 9}$ & $\mathbf{1 0}$ & $\mathbf{- 1 1 2 1 5 , 3 8 6}$ \\
& Valores típicos sem critérios & 122 & $10^{6}$ & $-11215,573$ \\
& Identidade & $*$ & $*$ & $*$ \\
\hline
\end{tabular}

* Processo não convergente.

maximização de $\ell(\boldsymbol{\theta})$. No primeiro caso, a abordagem utilizada se baseia na aproximação de uma integral numérica (Rizopoulos, 2012), ao passo que no segundo é considerado o método das diferenças finitas. As estratégias mencionadas resultam em diferentes tipos de erros de aproximação, tornando a convergência do processo de estimação dependente dessas escolhas metodológicas.

Na Tabela 8.2 mostra-se a performance da estimação segundo o número de iterações $\left(\mathcal{N}_{\text {iter }}\right)$ do algoritmo BFGS ${ }^{1}$. A estimação apresentou uma falha de convergência quando não se empregou a correção do condicionamento, causada por instabilidades numéricas ${ }^{2}$. A aplicação das reescalas propostas mostrouse eficiente para o controle das imprecisões numéricas durante o processo iterativo. Também considerouse o uso de reescalas com valores típicos escolhidas sem critérios, simulando-se uma situação prática na qual um vetor de escalas é definido sem a análise de condicionamento. É possível perceber um aumento no número de iterações nesse caso, causando pela piora do condicionamento. Além disso, o aumento de $\kappa(H(\hat{\boldsymbol{\theta}}))$ modificou o valor da log-verossimilhança na estimativa de máxima verossimilhança, indicando a existência de possíveis imprecisões numéricas nos resultados obtidos.

\subsection{Estimação}

A Tabela 8.3 mostra as estimativas dos parâmetros e dos seus erros padrão, e o valor-p dos testes de Wald para os processos longitudinais, de acordo com os modelos BE-WEI, BEI-WEI e LN-WEI. Os parâmetros dos modelos de regressão beta e beta inflacionado são comparáveis, pois descrevem as trajetórias de escores de qualidade de vida (transformados e em escala original, respectivamente) que não assumem valores na fronteira do intervalo $(0,1]$.

\footnotetext{
${ }^{1}$ Essa escolha foi feita pois o número de iterações do quase-Newton é a única medida de desempenho do otimizador, disponibilizada no pacote JM.

${ }^{2} \mathrm{O}$ acompanhamento da convergência foi feito imprimindo-se, a cada iteração, os valores da sequência de parâmetros gerada pelo algoritmo BFGS. Verificou-se que ao final do processo, foram geradas aproximações cada vez maiores para os parâmetros, até a sua interrupção devido ao alto valor da log-verossimilhança
} 
Tabela 8.3: Estimativas para o processo longitudinal, considerando os modelos BE-WEI, BEI-WEI e LN-WEI.

\begin{tabular}{|c|c|c|c|c|}
\hline Modelo Conjunto & Parâmetros & Estimativas & Erros padrão & valor $\mathrm{p}$ \\
\hline \multirow{9}{*}{ BE-WEI } & Intercepto & 0,4530 & 0,0646 & $<0,0001$ \\
\hline & Tempo Obs. & 0,0008 & 0,0001 & $<0,0001$ \\
\hline & Radioterapia - Sim & $-0,2402$ & 0,0942 & 0,0108 \\
\hline & $E C O G-1$ & $-0,5635$ & 0,0900 & $<0,0001$ \\
\hline & $E C O G-2$ & $-0,9799$ & 0,1171 & $<0,0001$ \\
\hline & ECOG - 3 & $-1,6297$ & 0,1289 & $<0,0001$ \\
\hline & $E C O G-4$ & $-2,1479$ & 0,1923 & $<0,0001$ \\
\hline & $D$ & 0,6276 & 0,0921 & $<0,0001$ \\
\hline & $\phi$ & 1,1350 & 0,0286 & $<0,0001$ \\
\hline \multirow{10}{*}{ BEI-WEI } & Intercepto & $-0,2131$ & 0,0609 & $<0,0001$ \\
\hline & Tempo Obs. & 0,0006 & 0,0001 & $<0,0001$ \\
\hline & Radioterapia - Sim & $-0,1914$ & 0,0850 & 0,0244 \\
\hline & $E C O G-1$ & $-0,2853$ & 0,0826 & $<0,0001$ \\
\hline & $E C O G-2$ & $-0,6414$ & 0,1042 & $<0,0001$ \\
\hline & ECOG - 3 & $-1,3327$ & 0,1172 & $<0,0001$ \\
\hline & $E C O G-4$ & $-1,8193$ & 0,1745 & $<0,0001$ \\
\hline & $D$ & 0,4374 & 0,1122 & $<0,0001$ \\
\hline & $\phi$ & 1,7177 & 0,0321 & $<0,0001$ \\
\hline & $\operatorname{logito}(\xi)$ & $-1,4677$ & 0,0475 & $<0,0001$ \\
\hline \multirow{9}{*}{ LN-WEI } & Intercepto & 1,3006 & 0,1835 & $<0,0001$ \\
\hline & Tempo Obs. & 0,0023 & 0,0003 & $<0,0001$ \\
\hline & Radioterapia - Sim & $-0,6700$ & 0,2738 & 0,0140 \\
\hline & $E C O G-1$ & $-1,6353$ & 0,2637 & $<0,0001$ \\
\hline & $E C O G-2$ & $-2,7726$ & 0,3363 & $<0,0001$ \\
\hline & $E C O G-3$ & $-4,8523$ & 0,3722 & $<0,0001$ \\
\hline & $E C O G-4$ & $-6,8988$ & 0,5594 & $<0,0001$ \\
\hline & $D$ & 5,6186 & 0,0808 & $<0,0001$ \\
\hline & $\sigma$ & 3,3221 & 0,0150 & $<0,0001$ \\
\hline
\end{tabular}

Diferenças entre os resultados obtidos são observadas, embora o mesmo conjunto de parâmetros tenha sido identificado pelas duas abordagens para a significância estatística. Nos dois casos, a taxa de variação estimada (i) é positiva, indicando uma evolução crescente da resposta longitudinal, e (ii) maior para modelo de regressão beta, o que pode ser em razão da transformação das observações de um.

Com relação ao processo de sobrevivência, na Tabela 8.4 é possível verificar que todos os modelos identificaram uma associação negativa e significativa entre o risco de óbito e a evolução da qualidade de vida. Em termos de estimativas e erros padrão, as três abordagens forneceram resultados semelhantes para os fatores considerados. As abordagens diferem nos valores dos parâmetros de associação e da função de taxa de falha basal do modelo Weibull. Em particular, a acomodação dos escores máximos na modelagem pode ter captado melhor a associação entre as respostas, uma vez que o modelo BEI-WEI forneceu a maior estimativa para o parâmetro $\alpha$. Este fato mostra a importância de considerar a restrição das respostas longitudinais na descrição dos dados, sobretudo em cenários com proporções relevantes de 
Tabela 8.4: Estimativas para o processo de sobrevivência, considerando os modelos BE-WEI, BEI-WEI e LN-WEI.

\begin{tabular}{l|lrrr}
\hline Modelo Conjunto & Parâmetros & Estimativas & Erros padrão & valor p \\
\hline \multirow{5}{*}{ BE-WEI } & Intercepto & $-2,0706$ & 0,1880 & $<0,0001$ \\
& Extensão do Câncer - Localmente avançado & 0,2744 & 0,1210 & 0,0233 \\
& Extensão do Câncer - Metástase à distância & 0,5005 & 0,1231 & $<0,0001$ \\
& Tipo admissão UTI - Planejada & $-0,7916$ & 0,0979 & $<0,0001$ \\
& Radioterapia - Sim & 0,0851 & 0,1091 & 0,4356 \\
& Delirium - Sedado & 0,7552 & 0,1290 & $<0,0001$ \\
& Delirium - Sim & 0,1791 & 0,1248 & 0,1514 \\
& $\alpha$ & $-2,8144$ & 0,3155 & $<0,0001$ \\
& $\rho$ & $-0,5669$ & 0,0505 & $<0,0001$ \\
\hline \multirow{5}{*}{ BEI-WEI } & $-1,4305$ & 0,2457 & $<0,0001$ \\
& Intercepto & 0,3023 & 0,1212 & 0,0126 \\
& Extensão do Câncer - Localmente avançado & 0,5444 & 0,1231 & $<0,0001$ \\
& Extensão do Câncer - Metástase à distância & 0,090 & $<0,0001$ \\
& Tipo admissão UTI - Planejada & -0.7979 & 0,0980 \\
& Radioterapia - Sim & 0,0833 & 0,1097 & 0,4474 \\
& Delirium - Sedado & 0,7399 & 0,1279 & $<0,0001$ \\
& Delirium - Sim & 0,1877 & 0,1249 & 0,1330 \\
& $\alpha$ & $-3,8608$ & 0,5230 & $<0,0001$ \\
& $\rho$ & $-0,5998$ & 0,0520 & $<0,0001$ \\
\hline & Intercepto & $-3,4854$ & 0,2025 & $<0,0001$ \\
& Extensão do Câncer - Localmente avançado & 0,2800 & 0,1204 & 0,0200 \\
& Extensão do Câncer - Metástase à distância & 0,5071 & 0,1224 & $<0,0001$ \\
& Tipo admissão UTI - Planejada & $-0,7959$ & 0,0976 & $<0,0001$ \\
& Radioterapia - Sim & 0,0954 & 0,1082 & 0,3779 \\
& Delirium - Sedado & 0,7318 & 0,1282 & $<0,0001$ \\
& Delirium - Sim & 0,1628 & 0,1247 & 0,1918 \\
& $\alpha$ & $-0,1884$ & 0,0202 & $<0,0001$ \\
& $\rho$ & $-0,5618$ & 0,0497 & $<0,0001$ \\
\hline & $\rho$ & & &
\end{tabular}

pontos extremos.

\subsection{Predições de sobrevivência}

Os resultados discutidos na seção anterior sugerem que os escores de qualidade de vida, mensurados através do instrumento EQ-5D, estão negativamente associados à taxa de ocorrência de óbito. Essa informação, isoladamente, não esclarece de que maneira os índices de utilidade podem aperfeiçoar as predições das sobrevidas. Além disso, os modelos conjuntos têm uma capacidade limitada enquanto instrumentos de predição, de modo que é importante verificar a sua acurácia antes de empregá-los para este fim. Esta seção tem a finalidade de (I) investigar a habilidade preditiva das medições de qualidade de vida na sobrevida global e (II) avaliar os ganhos das abordagens propostas nas predições de sobrevivência individuais dos pacientes do ICESP. 


\subsubsection{Performance dos índices de utilidade como preditores de mortalidade}

Neste trabalho, a performance preditiva dos modelos utilizados para a análise dos tempos de sobrevivência é medida através do erro de predição $(E P)$. Essa métrica quantifica a perda esperada entre o status de sobrevivência observado e a probabilidade de sobrevivência predita em um instante $u>t$, dado que foram registradas informações longitudinais até o instante $t>0$, ou seja

$$
E P(u \mid t)=E\left(L\left(N_{i}(t)-\pi_{i}(u \mid t) \mid T_{i} \geq u\right)\right.
$$

em que $N_{i}(t)=I\left(T_{i} \geq t\right)$ denota o status do evento no instante $t, L(\cdot)$ representa uma função de perda e a esperança é tomada com relação aos tempos de sobrevivência. Uma estimativa para (8.3), que leva em conta as observações censuradas no intervalo $[t, u]$, é proposta em Henderson et al. (2002), e dada por

$$
\begin{aligned}
\widehat{E P}(u \mid t) & =\frac{1}{n(t)} \sum_{i: T i \geq t} I\left(T_{i} \geq u\right) L(1-\hat{\pi}(u \mid t))+\delta_{i} I\left(T_{i} \leq u\right) L(0-\hat{\pi}(u \mid t)) \\
& =\left(1-\delta_{i}\right) I\left(T_{i} \leq u\right) L(0-\hat{\pi}(u \mid t))\left[\hat{\pi}\left(u \mid T_{i}\right) L(1-\hat{\pi}(u \mid t))\right. \\
& \left.+\quad\left(1-\hat{\pi}\left(u \mid T_{i}\right)\right) L(0-\hat{\pi}(u \mid t))\right]
\end{aligned}
$$

em que $n(t)$ denota o número de indivíduos em risco no instante $t$. Os dois primeiros termos da soma correspondem, respectivamente, aos indivíduos que experimentaram o evento posterior e anteriormente ao instante $u$. A terceira parcela da expressão diz respeitos aos indivíduos que foram censurados no intervalo $[t, u]$. Para esta aplicação, considera-se a perda quadrática. Neste caso, erros de predição iguais a zero indicam a melhor capacidade preditiva possível e erros iguais a 0,25 são obtidos através de um modelo não informativo para a predição da sobrevivência, fixando-se $\hat{\pi}_{i}(u \mid t)=0,5$, para todo $i=1, \ldots, n$.

Para investigar a habilidade preditiva da qualidade de vida na mortalidade, as predições de sobrevivência foram obtidas por meio dos modelos conjuntos BEI-WEI, BE-WEI e LN-WEI, especificados como na Seção 8.1. O modelo de taxa de falhas proporcionais Weibull foi usado nessa análise como um modelo de referência, cuja formulação considerou as variáveis explicativas selecionadas na Seção 8.1 sem adicionar qualquer informação longitudinal referente aos índices de utilidade observados. 
A validação das predições foi feita em amostras diferentes da que foi realizada a estimação, a fim de se evitar o sobre-ajuste. Considerou-se, para tanto, o procedimento de validação cruzada. Mais especificamente, os dados originais foram divididos aleatoriamente em 10 partes. Cada subamostra foi permutada para a estimação dos modelos (amostra de treino) e para o cálculo do erro de predição (amostra de teste). Esse processo foi repetido por 20 vezes para cada tempo de coleta $t$ das medidas longitudinais e em diferentes pontos $u=t+\Delta t$, o que gerou as curvas com as estimativas dos erros de predição disponíveis na Figura 8.1.

Observa-se que a incorporação das trajetórias individuais de qualidade de vida nos modelos de sobrevivência ajudou a explicar melhor a heterogeneidade dos pacientes. Isto é especialmente notado no início do acompanhamento, período no qual os erros de predição associados aos modelos conjuntos são menores comparados ao modelo de referência Weibull. No entanto, em termos de EPs, a precisão das predições fornecidas pela abordagem marginal e conjunta vai se equiparando conforme mais informações longitudinais são obtidas. Esse fato pode estar relacionado ao comportamento da amostra de sobreviventes desse estudo. Quanto maior o afastamento da admissão nas UTIs, maiores são as magnitude e estabilidade observadas nas respostas longitudinais de qualidade de vida (Normilio-Silva et al., 2016). Sem grandes diferenças entre as respostas longitudinais dos pacientes, menor a capacidade dessas medidas em discriminá-los em uma análise preditiva.

\subsubsection{Estudo de casos com predições de sobrevidas individuais}

O erro de predição fornece a acurácia média de um modelo preditivo na população de interesse, o que não permite avaliar seu desempenho na estimação das sobrevidas individuais. Esta seção analisa como as metodologias propostas acomodam perfis longitudinais problemáticos, como os descritos no Capítulo 2, e contribuem para o aprimoramento das predições de sobrevivência. Com esse objetivo, um estudo de casos é apresentado considerando a predição das sobrevidas individuais de pacientes selecionados aleatoriamente do conjunto de dados do ICESP.

A Figura 8.2 mostra os escores de qualidade de vida observados e as curvas de sobrevivência preditas segundo os modelos BEI-WEI, BE-WEI e LN-WEI, para nove participantes do estudo. Esses indivíduos, que encontram-se identificados na Tabela 8.5, foram removidos da amostra antes do processo de estimação, a fim de se evitar o sobreajuste. 
Erro predição condicional, $t=15$

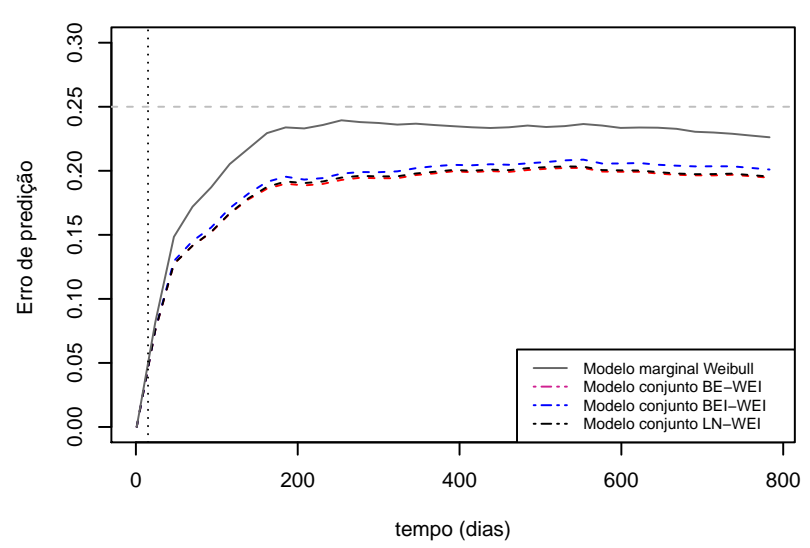

Erro predição condicional, $t=180$

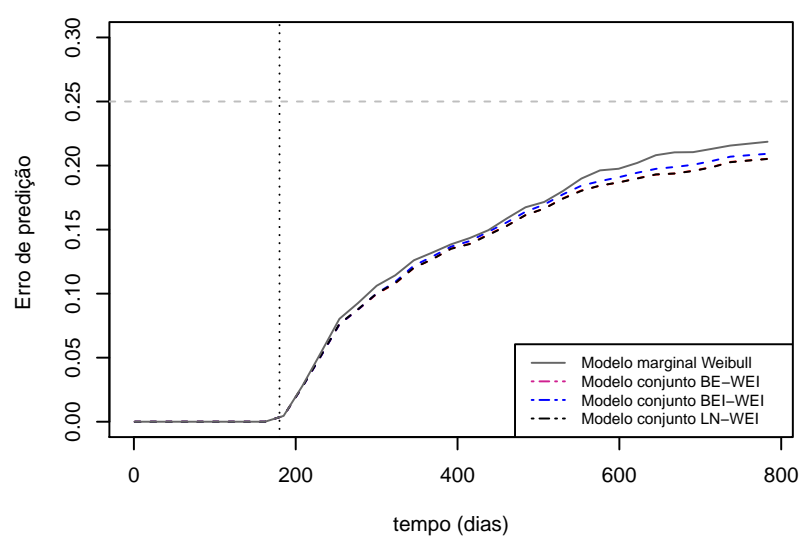

Erro predição condicional, $\mathrm{t}=\mathbf{5 4 0}$

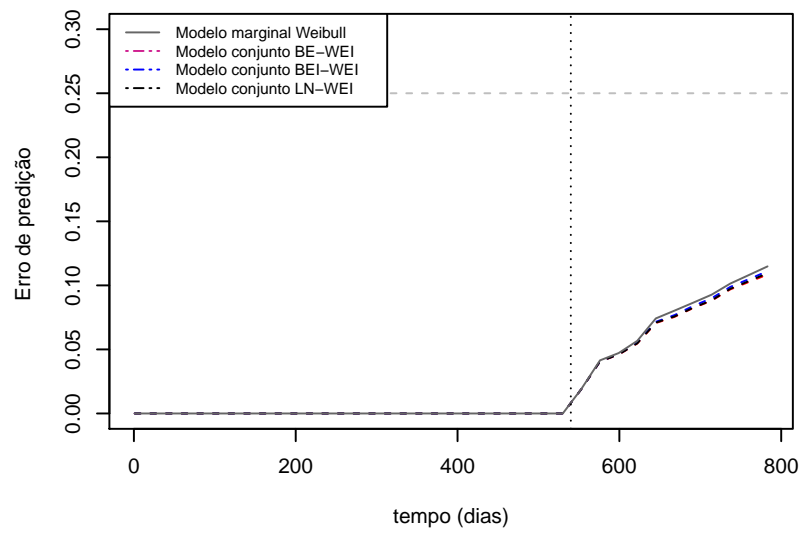

Erro predição condicional, $\mathrm{t}=90$

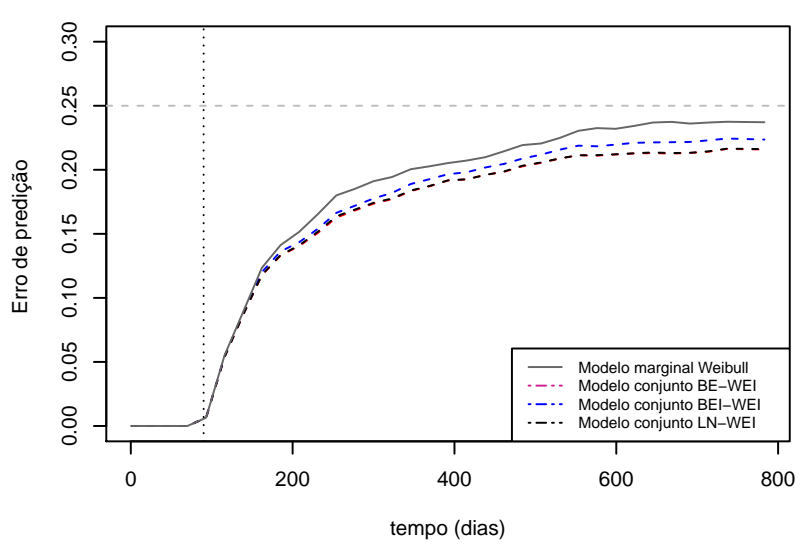

Erro predição condicional, $\mathrm{t}=360$

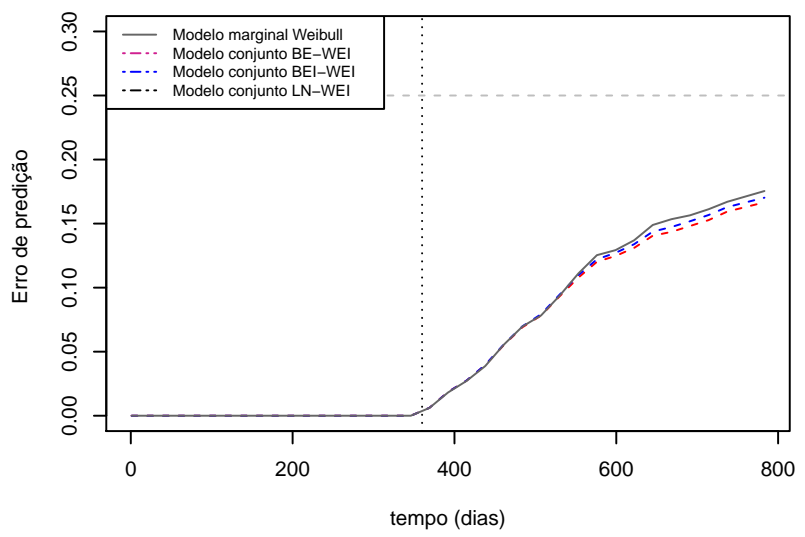

Figura 8.1: Estimativas para os erros de predição das probabilidades de sobrevivência condicionais $\hat{\pi}(u \mid t)$, com $t=15,90,180,360,540)$, obtidas através de 20 repetições do procedimento de validação cruzada. 
Tabela 8.5: Perfil dos pacientes selecionados segundo as variáveis explicativas radioterapia prévia, delirium, ECOG, extensão do câncer e tipo admissão UTI; e respostas de sobrevivência observadas.

\begin{tabular}{c|ccccccc}
\hline & \multicolumn{5}{c}{ Variáveis explicativas } & & \\
Paciente & Radio prévia & $E C O G$ & Delirium & Extensão câncer & Tipo adm.UTI & $\delta_{i}$ & $T_{i}$ \\
\hline 7 & Não & 1 & Sim & Limitado & Planejada & 0 & 729 \\
\hline 14 & Não & 3 & Não & Metástase a distância & Não planejada & 1 & 167 \\
37 & Não & 0 & Não & Localmente avançado & Não planejada & 1 & 213 \\
165 & Não & 4 & Não & Limitado & Planejada & 0 & 762 \\
179 & Sim & 0 & Sedado & Localmente avancado & Planejada & 1 & 120 \\
349 & Não & 0 & Não & Localmente avancado & Planejada & 1 & 383 \\
441 & Não & 3 & Não & Metástase a distância & Planejada & 1 & 567 \\
570 & Não & 0 & Não & Metástase a distância & Não planejada & 0 & 738 \\
\hline 585 & Não & 2 & Não & Localmente avançado & Planejada & 0 & 736 \\
654 & Não & 2 & Não & Limitado & Planejada & 1 & 324 \\
667 & Não & 4 & Sim & Limitado & Planejada & 1 & 617 \\
685 & Não & 1 & Não & Localmente avançado & Planejada & 0 & 723 \\
776 & Não & 4 & Não & Localmente avançado & Planejada & 1 & 663 \\
\hline
\end{tabular}

Nota-se que para os pacientes $7, \mathbf{1 4}, 570$ e 685 , cujos escores descreveram trajetórias longitudinais estáveis nos períodos que antecederam o último tempo de coleta dos dados, ou cujas respostas longitudinais observadas permaneceram no intervalo aberto $(0,1)$, os modelos conjuntos forneceram predições de sobrevidas semelhantes. Já os indivíduos 667 e 776 foram acompanhados até o final do estudo e durante todo esse período seus estados de saúde permaneceram comprometidos. Nesses casos, o modelo BEI-WEI forneceu predições mais otimistas de sobrevivência. Isso ocorre pois a especificação da média longitudinal do modelo inflacionado que é inserida no modelo de sobrevivência acomoda efeitos populacionais que podem descrever o aumento da qualidade de vida e e o prolongamento da sobrevida de pacientes graves, como é explicado na Seção 4.4 .

Os pacientes 349, 179 e 654 apresentaram maiores variações entre as suas últimas medições de qualidade de vida. Além disso, embora esses indivíduos tenham ido a óbito antes da conclusão do estudo, nos três casos analisados houveram ocorrências de observações de escores máximos. É possível que essas observações de uns tenham minimizado o impacto de medições subsequentes mais baixas na atualização dos efeitos aleatórios. Como discutido na Seção 4.4, o fato do modelo inflacionado não considerar as respostas unitárias na predição de $\hat{\boldsymbol{b}}_{i}^{(t)}$ pode o ter favorecido na identificação da piora dos estados de saúde desses pacientes. Como consequência, predições de sobrevida individuais mais baixas foram fornecidas pelo modelo BEI-WEI nesses casos. 
Com o intuito de descrever mais detalhadamente a performance dos modelos considerados, uma análise das predições dinâmicas de sobrevivência dos pacientes 441,37 e 165 , é feita a seguir. Para tanto, considera-se a predição das sobrevidas condicionais em cada tempo de coleta das observações longitudinais, avaliando-se os seus valores preditos e as estimativas bootstrap para os seus erros padrão. Verifica-se que:

- O conjunto de índices de utilidades fornecidos pelo paciente 441 varia no intervalo $(0,1)$, como pode ser observado na Figura 8.3. Ao se utilizar estas medidas para as atualizações das sobrevidas condicionais, os três modelos considerados geraram predições semelhantes em todos os períodos nos quais o acompanhamento foi possível, como mostram a Figura 8.3 e a Tabela 8.7. A diferença entre as abordagens é dada pelos erros padrão das predições do modelo LN-WEI, que são maiores comparados aos dos modelos BE-WEI e BEI-WEI.

- O paciente 37 forneceu escores máximos em $t=15$ e $t=90$. As atualizações das predições das sobrevidas condicionais, bem como os valores dos seus erros padrão bootstrap, estão dispostos na Figura 8.4 e na Tabela 8.8. Verifica-se que as utilidades máximas influenciaram as predições de sobrevivência nos modelos BE-WEI e LN-WEI, o que gerou estimativas otimistas para a sua sobrevida, especialmente na atualização feita em $t=180$. Já o modelo BEI-WEI mostrou-se mais robusto às ocorrências de uns, fornecendo predições mais coerentes com o processo observado. Com relação aos erros padrão, os valores obtidos pelos modelos BE-WEI e BEI-WEI são semelhantes, enquanto o modelo LN-WEI forneceu predições com erros padrão maiores.

- O paciente 165 gerou escores de qualidade de vida próximas de zero durante todo o seguimento longitudinal, permanecendo vivo até o final do estudo $\left(T_{i} \geq 720\right)$. Em todos os períodos de coleta, as atualizações para a sua sobrevida são maiores quando se utiliza o modelo longitudinal inflacionado, como é mostrado na Figura 8.5 e na Tabela 8.9. Além disso, os erros padrão das predições foram menores comparados aos dos modelos BE-WEI e LN-WEI.

- Em todos os casos descritos, e para todos os modelos conjuntos considerados, nota-se que os erros padrão das predições diminuem na medida que novos escores de qualidade de vida são coletados. Isso reforça a vantagem da modelagem conjunta no estudo do ICESP, mostrando que o uso dessas informações longitudinais pode aumentar o grau de precisão das sobrevidas individuais estimadas. 
Tabela 8.6: Predições de sobrevivência condicionais para os pacientes 441, 37 e 165 após a coleta da medição $y_{180}$, em $u=t+\Delta t$, com $\Delta t=30,60,90$ e 180 .

\begin{tabular}{|c|c|c|c|c|c|c|c|c|c|}
\hline \multirow[b]{3}{*}{ Paciente } & \multirow{2}{*}{\multicolumn{3}{|c|}{ Respostas observadas }} & \multicolumn{6}{|c|}{ Predições } \\
\hline & & & & \multicolumn{2}{|c|}{$\mathrm{BE}-\mathrm{WEI}$} & \multicolumn{2}{|c|}{ BEI - WEI } & \multicolumn{2}{|c|}{ LN - WEI } \\
\hline & $T_{i}$ & $\delta_{i}$ & $y_{180}$ & $\Delta t$ & $\hat{\pi}(u \mid t)$ & $\Delta t$ & $\hat{\pi}(u \mid t)$ & $\Delta t$ & $\hat{\pi}(u \mid t)$ \\
\hline \multirow{4}{*}{441} & & & & 30 & 0,9369 & 30 & 0,9382 & 30 & 0,9447 \\
\hline & 567 & 1 & 0,0002 & 60 & 0,8820 & 60 & 0,8843 & 60 & 0,8962 \\
\hline & & & & 90 & 0,8337 & 90 & 0,8366 & 90 & 0,8527 \\
\hline & & & & 180 & 0,7173 & 180 & 0,7215 & 180 & 0,7469 \\
\hline \multirow{4}{*}{37} & & & & 30 & 0,9609 & 30 & 0,9261 & 30 & 0,9540 \\
\hline & 213 & 1 & 0,0004 & 60 & 0,9260 & 60 & 0,8626 & 60 & 0,9133 \\
\hline & & & & 90 & 0,8945 & 90 & 0,8074 & 90 & 0,8765 \\
\hline & & & & 180 & 0,8156 & 180 & 0,6771 & 180 & 0,7853 \\
\hline \multirow{4}{*}{165} & & & & 30 & 0,9399 & 30 & 0,9472 & 30 & 0,9244 \\
\hline & 762 & 0 & 0,0003 & 60 & 0,8873 & 60 & 0,9006 & 60 & 0,8596 \\
\hline & & & & 90 & 0,8405 & 90 & 0,8588 & 90 & 0,8028 \\
\hline & & & & 180 & 0,7262 & 180 & 0,7555 & 180 & 0,6692 \\
\hline
\end{tabular}

Na Tabela 8.6 comparam-se as predições de sobrevida para os pacientes 441,37 e 165 após a coleta da medição $y_{180}$, destacando-se em azul os valores que mais se aproximaram do processo observado. De um modo geral, nota-se que o modelo BEI-WEI discriminou melhor os pacientes para os quais o óbito foi observado, fornecendo menores probabilidades de sobrevivência apacientes que morreram mais cedo. Também destaca-se sua capacidade em captar o prolongamento da sobrevida em pacientes graves. O modelo BE-WEI teve boa habilidade discriminativa nos casos analisados quando não houve ocorrência de escore máximo. Por fim, embora o modelo LN-WEI forneça predições pontuais semelhantes aos modelos para dados limitados em alguns casos da amostra, sua performance na classificação de pacientes em riscos foi inferior aos outros. 

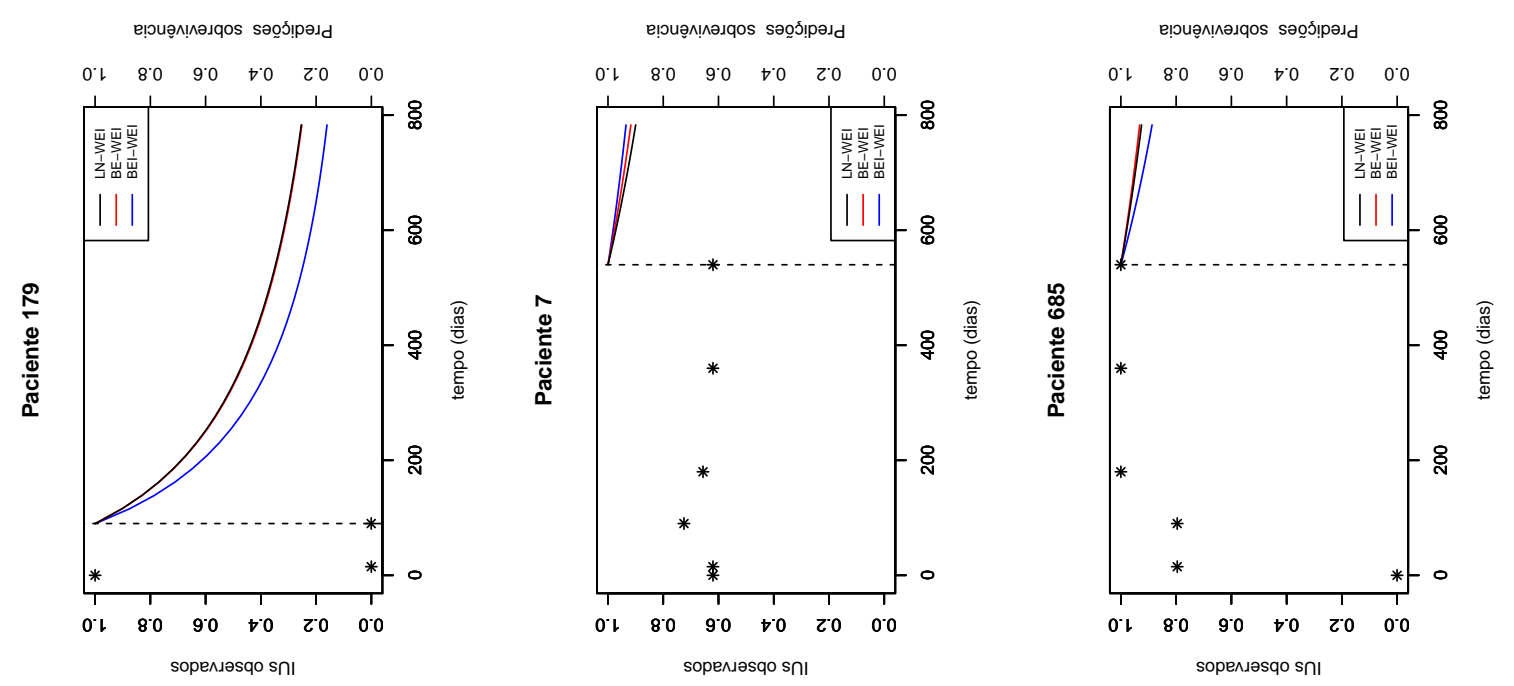

19
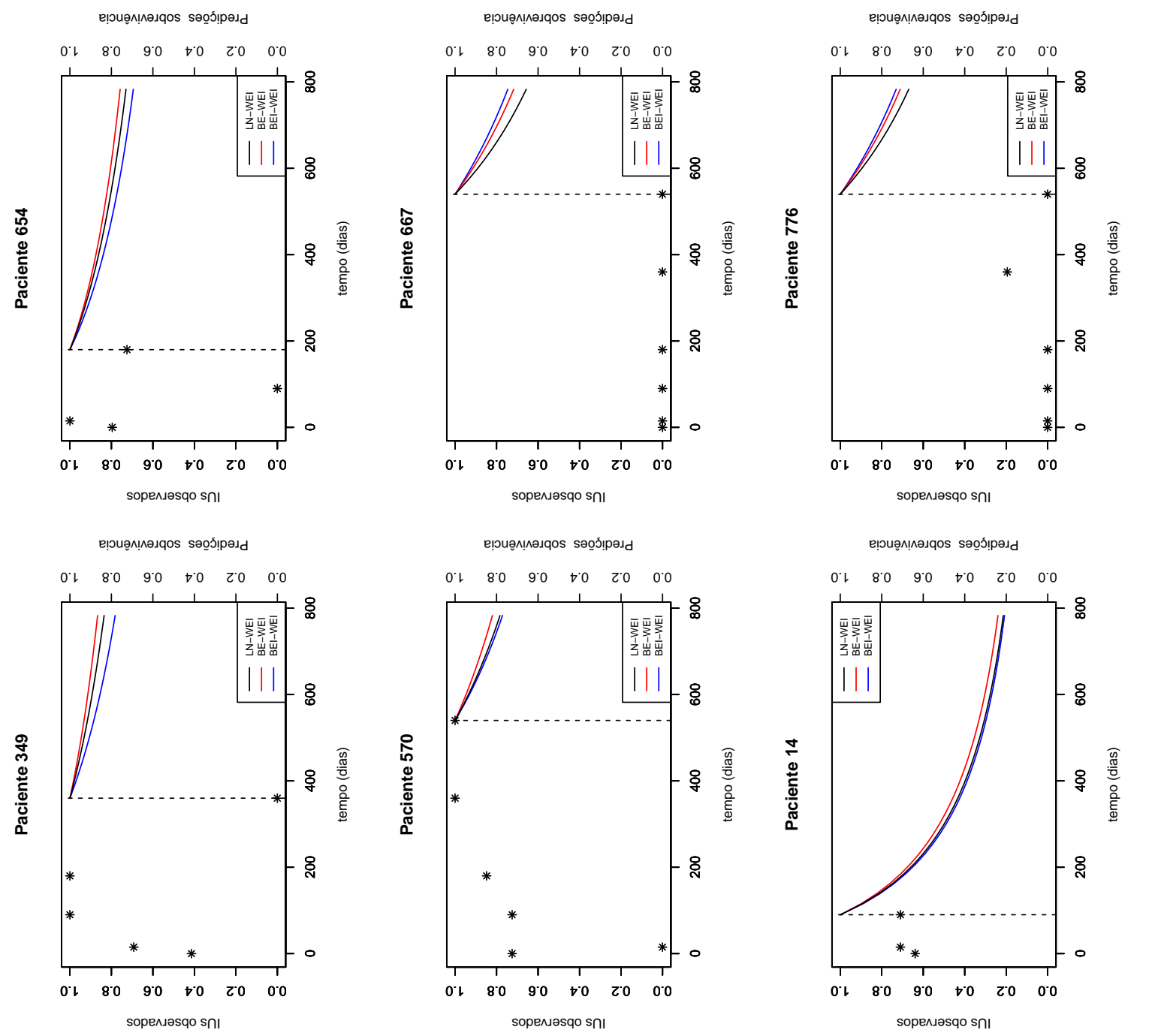

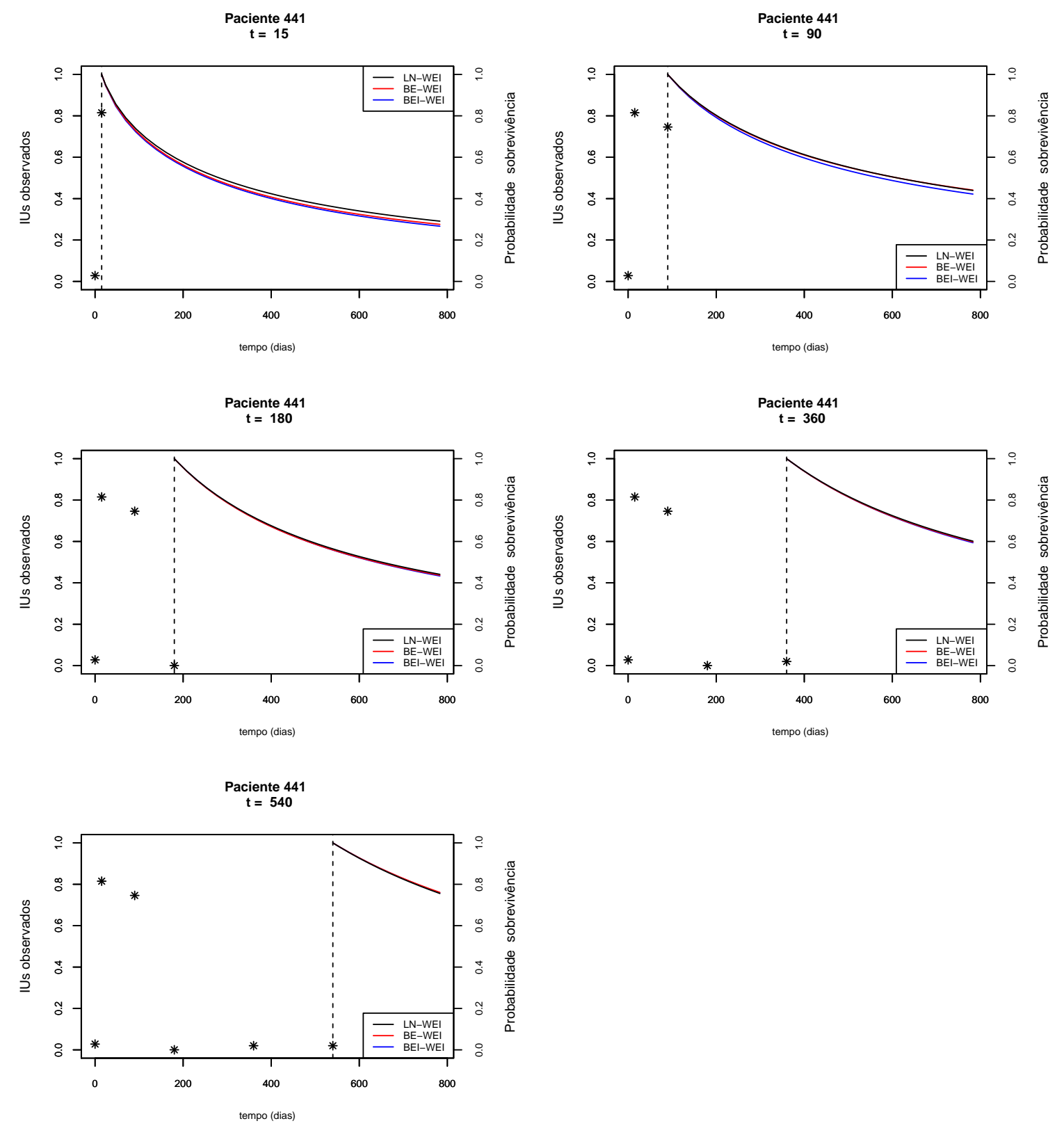

Figura 8.3: Índices de utilidade (IUs) observados e predições das sobrevidas condicionais para o paciente 441, segundo os modelos conjuntos BE-WEI e BEI-WEI e LN-WEI. 

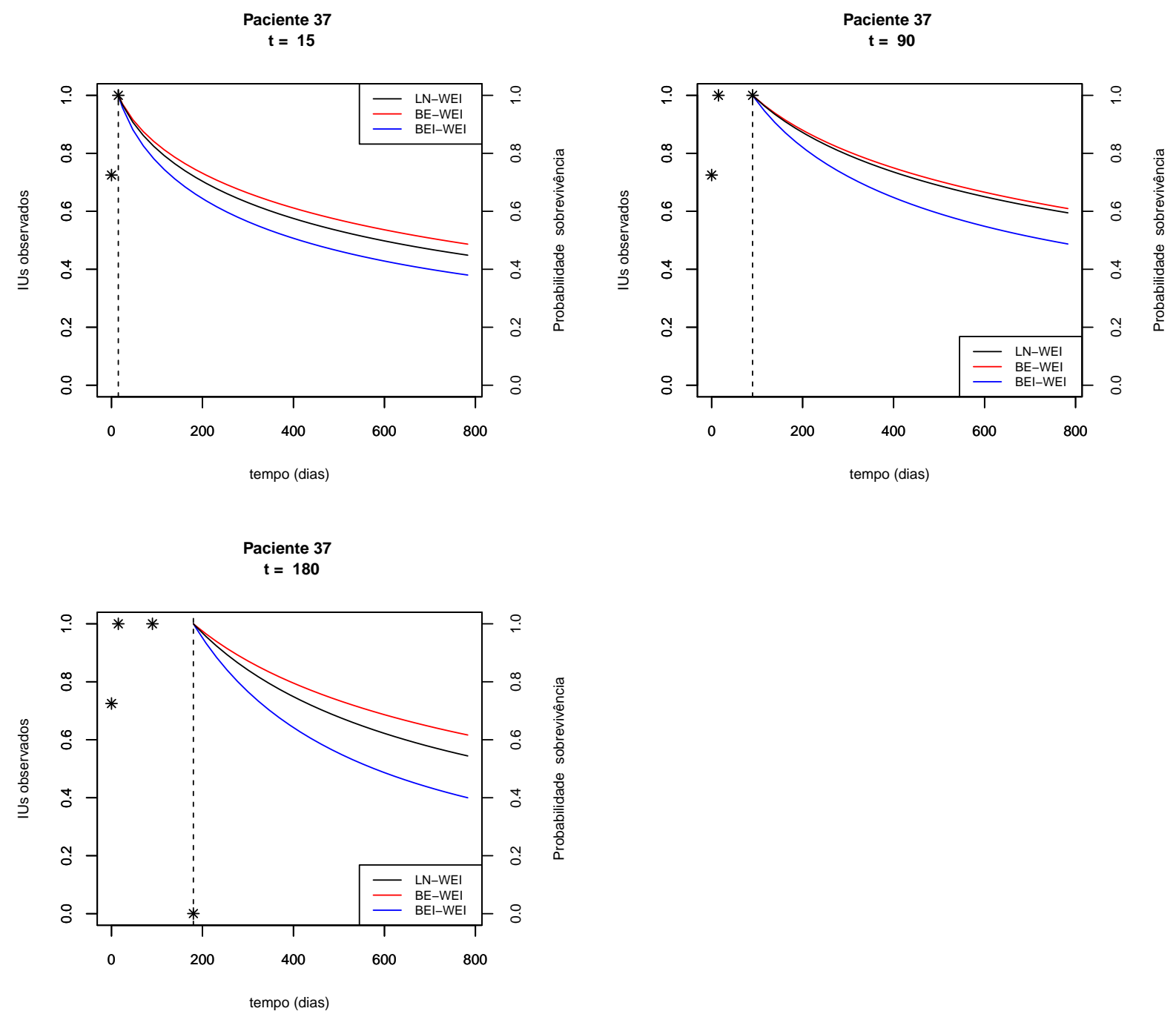

Figura 8.4: Índices de utilidade (IUs) observados e predições das sobrevidas condicionais para o paciente 37 , segundo os modelos conjuntos BE-WEI e BEI-WEI e LN-WEI. 

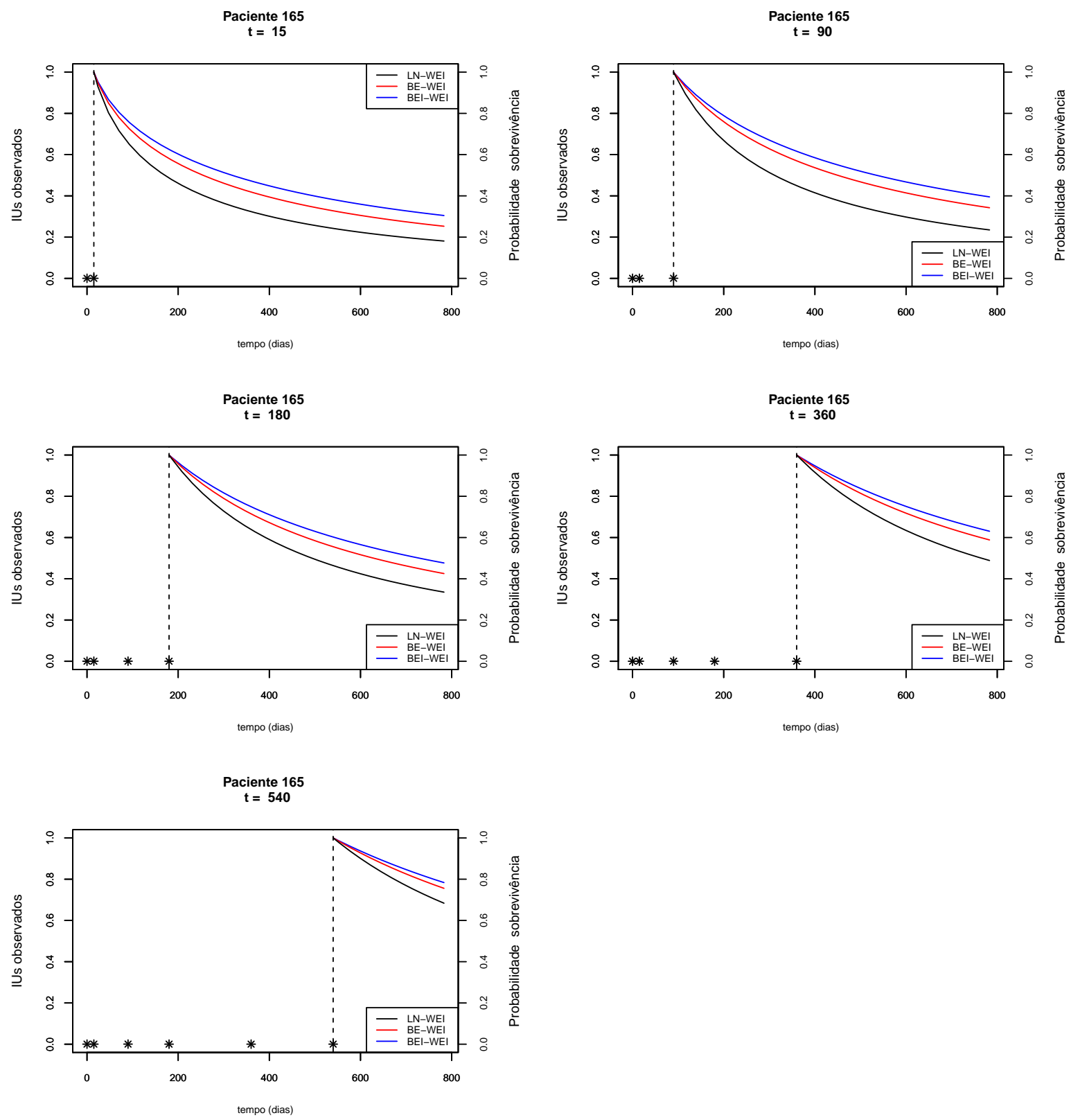

Figura 8.5: Índices de utilidade (IUs) observados e predições das sobrevidas condicionais para o paciente 165, segundo os modelos conjuntos BE-WEI e BEI-WEI e LN-WEI. 
Tabela 8.7: Predições de sobrevivência condicionais do paciente 441 após a inclusão da medida longitudinal $y_{t}$ ( $t=$ $15,90,180,360,540)$ e estimativas bootstrap para o seus respectivos erros padrão $\left(\widehat{E P}_{B}\right)$ em $u=t+\Delta t$, com $\Delta t=$ $30,60,90$ e 180 .

\begin{tabular}{c|ccc|ccc|ccc}
\hline Última medição & \multicolumn{3}{|c|}{$\mathrm{BE}-\mathrm{WEI}$} & \multicolumn{3}{c|}{$\mathrm{BEI}-\mathrm{WEI}$} & \multicolumn{3}{c}{ LN-WEI } \\
longitudinal & $\Delta t$ & $\hat{\pi}(u \mid t)$ & $\widehat{E P}_{B}$ & $\Delta t$ & $\hat{\pi}(u \mid t)$ & $\widehat{E P}_{B}$ & $\Delta t$ & $\hat{\pi}(u \mid t)$ & $\widehat{E P}_{B}$ \\
\hline \multirow{4}{*}{$y_{15}=0,8560$} & 30 & 0,8616 & 0,0115 & 30 & 0,8618 & 0,0122 & 30 & 0,8784 & 0,0336 \\
& 60 & 0,7743 & 0,0178 & 60 & 0,7754 & 0,0189 & 60 & 0,7988 & 0,0515 \\
& 90 & 0,7091 & 0,0219 & 90 & 0,7110 & 0,0233 & 90 & 0,7385 & 0,0630 \\
& 180 & 0,5761 & 0,0287 & 180 & 0,5797 & 0,0305 & 180 & 0,6126 & 0,0819 \\
\hline \multirow{5}{*}{$y_{90}=0,7460$} & 30 & 0,9324 & 0,0055 & 30 & 0,9327 & 0,0060 & 30 & 0,9362 & 0,0239 \\
& 60 & 0,8766 & 0,0098 & 60 & 0,8772 & 0,0106 & 60 & 0,8829 & 0,0421 \\
& 90 & 0,8291 & 0,0132 & 90 & 0,8299 & 0,0143 & 90 & 0,8370 & 0,0565 \\
$y_{180}=0,0002$ & 180 & 0,7190 & 0,0203 & 180 & 0,7203 & 0,0220 & 180 & 0,7302 & 0,0845 \\
\hline & 30 & 0,9369 & 0,0053 & 30 & 0,9382 & 0,0059 & 30 & 0,9447 & 0,0190 \\
& 60 & 0,8820 & 0,0097 & 60 & 0,8843 & 0,0108 & 60 & 0,8962 & 0,0340 \\
& 90 & 0,8337 & 0,0134 & 90 & 0,8366 & 0,0149 & 90 & 0,8527 & 0,0462 \\
& 180 & 0,7173 & 0,0211 & 180 & 0,7215 & 0,0236 & 180 & 0,7469 & 0,0709 \\
\hline \multirow{5}{*}{$y_{360}=0,0200$} & 30 & 0,9540 & 0,0039 & 30 & 0,9545 & 0,0045 & 30 & 0,9590 & 0,0109 \\
& 60 & 0,9121 & 0,0074 & 60 & 0,9131 & 0,0085 & 60 & 0,9214 & 0,0202 \\
& 90 & 0,8739 & 0,0104 & 90 & 0,8750 & 0,0120 & 90 & 0,8868 & 0,0285 \\
& 180 & 0,7765 & 0,0175 & 180 & 0,7778 & 0,0202 & 180 & 0,7975 & 0,0477 \\
\hline \multirow{3}{*}{$y_{540}=0,0200$} & 30 & 0,9625 & 0,0033 & 30 & 0,9627 & 0,0038 & 30 & 0,9649 & 0,0101 \\
& 60 & 0,9277 & 0,0062 & 60 & 0,9280 & 0,0073 & 60 & 0,9321 & 0,0193 \\
& 90 & 0,8954 & 0,0089 & 90 & 0,8956 & 0,0104 & 90 & 0,9016 & 0,0272 \\
& 180 & 0,8109 & 0,0153 & 180 & 0,8105 & 0,0181 & 180 & 0,8209 & 0,0465 \\
\hline
\end{tabular}

Tabela 8.8: Predições de sobrevivência condicionais do paciente 37 após a inclusão da medida longitudinal $y_{t}$ ( $t=$ $15,90,180)$ e estimativas bootstrap para o seus respectivos erros padrão $\left(\widehat{E P}_{B}\right)$ em $u=t+\Delta t$, com $\Delta t=30,60,90$ e 180 .

\begin{tabular}{|c|c|c|c|c|c|c|c|c|c|}
\hline \multirow{2}{*}{$\begin{array}{c}\text { Última medição } \\
\text { longitudinal }\end{array}$} & \multicolumn{3}{|c|}{ BE-WEI } & \multicolumn{3}{|c|}{ BEI-WEI } & \multicolumn{3}{|c|}{ LN-WEI } \\
\hline & $\Delta t$ & $\hat{\pi}(u \mid t)$ & $\widehat{E P}_{B}$ & $\Delta t$ & $\hat{\pi}(u \mid t)$ & $\widehat{E P}_{B}$ & $\Delta t$ & $\hat{\pi}(u \mid t)$ & $\widehat{E P}_{B}$ \\
\hline \multirow{4}{*}{$y_{15}=1,0000$} & 30 & 0,9170 & 0,0091 & 30 & 0,8875 & 0,0103 & 30 & 0,9105 & 0,0353 \\
\hline & 60 & 0,8618 & 0,0143 & 60 & 0,8157 & 0,0159 & 60 & 0,8501 & 0,0599 \\
\hline & 90 & 0,8187 & 0,0181 & 90 & 0,7613 & 0,0197 & 90 & 0,8032 & 0,0743 \\
\hline & 180 & 0,7251 & 0,0253 & 180 & 0,6479 & 0,0263 & 180 & 0,7017 & 0,0995 \\
\hline \multirow{4}{*}{$y_{90}=1,0000$} & 30 & 0,9584 & 0,0046 & 30 & 0,9387 & 0,0055 & 30 & 0,9555 & 0,0140 \\
\hline & 60 & 0,9230 & 0,0084 & 60 & 0,8880 & 0,0098 & 60 & 0,9175 & 0,0252 \\
\hline & 90 & 0,8921 & 0,0116 & 90 & 0,8447 & 0,0133 & 90 & 0,8842 & 0,0345 \\
\hline & 180 & 0,8169 & 0,0188 & 180 & 0,7437 & 0,0206 & 180 & 0,8042 & 0,0544 \\
\hline \multirow{4}{*}{$y_{180}=0,0004$} & 30 & 0,9609 & 0,0037 & 30 & 0,9261 & 0,0062 & 30 & 0,9540 & 0,0121 \\
\hline & 60 & 0,9260 & 0,0068 & 60 & 0,8626 & 0,0112 & 60 & 0,9133 & 0,0221 \\
\hline & 90 & 0,8945 & 0,0096 & 90 & 0,8074 & 0,0153 & 90 & 0,8765 & 0,0307 \\
\hline & 180 & 0,8156 & 0,0161 & 180 & 0,6771 & 0,0238 & 180 & 0,7853 & 0,0495 \\
\hline
\end{tabular}


Tabela 8.9: Predições de sobrevivência condicionais do paciente 165 após a inclusão da medida longitudinal $y_{t}(t=$ $15,90,180,360,540)$ e estimativas bootstrap para o seus respectivos erros padrão $\left(\widehat{E P}_{B}\right)$ em $u=t+\Delta t$, com $\Delta t=$ 30, 60, 90 e 180 .

\begin{tabular}{c|ccc|ccc|ccc}
\hline $\begin{array}{c}\text { Última medição } \\
\text { longitudinal }\end{array}$ & \multicolumn{3}{|c|}{$\mathrm{BE}-\mathrm{WEI}$} & $\hat{\pi}(u \mid t)$ & $\widehat{E P}_{B}$ & $\Delta t$ & $\hat{\pi}(u \mid t)$ & $\widehat{E P P}_{B}$ & \multicolumn{3}{c}{ LN-WEI } & $\hat{\pi}(u \mid t)$ & $\widehat{E P}_{B}$ \\
\hline \multirow{4}{*}{$y_{15}=0,0003$} & 30 & 0,8651 & 0,0148 & 30 & 0,8789 & 0,0138 & 30 & 0,8438 & 0,0542 \\
& 60 & 0,7793 & 0,0235 & 60 & 0,8016 & 0,0220 & 60 & 0,7453 & 0,0816 \\
& 90 & 0,7146 & 0,0295 & 90 & 0,7429 & 0,0277 & 90 & 0,6729 & 0,0979 \\
& 180 & 0,5810 & 0,0398 & 180 & 0,6200 & 0,0382 & 180 & 0,5283 & 0,1168 \\
\hline \multirow{5}{*}{$y_{90}=0,0005$} & 30 & 0,9216 & 0,0097 & 30 & 0,9308 & 0,0088 & 30 & 0,9083 & 0,0296 \\
& 60 & 0,8572 & 0,0171 & 60 & 0,8734 & 0,0158 & 60 & 0,8340 & 0,0507 \\
& 90 & 0,8025 & 0,0231 & 90 & 0,8243 & 0,0214 & 90 & 0,7715 & 0,0663 \\
$y_{180}=0,0003$ & 180 & 0,6765 & 0,0351 & 180 & 0,7096 & 0,0332 & 180 & 0,6328 & 0,0923 \\
\hline & 30 & 0,9399 & 0,0078 & 30 & 0,9472 & 0,0071 & 30 & 0,9244 & 0,0269 \\
& 60 & 0,8873 & 0,0143 & 60 & 0,9006 & 0,0131 & 60 & 0,8596 & 0,0471 \\
& 90 & 0,8405 & 0,0198 & 90 & 0,8588 & 0,0183 & 90 & 0,8028 & 0,0628 \\
& 180 & 0,7262 & 0,0318 & 180 & 0,7555 & 0,0299 & 180 & 0,6692 & 0,0919 \\
\hline \multirow{5}{*}{$y_{360}=0,0002$} & 30 & 0,9557 & 0,0060 & 30 & 0,9610 & 0,0055 & 30 & 0,9446 & 0,0158 \\
& 60 & 0,9151 & 0,0113 & 60 & 0,9250 & 0,0104 & 60 & 0,8944 & 0,0290 \\
& 90 & 0,8777 & 0,0160 & 90 & 0,8916 & 0,0148 & 90 & 0,8492 & 0,0400 \\
& 180 & 0,7813 & 0,0271 & 180 & 0,8046 & 0,0255 & 180 & 0,7355 & 0,0637 \\
\hline \multirow{5}{*}{$y_{540}=0,0003$} & 30 & 0,9636 & 0,0050 & 30 & 0,9678 & 0,0047 & 30 & 0,9541 & 0,0180 \\
& 60 & 0,9296 & 0,0096 & 60 & 0,9375 & 0,0089 & 60 & 0,9116 & 0,0336 \\
& 90 & 0,8978 & 0,0138 & 90 & 0,9090 & 0,0129 & 90 & 0,8728 & 0,0466 \\
& 180 & 0,8137 & 0,0240 & 180 & 0,8328 & 0,0227 & 180 & 0,7722 & 0,0753 \\
\hline
\end{tabular}




\section{Capítulo 9}

\section{Discussão e propostas}

Os modelos especificados nesta tese possibilitam a análise simultânea de respostas longitudinais restritas ao intervalo unitário e tempos de sobrevivência. Mais especificamente, considerou-se a investigação da associação dos índices de utilidade da qualidade de vida, medidos através do instrumento EQ-5D, e dos tempos de vida dos pacientes oncológicos do estudo do ICESP. Verificou-se que, ao se negligenciar o suporte dos dados longitudinais, a estimação da associação entre as respostas é comprometida, principalmente quando existe uma proporção relevante de observações de escores máximos na amostra.

Com relação ao estudo das predições de sobrevivência, o modelo BEI-WEI forneceu estimativas mais consistentes quando as observações de escores máximos não constituem valores genuínos da trajetória longitudinal do paciente. Quando os dados analisados possuem baixas proporções de uns, ou a condição de saúde da população investigada evolui verdadeiramente até atingir um patamar máximo, o modelo BE-WEI pode fornecer predições satisfatórias. Outro aspecto acomodado pela abordagem proposta refere-se às predições de sobrevida de pacientes com estados de saúde comprometidos (com sucessivos índices de utilidade próximos de zero) e tempos de vida elevados. Nestes cenários, o modelo BEI-WEI gerou as predições mais otimistas para a sobrevivência. Em todos os casos investigados, o modelo LN-WEI apresentou o pior desempenho em termos de estimativas e erros padrão para as probabilidades de sobrevivência, mostrando a importância da modelagem adequada da componente longitudinal em análises preditivas.

O presente trabalho também enfatizou a importância da escolha criteriosa dos procedimentos numéricos, com base em propriedades como robustez, precisão, estabilidade e eficiência. Uma análise dos problemas de condicionamento enfrentados na maximização da verossimilhança, no contexto da modelagem conjunta, foi realizada com o objetivo de identificar formas de melhorar suas características 
de convergência. A correção da sensibilidade da verossimilhança foi proposta através da reescala dos parâmetros, baseados em estratégias de precondicionamento. A aplicação da metodologia proposta permitiu concluir que este pode ser um caminho para a atenuação das falhas de convergência e do esforço computacional exigido em problemas complexos. É importante destacar que, até a conclusão deste trabalho, não foram encontradas abordagens voltadas para o estudo do condicionamento de procedimentos frequentistas. Em geral, estes problemas são contornados através do paradigma bayesiano, que apesar de disponibilizar metodologias computacionais robustas, são mais custosos comparados aos procedimentos inferenciais frequentistas.

Entre as sugestões para futuras pesquisas, consideram-se alguns aspectos na descrição: (i) da resposta longitudinal, como a modelagem do parâmetro de mistura do modelo inflacionado ou o uso de distribuições robustas para as respostas limitadas (Di Brisco et al., 2020; Bayes et al., 2012; Migliorati et al., 2019, 2018), para acomodar as medições atípicas de escores fornecidas pelos pacientes; e (ii) dos tempos de sobrevivência, através especificações mais flexíveis para a função de risco basal (Whittemore e Keller, 1986; Herndon e Harrell Jr, 1990; Rosenberg, 1995).

Embora os modelos conjuntos constituam uma importante ferramenta na análise de dados longitudinais e de sobrevivência, as limitações computacionais de abordagens frequentistas e bayesianas impedem a sua consolidação na Estatística Aplicada, sobretudo em problemas de maior complexidade. Neste sentido, a proposta de novos fatores para as correções de condicionamento e o desenvolvimento de procedimentos robustos e eficientes para a estimação são extensões almejadas deste trabalho. 


\section{Apêndice A}

\section{Conceitos gerais de análise numérica}

\section{A.1 Regras de quadratura para integração numérica}

Muitas aplicações requerem a avaliação de integrais de uma ou mais dimensões. Este procedimento, no entanto, pode ser bastante complexo, uma vez que algumas funções não possuem uma forma analítica para se encontrar as suas primitivas. Nestes casos, é necessário recorrer às aproximações numéricas. Uma maneira intuitiva de se aproximar uma integral é através de um somatório discreto e com pesos da função integrada, avaliada em pontos do domínio estrategicamente escolhidos. No caso univariado tem-se a seguinte formulação,

$$
\int_{\mathbb{R}} f(x) \approx \sum_{i=1}^{k} w_{i} \cdot f\left(x_{i}\right),
$$

em que $w_{i}$ são os pesos, e os pontos $\left\{x_{1}, \ldots, x_{k}\right\}$, que discretizam o domínio, o particionando em um número finito de subintervalos, são chamados de nós. Estes elementos, associados ao problema de integração numérica, constituem uma regra de quadratura e podem ser obtidos de variadas formas, a depender do integrando.

As regras de quadratura gaussiana, por exemplo, são construídas adequadamente para que a aproximação (A.1) se torne exata quando a função integrada for um polinômio de grau $2 n-1$. Em particular, a quadratura de Gauss-Hermite considera o problema de aproximar integrais do tipo

$$
\int_{\mathbb{R}} e^{-x^{2}} g(x) d x
$$

pelo seguinte somatório, 


$$
\sum_{i=1}^{k} w_{i} \cdot g\left(x_{i}\right)
$$

em que $g(\cdot)$ é uma função regular. Os nós e os pesos são determinados de forma que, se a função $g(\cdot)$ for um polinômio de grau máximo $2 k-1$, a aproximação (A.3) se torna exata. Assim, a construção desta regra de quadratura fornece os valores $x_{i}$, obtidos como a i-ésima raiz do polinômio de Hermite de grau $k$,

$$
H_{k}(x)=(-1)^{k} \cdot \exp \left(\frac{x^{2}}{2}\right) \cdot \frac{d^{k}}{d x^{k}}\left[\exp \left(\frac{-x^{2}}{2}\right)\right]
$$

e os pesos $w_{i}$ obtidos como

$$
w_{i}\left(x_{i}\right)=\frac{2^{k-1} k ! \sqrt{\pi}}{k^{2}\left(H_{k-1}\left(x_{i}\right)\right)}, \quad i=1, \ldots, k
$$

No caso multidimensional, a quadratura de Gauss Hermite considera a integração de funções $f$ : $\mathbb{R}^{m} \rightarrow \mathbb{R}$ da forma

$$
f(\boldsymbol{x})=w(\boldsymbol{x}) \cdot g(\boldsymbol{x})
$$

com $\boldsymbol{x}=\left(x_{1}, \ldots, x_{m}\right)^{\top}$ e $w(\boldsymbol{x})=\exp \left(-x_{1}^{2}\right) \cdot \ldots \cdot \exp \left(-x_{m}^{2}\right)$. Assim, a aproximação da integral avaliada é dada por,

$$
\int_{\mathbb{R}^{m}} f(\boldsymbol{x}) d x \approx \sum_{i_{1}=1}^{k_{1}} w_{i_{1}}^{(1)} \cdot \ldots \cdot \sum_{i_{m}=1}^{k_{m}} w_{i_{m}}^{(m)} \cdot g\left(x_{i_{1}}^{(1)}, \ldots, x_{i_{m}}^{(m)}\right),
$$

em que $x_{i_{r}}^{(r)}$ denota o $i_{r}$-ésimo zero do polinômio de Hermite, referente à $r$-ésima ordenada, e $w_{i_{m}}^{(r)}$ é o peso atribuído ao nó $x_{i_{r}}^{(r)}$.

\section{A.2 Método das diferenças finitas}

A habilidade de se expandir funções suaves em série de Taylor permite aproximar os valores das derivadas. No caso univariado, a expansão de segunda ordem de uma função $f(\cdot)$ de classe $\mathcal{C}^{2}$, pode ser rearranjada na forma

$$
\frac{f(x+h)-f(x)}{h}=f^{\prime}(x)+\frac{1}{2} h f^{\prime \prime}\left(x+c_{1} h\right),
$$

$c_{1} \in[0,1]$. A relação (A.7) pode ser escrita como

$$
\frac{f(x+h)-f(x)}{h}=f^{\prime}(x)+O(h) .
$$


O erro na aproximação de $f^{\prime}(x)$ à esquerda da equação (A.7) é devido ao negligenciamento do termo $\frac{1}{2} h f^{\prime \prime}(x+c h)$, que constitui um erro de truncamento. A fórmula (A.8) é conhecida como diferença finita progressiva. De forma similar, a expansão de $f(\cdot)$ pode ser expandida em torno do ponto $x-h$, obtendo-se

$$
\frac{f(x-h)-f(x)}{h}=f^{\prime}(x)+\frac{1}{2} h f^{\prime \prime}\left(x+c_{2} h\right),
$$

$c_{2} \in[0,1]$. A relação (A.9) pode ser escrita como

$$
\frac{f(x-h)-f(x)}{h}=f^{\prime}(x)+O(h) .
$$

A expansão (A.10) resulta na diferença finita regressiva. Se as expansões (A.7) e (A.9) são truncadas no elemento de terceira ordem, tem-se que

$$
f(x+h)=f(x)+h f^{\prime}(x)+\frac{1}{2} h f^{\prime \prime}(x)+O\left(h^{3}\right),
$$

e

$$
f(x-h)=f(x)-h f^{\prime}(x)+\frac{1}{2} h f^{\prime \prime}(x)+O\left(h^{3}\right) .
$$

Subtraindo a expressão (A.11) de (A.12), e dividindo por $h$, tem-se que

$$
\frac{f(x+h)-f(x-h)}{2 h}=f^{\prime}(x)+O\left(h^{2}\right) .
$$

A expressão (A.13) é chamada de diferença finita central. 


\section{Apêndice B}

\section{Variáveis basais do estudo do ICESP}

Neste capítulo encontram-se as variáveis utilizadas para a análise dos dados do ICESP. Na Tabela B.1 estão disponíveis as suas categorizações e distribuição na amostra dos 803 participantes do estudo.

Tabela B.1: Descrição das características basais dos 803 participantes do estudo do ICESP.

\begin{tabular}{|c|c|c|c|}
\hline Variável & Categorias & Codificação & $n(\%)$ \\
\hline \multirow{3}{*}{ Status do Câncer } & Controlado / Remissão & Status-CR & $47(6,0)$ \\
\hline & Ativo / Diagnóstico Recente & Status-AD & $400(50,7)$ \\
\hline & Recaída / Progressão & Status-RP & $342(43,3)$ \\
\hline \multirow{3}{*}{ Extensão do Câncer } & Limitado & ExtCancer-L & $261(33,1)$ \\
\hline & Localmente avançado & ExtCancer-LA & $279(35,4)$ \\
\hline & Metástases à distância / Leucemias & ExtCancer-ML & $249(31,5)$ \\
\hline \multirow{2}{*}{ Tipo Admissão na UTI } & Planejada & TipoAdm-P & $445(43,6)$ \\
\hline & Não planejada & TipoAdm-N & $344(53,4)$ \\
\hline \multirow[b]{2}{*}{ Insuficiência cardíaca } & Classe funcional NYHA III e IV & Insuf-S & $33(4,2)$ \\
\hline & Não & Insuf-N & $756(95,8)$ \\
\hline \multirow[b]{2}{*}{ Radioterapia prévia } & $\operatorname{Sim}$ & Radio-S & $172(21,8)$ \\
\hline & Não & Radio-N & $617(78,2)$ \\
\hline \multirow[b]{2}{*}{ Quimioterapia prévia } & $\operatorname{Sim}$ & Quimio-S & $301(38,2)$ \\
\hline & Não & Qumio-N & $488(61,8)$ \\
\hline \multirow{5}{*}{ ECOG } & 0 - Totalmente ativo & ECOG-0 & $319(40,5)$ \\
\hline & 1 - Restrito a atividades fisicamente extenuantes & ECOG-1 & $217(27,5)$ \\
\hline & 2 - Capaz de autocuidado, mas incapaz de realizar atividades de trabalho & ECOG-2 & $116(14,7)$ \\
\hline & 3 - Capaz de autocuidade limitado & ECOG-3 & $97(12,3)$ \\
\hline & 4 - Confinado à cama/ cadeira de rodas por mais de 50\% do tempo & ECOG-4 & $40(5,0)$ \\
\hline \multirow[b]{2}{*}{ Diabetes } & Diabetes mellitus/ dependente de insulina & Diab-S & $123(15,6)$ \\
\hline & Não & Diab-N & $666(84,4)$ \\
\hline \multirow{3}{*}{ Tabagismo } & Atual & Tab-A & $108(13,8)$ \\
\hline & Prévio & Tab-P & $376(47,6)$ \\
\hline & Não & $\mathrm{Tab}-\mathrm{N}$ & $305(38,6)$ \\
\hline \multirow[b]{2}{*}{ Alcoolismo } & $\operatorname{Sim}$ & Alc-S & $89(11,3)$ \\
\hline & Não & $A l c-N$ & $700(88,7)$ \\
\hline \multirow[b]{2}{*}{ Cirurgia } & $\operatorname{Sim}$ & Cirurgia-S & $476(60,3)$ \\
\hline & Não & Cirurgia-N & $313(39,7)$ \\
\hline \multirow{3}{*}{ Ventilação } & Espontânea & Ventilacao-E & $603(76,4)$ \\
\hline & Mecânica invasiva & Ventilacao-M & $155(19,4)$ \\
\hline & Não invasiva & Ventilacao-N & $31(4,0)$ \\
\hline
\end{tabular}




\section{Apêndice C}

\section{Códigos}

As rotinas a seguir são extrações de códigos em linguagem R (R Core Team, 2020) utilizadas para a implementação dos modelos conjuntos estudados neste trabalho. Todas elas são adaptações de funções disponíveis no pacote JM (Rizopoulos, 2010) para acomodar componentes longitudinais com distribuição beta e beta inflacionada em um. Na Seção C.1 encontram-se as estruturas de dados usadas para implementar a verossimilhança marginal dos modelos conjuntos de maneira vetorizada. Na Seção C.2 a verossimilhança do modelo BEI-WEI é disponibilizada para ilustrar a implementação do submodelo longitudinal para respostas limitadas na abordagem conjunta. E na Seção C.3 mostra-se como a metodologia proposta para o condicionamento da estimação pode ser aplicada no algoritmo BFGS através da função optim.

\section{C.1 Estruturas de dados usadas para a vetorização no $\mathbf{R}$}

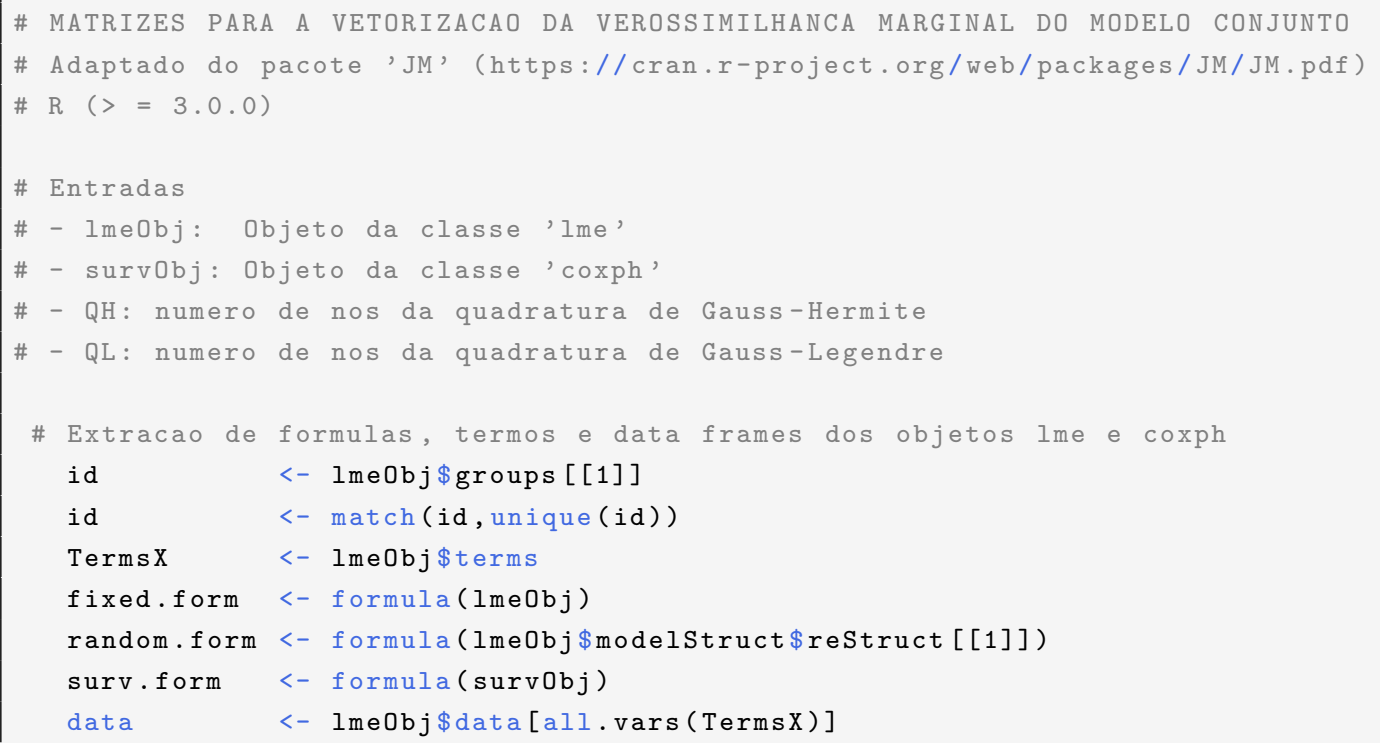




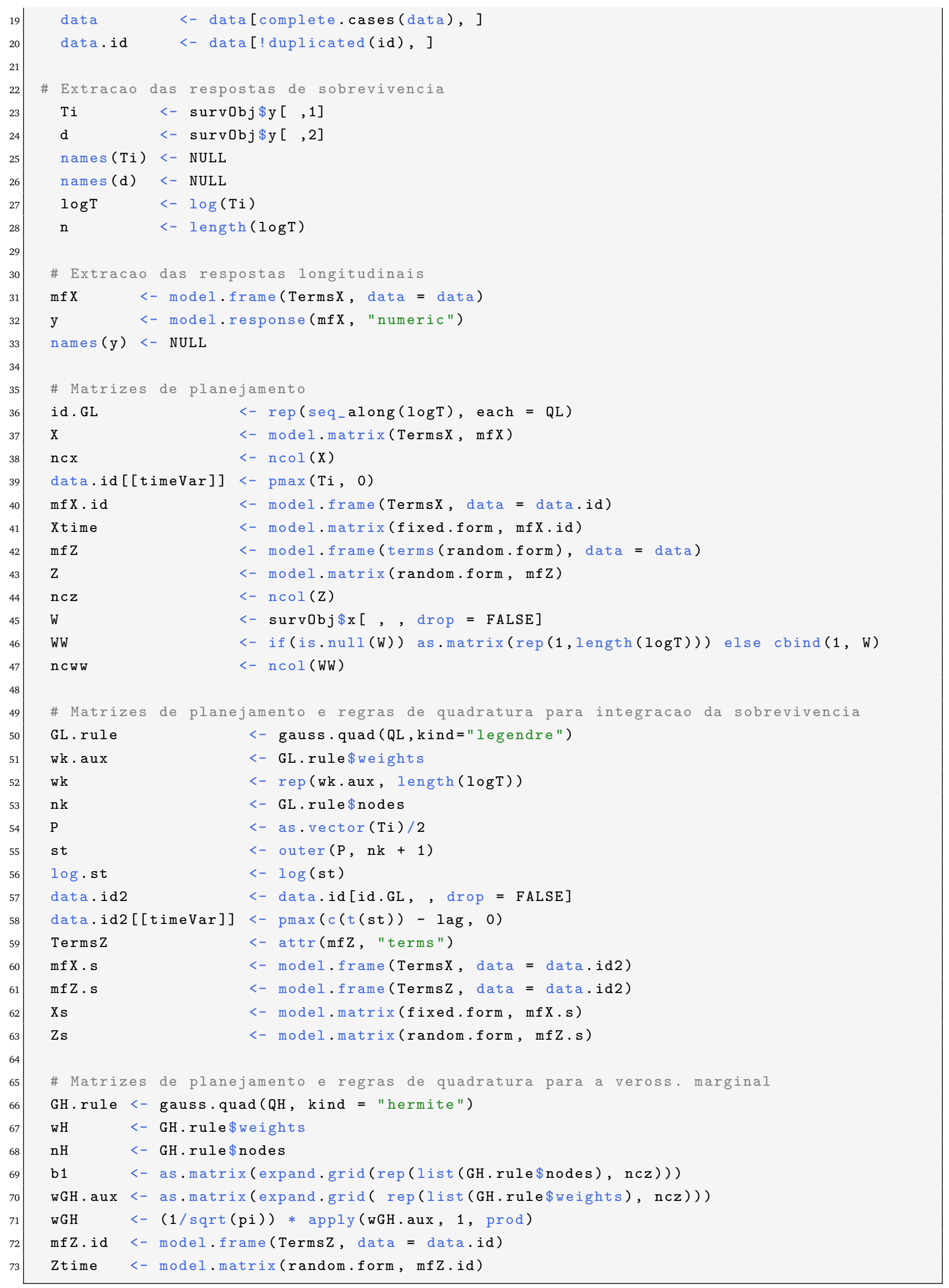




\section{C.2 Verossimilhança marginal do modelo BE-WEI}

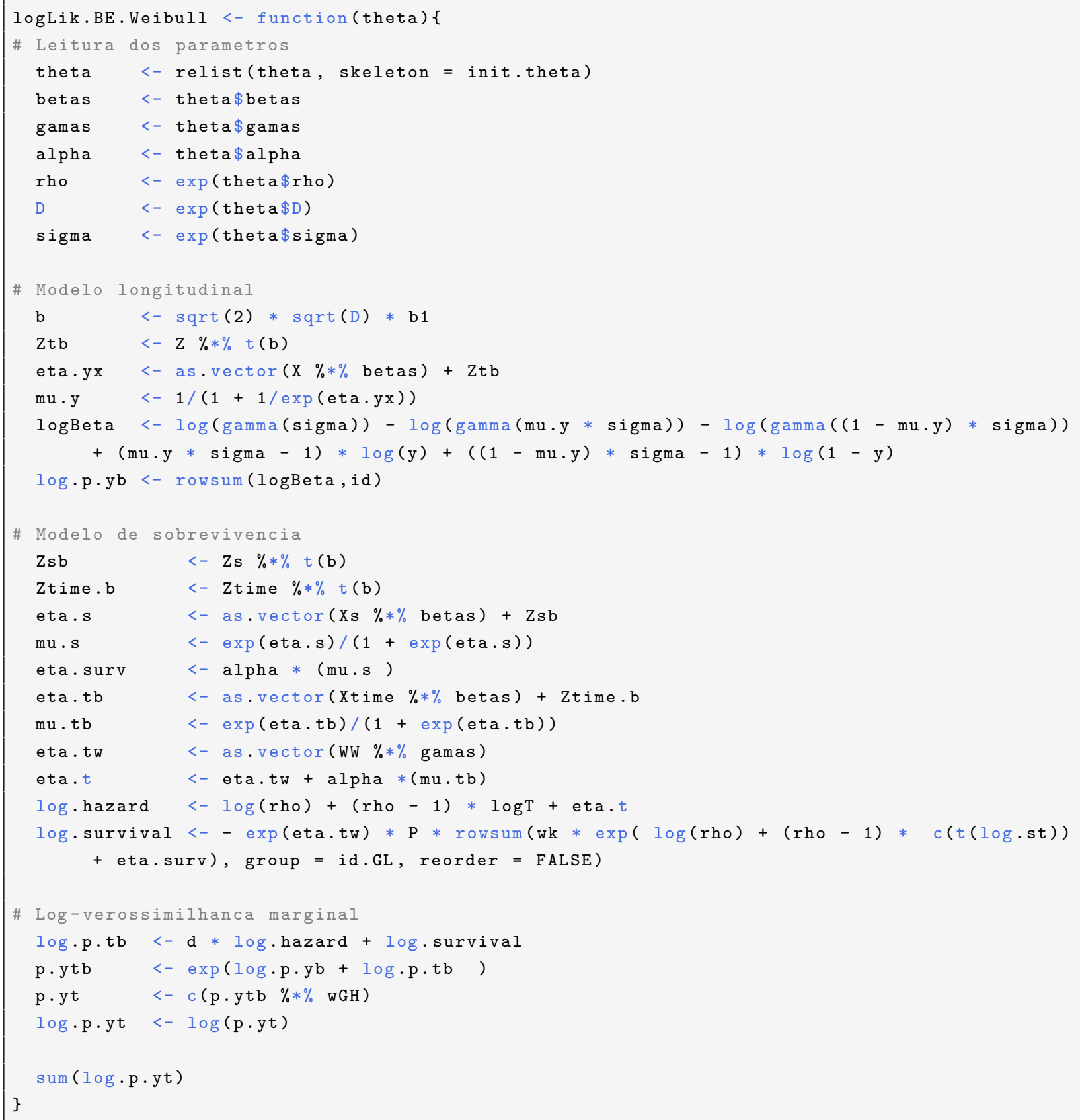




\section{C.3 Reescala dos parâmetros através do optim}

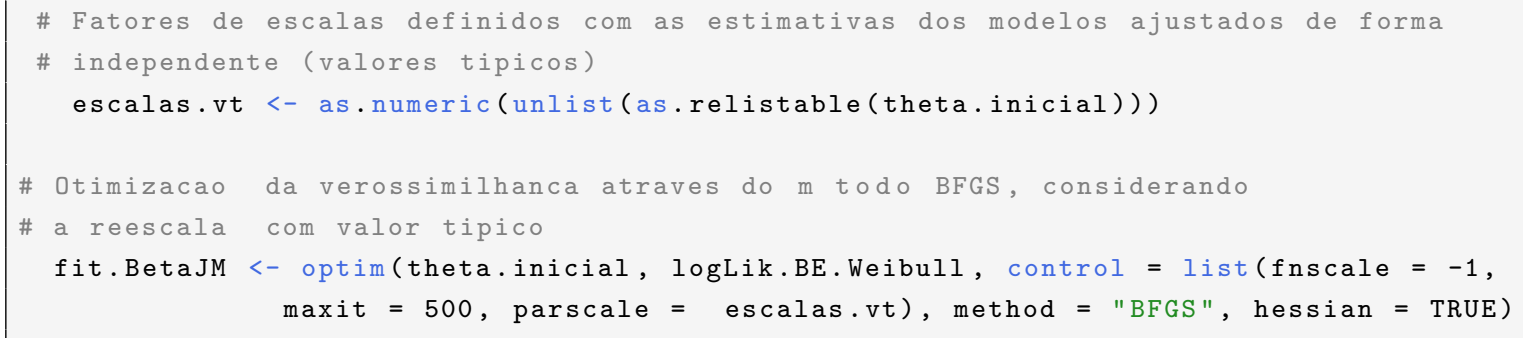




\section{Referências Bibliográficas}

Alvares et al.(2020) Danilo Alvares, Carmen Armero, Anabel Forte e Nicolas Chopin. Sequential monte carlo methods in bayesian joint models for longitudinal and time-to-event data. Statistical Modelling, página 1471082X20916088. Citado na pág. 39

Atkinson(1985) Anthony Curtes Atkinson. Plots, transformations and regression; an introduction to graphical methods of diagnostic regression analysis. Relatório técnico. Citado na pág. 8

Barndorff-Nielsen e Jørgensen(1991) Ole E Barndorff-Nielsen e Bent Jørgensen. Some parametric models on the simplex. Journal of multivariate analysis, 39(1):106-116. Citado na pág. 2, 7

Bayes et al.(2012) Cristian L Bayes, Jorge L Bazán, Catalina García et al. A new robust regression model for proportions. Bayesian Analysis, 7(4):841-866. Citado na pág. 96

Beiranvand et al.(2017) Vahid Beiranvand, Warren Hare e Yves Lucet. Best practices for comparing optimization algorithms. Optimization and Engineering, 18(4):815-848. Citado na pág. 65

Bender et al.(2005) Ralf Bender, Thomas Augustin e Maria Blettner. Generating survival times to simulate cox proportional hazards models. Statistics in medicine, 24(11):1713-1723. Citado na pág. 59, 60, 61

Billingham e Abrams(2002) LJ Billingham e KR Abrams. Simultaneous analysis of quality of life and survival data. Statistical Methods in Medical Research, 11(1):25-48. Citado na pág. 1

Booth et al.(2001) James G Booth, James P Hobert e Wolfgang Jank. A survey of monte carlo algorithms for maximizing the likelihood of a two-stage hierarchical model. Statistical Modelling, 1(4):333-349. Citado na pág. 46

Boyles(1983) Russell A Boyles. On the convergence of the em algorithm. Journal of the Royal Statistical Society: Series B (Methodological), 45(1):47-50. Citado na pág. 44 
Brent(2013) Richard P Brent. Algorithms for minimization without derivatives. Courier Corporation. Citado na pág. 61

Cao et al.(2005) B Cao, W Jank e G Jones. Ascent-based monte carlo em. Journal of the Royal Statistical Society: Series B, 67:235-252. Citado na pág. 46

Cao et al. (2018) Yuting Cao, Yi Jiang, Xiao Lin, Jinsong Liu, Tao Lu, Wenjun Cheng e Fangrong Yan. Dynamic prediction of outcome for patients with ovarian cancer: application of a joint model for longitudinal cancer antigen 125 values. International Journal of Gynecologic Cancer, 28(1). Citado na pág. 18

Chan e Ledolter(1995) KS Chan e Johannes Ledolter. Monte carlo em estimation for time series models involving counts. Journal of the American Statistical Association, 90(429):242-252. Citado na pág. 46

Cox(1972) David R Cox. Regression models and life-tables. Journal of the Royal Statistical Society: Series B (Methodological), 34(2):187-202. Citado na pág. 20

Crowther e Lambert(2013) Michael J Crowther e Paul C Lambert. Simulating biologically plausible complex survival data. Statistics in medicine, 32(23):4118-4134. Citado na pág. viii, x, 59, 60, 61, 62

Dafni e Tsiatis(1998) Urania G Dafni e Anastasios A Tsiatis. Evaluating surrogate markers of clinical outcome when measured with error. Biometrics, páginas 1445-1462. Citado na pág. 22

Dempster et al.(1977) Arthur P Dempster, Nan M Laird e Donald B Rubin. Maximum likelihood from incomplete data via the em algorithm. Journal of the Royal Statistical Society: Series B (Methodological), 39(1):1-22. Citado na pág. 31, 42, 44

Di Brisco et al.(2020) Agnese Maria Di Brisco, Sonia Migliorati e Andrea Ongaro. Robustness against outliers: A new variance inflated regression model for proportions. Statistical Modelling, 20(3):274309. Citado na pág. 96

Efron(1979) Bradley Efron. Bootstrap methods: another look at the jackknife. The Annals of Statistics, 7 (1):1-26. Citado na pág. 34

Ferrari e Cribari-Neto(2004) Silvia Ferrari e Francisco Cribari-Neto. Beta regression for modelling rates and proportions. Journal of applied statistics, 31(7):799-815. Citado na pág. 2, 4, 10, 27

Fitzmaurice et al.(2008) Garrett Fitzmaurice, Marie Davidian, Geert Verbeke e Geert Molenberghs. Longitudinal data analysis. CRC press. Citado na pág. 17 
Galvis et al.(2014) Diana M Galvis, Dipankar Bandyopadhyay e Victor H Lachos. Augmented mixed beta regression models for periodontal proportion data. Statistics in medicine, 33(21):3759-3771. Citado na pág. $2,7,10,28$

Gill et al.(2019) Philip E Gill, Walter Murray e Margaret H Wright. Practical optimization. SIAM. Citado na pág. $48,50,54,58,63,78$

Gleason(1988) John R Gleason. Algorithms for balanced bootstrap simulations. The American Statistician, 42(4):263-266. Citado na pág. 35

Gould et al.(2015) A Gould, Mark Ernest Boye, Michael J Crowther, Joseph G Ibrahim, George Quartey, Sandrine Micallef e Frederic Y Bois. Joint modeling of survival and longitudinal non-survival data: current methods and issues. report of the dia bayesian joint modeling working group. Statistics in medicine, 34(14):2181-2195. Citado na pág. 18

Group(1990) The EuroQol Group. Euroqol-a new facility for the measurement of health-related quality of life. Health policy, 16(3):199-208. Citado na pág. 6

Gupta e Chen(2011) Maya R Gupta e Yihua Chen. Theory and use of the EM algorithm. Now Publishers Inc. Citado na pág. 44

Hahn(2008) Eugene David Hahn. Mixture densities for project management activity times: A robust approach to pert. European Journal of operational research, 188(2):450-459. Citado na pág. 7

Hatfield et al.(2012) Laura A Hatfield, Mark E Boye, Michelle D Hackshaw e Bradley P Carlin. Multilevel bayesian models for survival times and longitudinal patient-reported outcomes with many zeros. Journal of the American Statistical Association, 107(499):875-885. Citado na pág. 2

Henderson et al.(2002) Robin Henderson, Peter Diggle e Angela Dobson. Identification and efficacy of longitudinal markers for survival. Biostatistics, 3(1):33-50. Citado na pág. 83

Herndon e Harrell Jr(1990) James E Herndon e Frank E Harrell Jr. The restricted cubic spline hazard model. Communications in Statistics-Theory and Methods, 19(2):639-663. Citado na pág. 20, 96

Higham(2002) Nicholas J Higham. Accuracy and stability of numerical algorithms. SIAM. Citado na pág. 50

Hsieh et al.(2006) Fushing Hsieh, Yi-Kuan Tseng e Jane-Ling Wang. Joint modeling of survival and longitudinal data: likelihood approach revisited. Biometrics, 62(4):1037-1043. Citado na pág. 20, 78 
Hunger et al.(2011) Matthias Hunger, Jens Baumert e Rolf Holle. Analysis of sf-6d index data: is beta regression appropriate? Value in Health, 14(5):759-767. Citado na pág. 2, 28

Hunger et al.(2012) Matthias Hunger, Angela Döring e Rolf Holle. Longitudinal beta regression models for analyzing health-related quality of life scores over time. BMC medical research methodology, 12(1): 144. Citado na pág. 7,9

Ibrahim et al.(2004) Joseph G Ibrahim, Ming-Hui Chen e Debajyoti Sinha. Bayesian methods for joint modeling of longitudinal and survival data with applications to cancer vaccine trials. Statistica Sinica, páginas $863-883$. Citado na pág. 18

Ibrahim et al.(2010) Joseph G Ibrahim, Haitao Chu e Liddy M Chen. Basic concepts and methods for joint models of longitudinal and survival data. Journal of Clinical Oncology, 28(16):2796. Citado na pág. 18

Kalbfleisch e Prentice(2011) John D Kalbfleisch e Ross L Prentice. The statistical analysis of failure time data, volume 360. John Wiley \& Sons. Citado na pág. 16, 17, 19

Kaplan e Meier(1958) Edward L Kaplan e Paul Meier. Nonparametric estimation from incomplete observations. Journal of the American statistical association, 53(282):457-481. Citado na pág. 12

Kieschnick e McCullough(2003) Robert Kieschnick e Bruce D McCullough. Regression analysis of variates observed on $(0,1)$ : percentages, proportions and fractions. Statistical modelling, 3(3):193-213. Citado na pág. 7, 27

Klein e Moeschberger(2003) John P Klein e Melvin L Moeschberger. Survival analysis: techniques for censored and truncated data, volume 1230. Springer. Citado na pág. 78

Król et al.(2016) Agnieszka Król, Loïc Ferrer, Jean-Pierre Pignon, Cécile Proust-Lima, Michel Ducreux, Olivier Bouché, Stefan Michiels e Virginie Rondeau. Joint model for left-censored longitudinal data, recurrent events and terminal event: Predictive abilities of tumor burden for cancer evolution with application to the ffcd 2000-05 trial. Biometrics, 72(3):907-916. Citado na pág. 18

Kumaraswamy(1980) Ponnambalam Kumaraswamy. A generalized probability density function for double-bounded random processes. Journal of hydrology, 46(1-2):79-88. Citado na pág. 7 
Kypriotakis et al.(2016) George Kypriotakis, Damon J Vidrine, Linda E Francis e Julia H Rose. The longitudinal relationship between quality of life and survival in advanced stage cancer. Psycho-Oncology, 25(2):225-231. Citado na pág. 3

Li e Luo(2017) Kan Li e Sheng Luo. Functional joint model for longitudinal and time-to-event data: an application to alzheimer's disease. Statistics in medicine, 36(22):3560-3572. Citado na pág. 18

Lin et al.(2002) Haiqun Lin, Charles E McCulloch e Susan T Mayne. Maximum likelihood estimation in the joint analysis of time-to-event and multiple longitudinal variables. Statistics in Medicine, 21(16): 2369-2382. Citado na pág. 38

Liu e Li(2016) Fang Liu e Qing Li. A bayesian model for joint analysis of multivariate repeated measures and time to event data in crossover trials. Statistical methods in medical research, 25(5):2180-2192. Citado na pág. 2

Lundberg et al.(1999) Lena Lundberg, Magnus Johannesson, Dag GL Isacson e Lars Borgquist. Healthstate utilities in a general population in relation to age, gender and socioeconomic factors. The European Journal of Public Health, 9(3):211-217. Citado na pág. 6

Magalhães(2006) Marcos Nascimento Magalhães. Probabilidade e variáveis aleatórias. Edusp. Citado na pág. 60

Martins et al.(2016) Rui Martins, Giovani L Silva e Valeska Andreozzi. Bayesian joint modeling of longitudinal and spatial survival aids data. Statistics in medicine, 35(19):3368-3384. Citado na pág. 18

Migliorati et al.(2018) Sonia Migliorati, Agnese Maria Di Brisco, Andrea Ongaro et al. A new regression model for bounded responses. Bayesian Analysis, 13(3):845-872. Citado na pág. 96

Migliorati et al.(2019) Sonia Migliorati, Agnese M Di Brisco e Andrea Ongaro. The flexible beta regression model. Data Analysis and Applications 1: Clustering and Regression, Modeling-estimating, Forecasting and Data Mining, 2:39-52. Citado na pág. 96

Molenberghs et al.(2014) Geert Molenberghs, Garrett Fitzmaurice, Michael G Kenward, Anastasios Tsiatis e Geert Verbeke. Handbook of missing data methodology. CRC Press. Citado na pág. 11, 16, 17, 29

Nash(2014) John C Nash. Nonlinear parameter optimization using $R$ tools. John Wiley \& Sons. Citado na pág. 54 
Neath et al.(2013) Ronald C Neath et al. On convergence properties of the monte carlo em algorithm. Em Advances in modern statistical theory and applications: a Festschrift in Honor of Morris L. Eaton, páginas 43-62. Institute of Mathematical Statistics. Citado na pág. 44, 45

Nocedal e Wright(2006) Jorge Nocedal e Stephen Wright. Numerical optimization. Springer Science \& Business Media. Citado na pág. 39, 48, 50, 54, 56, 63, 78

Normilio-Silva et al.(2016) Karina Normilio-Silva, Adelaide Cristina de Figueiredo, Antonio Carlos Pedroso-de Lima, Gisela Tunes-da Silva, Adriana Nunes da Silva, Andresa Delgado Dias Levites, Ana Tereza de Simone, Patrícia Lopes Safra, Roberta Zancani, Paula Camilla Tonini et al. Long-term survival, quality of life, and quality-adjusted survival in critically ill patients with cancer. Critical care medicine, 44(7):1327-1337. Citado na pág. 6, 77, 84

Ospina e Ferrari(2012) Raydonal Ospina e Silvia LP Ferrari. A general class of zero-or-one inflated beta regression models. Computational Statistics \& Data Analysis, 56(6):1609-1623. Citado na pág. 2, 4, 28

Öztürk e Akdeniz(2000) Fikri Öztürk e Fikri Akdeniz. Ill-conditioning and multicollinearity. Linear Algebra and Its Applications, 321(1-3):295-305. Citado na pág. 51

Papageorgiou et al.(2019) Grigorios Papageorgiou, Katya Mauff, Anirudh Tomer e Dimitris Rizopoulos. An overview of joint modeling of time-to-event and longitudinal outcomes. Annual review of statistics and its application. Citado na pág. 18

Philipson et al.(2020) Pete Philipson, Graeme L Hickey, Michael J Crowther e Ruwanthi KolamunnageDona. Faster monte carlo estimation of joint models for time-to-event and multivariate longitudinal data. Computational Statistics \& Data Analysis, página 107010. Citado na pág. 38

Prentice(1982) Ross L Prentice. Covariate measurement errors and parameter estimation in a failure time regression model. Biometrika, 69(2):331-342. Citado na pág. 17

Proust-Lima et al.(2019) Cécile Proust-Lima, Viviane Philipps e Jean-François Dartigues. A joint model for multiple dynamic processes and clinical endpoints: Application to alzheimer's disease. Statistics in Medicine, 38(23):4702-4717. Citado na pág. 18

Quarteroni et al.(2010) Alfio Quarteroni, Riccardo Sacco e Fausto Saleri. Numerical mathematics, volume 37. Springer Science \& Business Media. Citado na pág. 31, 52, 61 
R Core Team(2020) R Core Team. R: A Language and Environment for Statistical Computing. R Foundation for Statistical Computing, Vienna, Austria, 2020. URL https://www.R-project.org/. Citado na pág. 63, 78, 101

Rabin et al.(2011) Rosalind Rabin, Mandy Oemar, Mark Oppe, Bas Janssen e Michael Herdman. Eq-5d-31 user guide: Basic information on how to use the eq-5d-31 instrument. Rotterdam: EuroQol Group, 22. Citado na pág. 6

Rizopoulos(2010) Dimitris Rizopoulos. Jm: An r package for the joint modelling of longitudinal and time-to-event data. Journal of Statistical Software (Online), 35(9):1-33. Citado na pág. 79, 101

Rizopoulos(2011) Dimitris Rizopoulos. Dynamic predictions and prospective accuracy in joint models for longitudinal and time-to-event data. Biometrics, 67(3):819-829. Citado na pág. 18, 23

Rizopoulos(2012) Dimitris Rizopoulos. Joint models for longitudinal and time-to-event data: With applications in $R$. CRC press. Citado na pág. 2, 3, 23, 38, 43, 46, 80

Rizopoulos et al.(2009) Dimitris Rizopoulos, Geert Verbeke e Emmanuel Lesaffre. Fully exponential laplace approximations for the joint modelling of survival and longitudinal data. Journal of the Royal Statistical Society: Series B (Statistical Methodology), 71(3):637-654. Citado na pág. 34

Rizopoulos et al.(2017) Dimitris Rizopoulos, Geert Molenberghs e Emmanuel MEH Lesaffre. Dynamic predictions with time-dependent covariates in survival analysis using joint modeling and landmarking. Biometrical Journal, 59(6):1261-1276. Citado na pág. 18

Rosenberg(1995) Philip S Rosenberg. Hazard function estimation using b-splines. Biometrics, páginas 874-887. Citado na pág. 20, 96

Rouanet et al.(2016) Anaïs Rouanet, Pierre Joly, Jean-François Dartigues, Cécile Proust-Lima e Hélène Jacqmin-Gadda. Joint latent class model for longitudinal data and interval-censored semi-competing events: Application to dementia. Biometrics, 72(4):1123-1135. Citado na pág. 18

Rue et al.(2017) Montserrat Rue, Eleni-Rosalina Andrinopoulou, Danilo Alvares, Carmen Armero, Anabel Forte e Lluis Blanch. Bayesian joint modeling of bivariate longitudinal and competing risks data: An application to study patient-ventilator asynchronies in critical care patients. Biometrical Journal, 59(6): 1184-1203. Citado na pág. 2 
Shrestha et al.(2019) Anne Shrestha, Charlene Martin, Maria Burton, Stephen Walters, Karen Collins e Lynda Wyld. Quality of life versus length of life considerations in cancer patients: a systematic literature review. Psycho-oncology, 28(7):1367-1380. Citado na pág. 1

Silva(2015) Karina Normilio da Silva. Qualidade de vida relacionada à saúde e tempo de sobrevida ajustado para a qualidade de vida em pacientes com câncer internados em unidades de terapia intensiva. Tese de Doutorado, Universidade de São Paulo. Citado na pág. 6

Smithson e Verkuilen(2006) Michael Smithson e Jay Verkuilen. A better lemon squeezer? maximumlikelihood regression with beta-distributed dependent variables. Psychological methods, 11(1):54. Citado na pág. viii, 8,9

Song et al.(2017) Hui Song, Yingwei Peng e Dongsheng Tu. Jointly modeling longitudinal proportional data and survival times with an application to the quality of life data in a breast cancer trial. Lifetime data analysis, 23(2):183-206. Citado na pág. 2

Soto et al.(2020) Diana Carolina Franco Soto, Antonio Carlos Pedroso de Lima e Julio da Motta Singer. Un modelo birnbaum-saunders para el análisis conjunto de datos de supervivencia y longitudinales de insuficiencia cardíaca congestiva/a birnbaum-saunders model for joint survival and longitudinal analysis of congestive heart failure data. Revista Colombiana de Estadistica, 43(1):83. Citado na pág. 18, 77

Sweeting e Thompson(2011) Michael J Sweeting e Simon G Thompson. Joint modelling of longitudinal and time-to-event data with application to predicting abdominal aortic aneurysm growth and rupture. Biometrical Journal, 53(5):750-763. Citado na pág. 22

Thanedar et al.(1990) PB Thanedar, JS Arora, GY Li e TC Lin. Robustness, generality and efficiency of optimization algorithms for practical applications. Structural optimization, 2(4):203-212. Citado na pág. 50

Trefethen e Bau III(1997) Lloyd N Trefethen e David Bau III. Numerical linear algebra, volume 50. Siam. Citado na pág. 56

Tsiatis e Davidian(2001) Anastasios A Tsiatis e Marie Davidian. A semiparametric estimator for the proportional hazards model with longitudinal covariates measured with error. Biometrika, 88(2):447458. Citado na pág. 22

Tsiatis e Davidian(2004) Anastasios A Tsiatis e Marie Davidian. Joint modeling of longitudinal and time-to-event data: an overview. Statistica Sinica, páginas 809-834. Citado na pág. 2, 18 
Verbeke e Davidian(2009) Geert Verbeke e M Davidian. Joint models for longitudinal data: Introduction and overview. Citado na pág. 17

Vesz et al.(2013) Patrini Silveira Vesz, Monise Costanzi, Débora Stolnik, Camila Dietrich, Karen Lisiane Chini de Freitas, Letícia Aparecida Silva, Carolina Schünke de Almeida, Camila Oliveira de Souza, Jorge Ondere, Dante Lucas Santos Souza et al. Functional and psychological features immediately after discharge from an intensive care unit: Prospective cohort study. Revista Brasileira de terapia intensiva, 25(3):218. Citado na pág. 1

Viviani et al.(2014) Sara Viviani, Marco Alfó e Dimitris Rizopoulos. Generalized linear mixed joint model for longitudinal and survival outcomes. Statistics and Computing, 24(3):417-427. Citado na pág. 18

Watkins(2004) David S Watkins. Fundamentals of matrix computations, volume 64. John Wiley \& Sons. Citado na pág. 52, 55, 58

Wei e Tanner(1990) Greg CG Wei e Martin A Tanner. A monte carlo implementation of the em algorithm and the poor man's data augmentation algorithms. Journal of the American statistical Association, 85 (411):699-704. Citado na pág. 45

Whittemore e Keller(1986) Alice S Whittemore e Joseph B Keller. Survival estimation using splines. Biometrics, páginas 495-506. Citado na pág. 20, 96

Wu(1983) CF Jeff Wu. On the convergence properties of the em algorithm. The Annals of statistics, páginas 95-103. Citado na pág. 44

Wu et al.(2019) Jing Wu, Ming-Hui Chen, Elizabeth D Schifano, Joseph G Ibrahim e Jeffrey D Fisher. A new bayesian joint model for longitudinal count data with many zeros, intermittent missingness, and dropout with applications to hiv prevention trials. Statistics in Medicine, 38(30):5565-5586. Citado na pág. 18

Wu et al.(2012) Lang Wu, Wei Liu, Grace Y Yi e Yangxin Huang. Analysis of longitudinal and survival data: joint modeling, inference methods, and issues. Journal of Probability and Statistics, 2012. Citado na pág. 77

Xu et al.(2014) Cong Xu, Paul D Baines e Jane-Ling Wang. Standard error estimation using the em algorithm for the joint modeling of survival and longitudinal data. Biostatistics, 15(4):731-744. Citado na pág. 44 
Yang et al.(2016) Lili Yang, Menggang Yu e Sujuan Gao. Joint models for multiple longitudinal processes and time-to-event outcome. Journal of statistical computation and simulation, 86(18):3682-3700. Citado na pág. 44

Yu et al.(2008) Menggang Yu, Jeremy M G Taylor e Howard M Sandler. Individual prediction in prostate cancer studies using a joint longitudinal survival-cure model. Journal of the American Statistical Association, 103(481):178-187. Citado na pág. 18

Zeng et al.(2005) Donglin Zeng, Jianwen Cai et al. Asymptotic results for maximum likelihood estimators in joint analysis of repeated measurements and survival time. The Annals of Statistics, 33(5):2132-2163. Citado na pág. 22 